
Albert-Ludwigs-Universität Freiburg i. Br.

Institut für Erziehungswissenschaften

Wissenschaftliche Arbeit

für die Zulassung zur ersten Staatsprüfung für das Lehramt

an Gymnasien

\title{
Sport in der Ganztagsschule
}

vorgelegt von:

\author{
Christopher Winterhalder \\ Cornelia-Schlosser-Allee 12
}

79111 Freiburg

Matrikelnummer: 2202546

eingereicht bei:

Prof. Dr. Matthias Nückles

Arbeitsbereich: Empirische Unterrichts- und Schulforschung 


\section{Abstract:}

PISA 2001 revealed that German students are missing several competencies in reading, writing and math. The students social background was one main reason for such inadequacies. The following questions arise: "Which actions should be initiated to prevent such outcomes?", "Do physical activations generally influence pupils learning development?", "Which benefits has sports in full day schools?"

Those are some of the questions which this paper tries to find answers to. 


\section{Inhaltsverzeichnis}

Seite

I. Inhaltsverzeichnis

II. Abbildungsverzeichnis

III

III. Tabellenverzeichnis IV

1. Einleitung

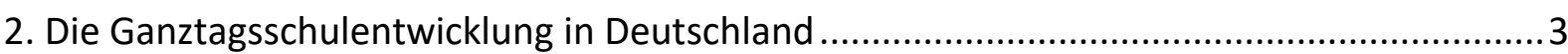

2.1 Bildungsstandards als Instrument der Schulentwicklung ......................................... 4

2.2 Pädagogische Leistungen von Ganztagsschulen ................................................ 6

2.3 Offene, gebundene und teilgebundene Ganztagsschulen ....................................... 7

2.4 Begründungen für die Einrichtung von Ganztagsschulen ......................................... 8

2.5 Schulkultur und die Bedeutung von außerschulischen Partnern................................. 8

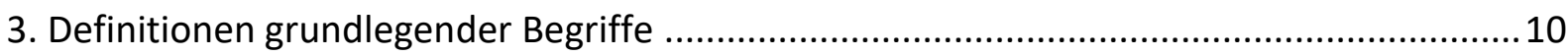

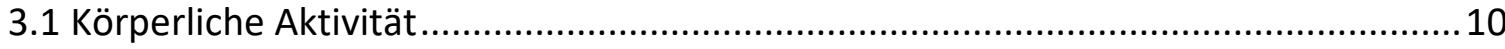

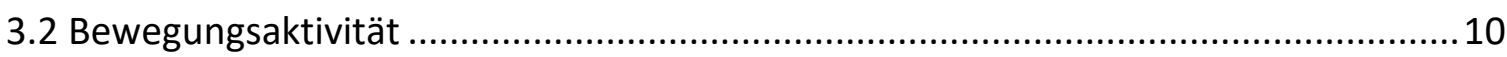

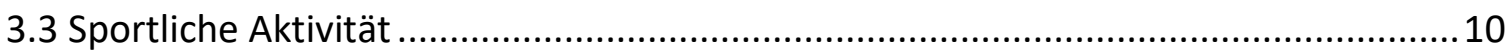

4. Sport, Spiel und Bewegung - Ein Grundbedürfnis Kinder und Jugendlicher

4.1 Bewegungsförderung und Entwicklungsförderung - Zwei untrennbare

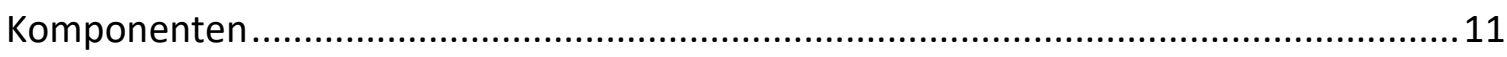

4.2 Individuelle Entwicklungsstufen Kinder und Jugendlicher....................................12

5. Zusammenhänge zwischen Bewegung und Gesundheit bei Kindern und Jugendlichen ......13

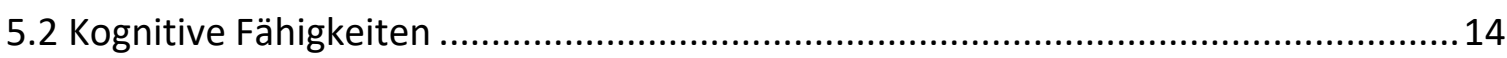

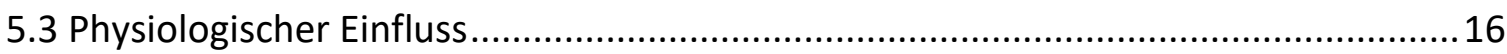

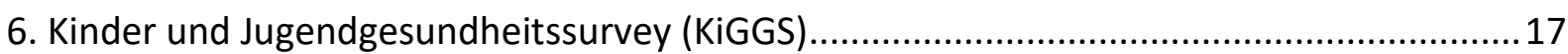

6.1 Ergebnisse der sportlichen Aktivität von Kindern zwischen 3 und 10 Jahren ............18

6.2 Ergebnisse der sportlichen Aktivität von Jugendlichen zwischen 11 und 17 Jahren .. 20

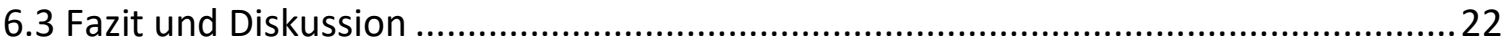

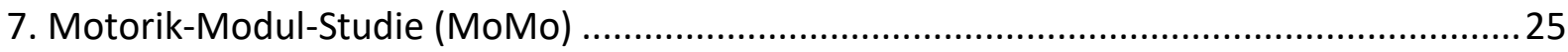

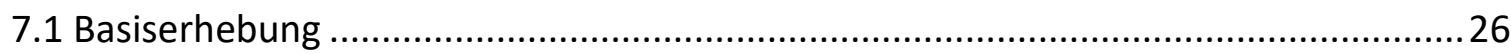

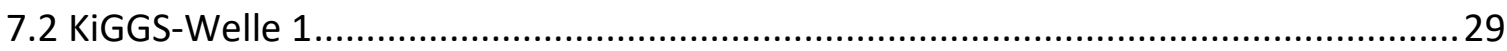

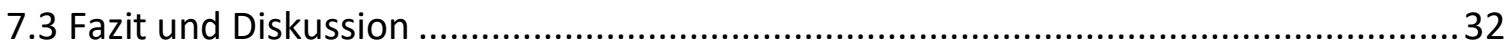


8. Praktische Umsetzungsmöglichkeiten des Sports in der Ganztagsschule ..........................34

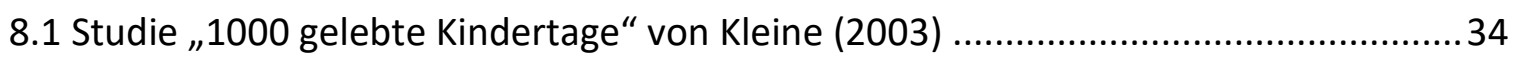

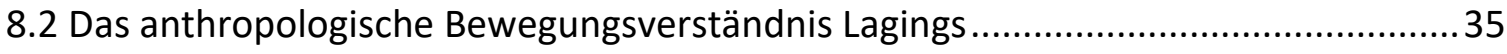

8.3 Kooperationen von Schule und Sport - ein Beitrag zur Schulkultur? ...........................36

8.4 Sport am Nachmittag - das additiv-duale Kooperationsmodell ..................................36

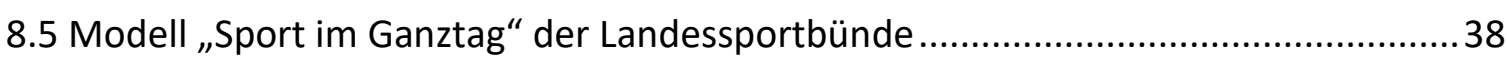

8.6 Das Modell der Bewegten Schule - Ein integrativer Ansatz ......................................40

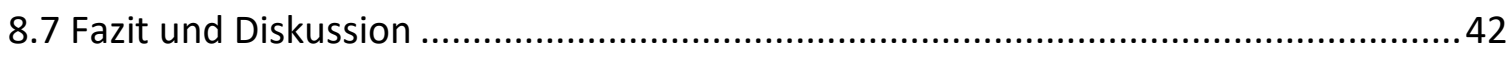

8.8 Interessenskonflikte der Kooperationspartner Schule und Sportverein ....................42

8.9 Das Basketball-Modell der Stadt Tübingen ............................................................ 45

9. Die acht Bausteine - Praktische Umsetzungsmöglichkeiten einer „Bewegten Schule“.......47

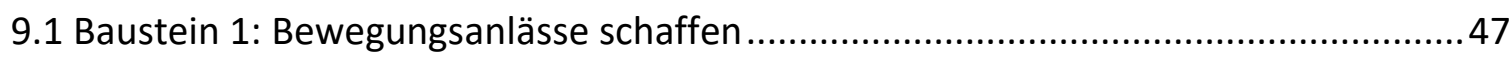

9.2 Baustein 2: Schulhof als Bewegungs- und Ruheraum ........................................... 48

9.3 Baustein 3: Bewegungsraum Schulgebäude ......................................................... 48

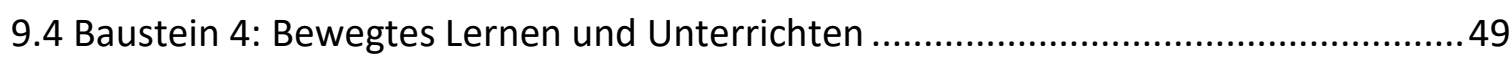

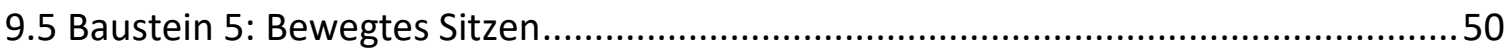

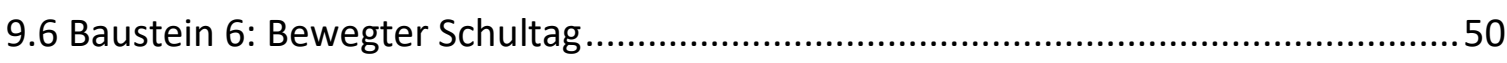

9.7 Baustein 7: Bewegungsangebote, Spielfeste und Öffnung von Schule ......................51

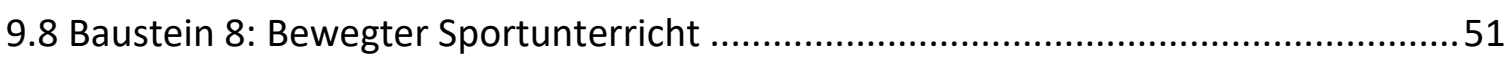

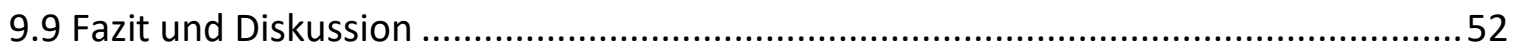

10. Bedeutung von Bewegung und Sport in informellen und nonformalen Settings im Kindes-

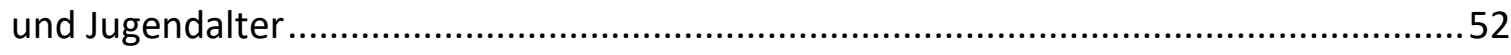

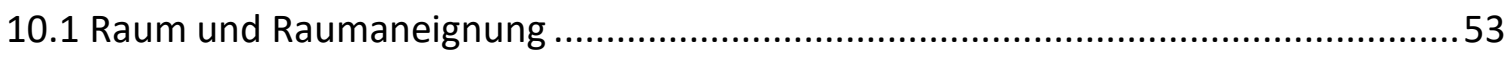

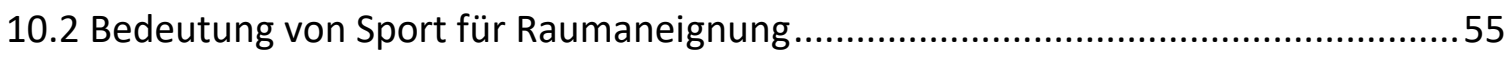

10.3 Einschränkungen in der Entwicklung des Städtebaus ...........................................56

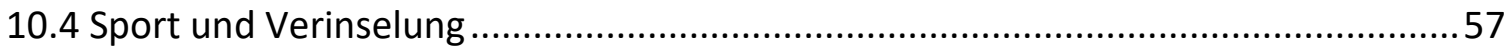

10.5 Unterschiede in der Raumaneignung von Jungen und Mädchen .............................59

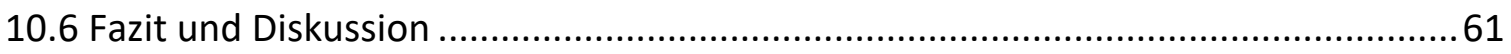

11. Die Ganztagsschule in Deutschland 2012/2013 .........................................................62

11.1 Entwicklungstendenzen der Ganztagsschulen in Deutschland ................................63

11.2 Ausbauperspektiven von Ganztagsschulen in Deutschland..................................65

12 Schlussbetrachtung .66

13. Literaturverzeichnis 72

14. Eidesstattliche Erklärung 


\section{Abbildungsverzeichnis}

Abb. 1: Anteil der 3- bis 10-jährigen Jungen und Mädchen, die weniger als einmal pro Woche Sport machen, nach Sozialstatus, Migrationshintergrund und Wohnregion (Quelle: Lampert et al., 2007, S. 638).

Abb. 2: Abnahme der körperlich-sportlichen Aktivität bei 11- bis 17-jährigen Jungen und Mädchen (Quelle: Lampert et al., 2007, S. 639)....

Abb. 3: Deutschlandkarte mit den insgesamt 167 Untersuchungsorten (Quelle: Opper et al., 2007, S. 885)

Abb. 4: Sport am Nachmittag - das additiv-duale Kooperationsmodell (Laging \& HildebrandtStramann, 2006, S. 16)

Abb. 5: Kooperation durch kompensatorische Ganztagsbildung - Modell „Sport im Ganztag“ (Laging \& Hildebrandt-Stramann, 2006, S. 17)

Abb. 6: Modell „Kooperation durch Inklusion von Bewegung, Spiel und Sport (Laging \& Hildebrandt-Stramann, 2006, S. 19)

Abb. 7: Mitwirkende des Basketball-Modells Tübingen (Fessler \& Ziroli, 1997, S. 148) 46

Abb. 8: Inselmodell nach Zeiher (Quelle: Deinet, 2005, S. 48). 57

Abb. 9: Anteil der Verwaltungseinheiten mit Ganztagsbetrieb an allen Verwaltungseinheiten - Schuljahre 2011/12 und 2012/13 (Primar- und Sekundarstufe I in privater und öffentlicher Trägerschaft (Quelle: Klemm, 2014, S. 13) 64

Abb. 10: Anteil der Schüler im Ganztagsschulbetrieb nach Angebotsform - Schuljahre 2011/12 und 2012/13 (Primar- und Sekundarstufe I in privater und öffentlicher Trägerschaft) (Quelle: Klemm, 2014, S. 15)

Abb. 11: Entwicklung der Ganztagsschulbeteiligung bis 2020/21 in Deutschland (Quelle:

Klemm, 2014, S. 20) . 66 


\section{Tabellenverzeichnis}

Tab. 1: Lebensspannenkonzept der menschlichen Motorik (Quelle: De Bock, 2012, S. 133) .13

Tab. 2: Erfassung der Motorik-Dimensionen im Kinder- und Jugendgesundheitssurvey (KiGGS) und Motorik-Modul (MoMo) (Quelle: Opper et al., 2007, S. 883).

Tab. 3: Verteilung von Körpermaßen, körperlich-sportlicher Aktivität und sozioökonomischem Status der gewichteten Motorik-Stichproben zur Basiserhebung und Welle 1 (Quelle: Albrecht et al., 2016, S. 298)

Tab. 4: Kohortenvergleich Motorikstichprobe; $n=2285$ zur Basiserhebung; $n=1441$ zur Welle 1; männliche Teilnehmer (Quelle: Albrecht et al., 2016, S. 300) 30

Tab. 5: Kohortenvergleich Motorikstichprobe; $n=2243$ zur Basiserhebung; $n=1379$ zur Welle 1; weibliche Teilnehmer (Quelle: Albrecht et al., 2016, S. 301) 


\section{Einleitung}

Sport, Spiel und Bewegung sind spätestens nach dem PISA-Schock von 2001 und den damit einhergehenden Reformen an der Gestaltung von Schule und insbesondere von Ganztagsschulen beteiligt. Die Ergebnisse der Studie „PISA 2000“ (vgl. Baumert et al., 2001) zeigten, dass fast ein Viertel der Jugendlichen die Schule mit äußerst dürftigen Kompetenzen in Lesen, Mathematik und Naturwissenschaften verlässt. Sowohl deren berufliche, als auch deren gesellschaftliche Integration sei demnach massiv gefährdet. Davon betroffen sind vor allem Kinder und Jugendliche aus einfachen sozialen Verhältnissen. Sowohl Einheimische also auch Migranten, die aus sozial schwachen Familien stammen, haben es laut PISA schwer, sich in den deutschen Schulen durchzusetzen. Baumert et al. (2001) beschreiben diesen Zusammenhang wie folgt: „Das deutsche Schulsystem ist Weltmeister in der sozialen Auslese, und es ist Spitzenreiter in der Produktion von Schulscheitern“ (zitiert nach Fessler \& Stibbe, 2007, S. 23).

Etliche Überlegungen nach neuen Maßnahmen, welche ein schlechtes Abschneiden deutscher Schülerinnen und Schüler ${ }^{1}$ zukünftig vermeiden sollen, wurden nach der PISA-Studie von 2001 eingeleitet. Initiativen wie bspw. die Einführung von Bildungsstandards, Lernstanderhebungen und zentrale Abschlussprüfungen wurden durchgesetzt. In diesem Zusammenhang wurde auch die Einführung von Ganztagsschulen diskutiert: „Die beiden zentralen Reformideen in der nationalen Reaktion auf PISA sind Ganztagsschulen und nationale Bildungsstandards", stellt Oelkers (2003, S. 36) fest.

Auch wenn das Fach Sport in den internationalen Leistungsvergleichsstudien keine Rolle spielte, hatten die dadurch ausgelösten bildungspolitischen Diskussionen Auswirkungen auf den Schulsport. Viele Maßnahmen wurden seitdem von den für die Schule zuständigen Landesministerien und der Kulturministerkonferenz durchgeführt. Dabei rückten die Bemühungen um einheitliche Bildungsstandards und der Ausbau der Ganztagsschule besonders in den Fokus. In diesen Zusammenhang ist auch der Sportunterricht in den Sog der PISA-Diskussion geraten. Der verpflichtende Sportunterricht wurde immens erweitert durch bspw. außerunterrichtliche Schulsportangebote. Diese werden entweder schulintern ermöglicht oder kommen in Kooperation mit außerschulischen Partnern wie Sportvereinen zustande (Laging, 2006). Neben den vielen Bewegungs- und Sportangeboten, die in der Schule angeboten

\footnotetext{
${ }^{1}$ Im Folgenden abgekürzt mit „SuS“.
} 
werden und wertvolle Erlebnisse der SuS mit sich bringen, kann der Sport auch andere erstklassige Fördermaßnahmen begünstigen. Zu nennen sind hierzu Angebote wie Psychomotorik oder Fitness sowie Angebote zur Gewaltprävention oder Sicherheit im Straßenverkehr (Laging, 2006; Grabs, 2004). Ein spannendes, praxisbezogenes Schulkonzept, das in diesem Zusammenhang genannt werden muss, ist das der "Bewegten Schule“. Sport und Bewegung sind in diesem Konzept zu einem eigenständigen Gestaltungselement geworden.

Doch warum ist Bewegung insbesondere für Kinder und Jugendliche überhaupt so wichtig? Es gibt unzählige Studien (vgl. u.a. Bös, 2003; Lampert et. al, 2007; Opper et. al, 2007), die einen erheblichen Rückgang des körperlichen und sportlichen Aktivitätsgrads von Kindern und Jugendlichen anmahnen. Auf der anderen Seite sieht Laging (2006) diese angebliche Problematik etwas differenzierter. Er vertritt die Meinung, dass man Kinder in der heutigen Zeit nicht per se als stets sitzend, fernsehsüchtig und fehlernährt verurteilen sollte. Laging (2006) nimmt entgegen der erwähnten Studienlage an, dass Bewegung für Kinder immer noch die wichtigste Aktivität überhaupt ist. Er nennt als unterstützenden Beweis seiner Aussagen die Studie „1000 gelebte Kindertage“ von Kleine (2003). Demnach waren Kinder an 98 \% der untersuchten 1000 Tage in Bewegung. Die Gesamtbewegungszeit über Mädchen und Jungen hinweg betrug durchschnittlich 3,5 Stunden pro Tag. Diese Zahlen widersprechen denjenigen Studien enorm, wonach Kinder sich angeblich bspw. nur 1 Stunde pro Tag bewegen (vgl. Bös, 2003). Folglich stehen sich diesbezüglich zwei konträre Ansichten gegenüber.

In dieser Abschlussarbeit wird die Thematik „Sport in der Ganztagsschule" aus mehreren Perspektiven durchleuchtet und diskutiert. Zunächst wird die Ganztagsschulentwicklung in Deutschland nach dem besagten PISA-Schock von 2001 sowie die unterschiedlichen Angebotstypen einer Ganztagsschule vorgestellt. Weiterhin soll die Frage beantwortet werden, was unter Bewegung sowie sportlicher und körperlicher Aktivität überhaupt zu verstehen ist. Darauf folgt eine Vorstellung der Studien „KiGGS“ (Lampert, Mensink, Rohman \& Woll, 2007) und „MoMo“ (Opper, Worth, Wagner et al., 2006), welche die Wichtigkeit von Sport für Kinder und Jugendliche nochmals unterstreichen. Zudem soll geklärt werden, welche SuS vom Ganztagsschulmodell besonders profitieren können.

Nach diesen medizinischen Grundlagen werden praktische Umsetzungsmöglichkeiten des Sports und Sportunterrichts innerhalb der Ganztagsschule genannt - u.a. das Konzept der „Bewegten Schule“ sowie verschiedene Kooperationsmodelle mit Vereinen. Darüber hinaus wird ebenfalls die Bedeutung von Bewegung und Sport in informellen und nonformalen Set- 
tings im Kindes- und Jugendalter erörtert sowie die notwendige Entwicklung und Neubildung von Räumen insbesondere in deutschen Städten kritisch reflektiert.

Zum Schluss sollen aktuelle Statistiken über die Verteilung von Ganztagsschulen in Deutschland die Basis für einen Ausblick und weitere Empfehlungsmöglichkeiten bilden: Wie kann eine Bewegte Schule der Zukunft aussehen und was muss im besten Fall noch am Modell der Ganztagsschule optimiert werden?

\section{Die Ganztagsschulentwicklung in Deutschland}

Schon vor über 45 Jahren wurden bildungspolitische und schulrelevante Diskussionen geführt, inwieweit der Schulalltag neu konzipiert und umgesetzt werden muss. Daraufhin folgten Ende der 60er Jahre die ersten Gesamtschulen - häufig in Ganztagesform. Die PISAErgebnisse von 2000 intensivierten die Einführung von Ganztagsschulen in Deutschland im Jahr 2003. Diese erste PISA-Untersuchung hat laut Laging (2014) „die seit langem bekannten kritischen Punkte des deutschen Schulwesens noch einmal vor Augen geführt" (S. 14). Er ist sich darüber hinaus sicher, dass diese „ersten Ergebnisse nicht nur [zeigen], dass die Schüler(innen) in Deutschland in den Kompetenzbereichen Lesen, Mathematik und Naturwissenschaften mittelmäßig im Vergleich der OECD-Staaten sind, sondern auch vor allem, dass die Leistungen innerhalb der deutschen Schülerschaft sehr weit streuen, in der Lesekompetenz sogar die größte aller Staaten aufweisen“ (ebd.).

Ein weitere Erkenntnis dieser Vergleichsstudie war, dass das Leistungsniveau erheblich mit den sozialen Lebensumständen der Kinder und Jugendlichen korrelierte (Baumert et al., 2001, S. 105; PISA Konsortium, 2004, S. 105; 2007, S. 239). Als Ursachenfeld für die schlechten Ergebnisse der SuS wurde ein unzureichend problemorientierter Unterricht verantwortlich gemacht, der eigeninitiiertes Lernen fördern sollte (Messner 2004). Hinzu kam auch der kritische Verweis auf den Halbtagsunterricht mit der viel zu frühen Schulselektion (Tillmann, 2005; Oelkers, 2003).

Daher wurden bei der Kultusministerkonferenz von 2002 „sieben Handlungsfelder aufgezeigt, die die zukünftige Schulentwicklung leiten sollte. Dazu gehören bspw. frühkindliche Bildung, die Kompetenzförderung in den grundlegenden Inhaltsbereichen, die Qualitätsverbesserung von Unterricht durch Standards und der, Ausbau schulischer und außerschulischer Ganztagsangebote““ (KMK, 2002; Laging 2014; zit. nach Tillmann, 2005, S. 52). 
Nach Holtappels (2005, S. 8 ff.) sollen Ganztagsschulen Verbesserungen:

- der "sozialen Infrastruktur" (Vereinbarkeit von Erwerbsarbeit und Familie durch Betreuung sowie Kompensation von Divergenzen im Anregungspotenzial der Wohnquartiere und der Teilhabe an kulturellen Angeboten),

- der „sozialerzieherischen Funktion“ (Stärkung der Erziehungsaufgabe als familienergänzende Maßnahme aufgrund veränderter Aufwachsbedingungen mit der Zunahme erziehungsschwieriger Kinder, fehlender Sozialkontakte durch kleinere Familien, Medienkonsum und Wegfall von Spiel und Bewegungsräumen),

- der Antworten auf die „gewandelten Bildungsanforderungen“ (höhere Abschlüsse, Schlüsselqualifikationen und metakognitive Kompetenzen sowie Orientierungswissen und Medienkompetenz) und

- der „Entwicklung der Lernkultur und der Förderintensität“ (Kompensation von Lerndefiziten durch bessere Diagnostik und Förderkompetenz, Herstellung von Chancengleichheit, Entwicklung einer förderlichen Lern- und Unterrichtskultur für alle erzielen.

Doch der Diskurs endet keineswegs an dieser Stelle. Laut Tillmann sind die zentralen „Reformthemen in der nationalen Reaktion auf PISA [ ] Ganztagsschulen und nationale Bildungsstandards“ (2005, S. 51). Laging fügt hinzu, „dass der Ausbau der Ganztagsschule immer von einer Reform des Unterrichtens unter Einbeziehung von Standards begleitet sein sollte“ (2004, S. 16), um somit eine mögliche Vergleichsebene zu schaffen. Im Folgenden soll erläutert werden, was unter dem Begriff „Bildungsstandard“ verstanden werden kann und wie sich diese explizit zusammensetzen.

\subsection{Bildungsstandards als Instrument der Schulentwicklung}

Die sogenannten Bildungsstandards in den Lehrplänen beziehen sich inzwischen nicht nur auf die „kognitiven“ Fächer wie bspw. Mathematik, Deutsch oder Biologie, sondern seit geraumer Zeit ebenfalls auf die musisch, ästhetischen Fächer wie Kunst, Sport und Musik. Mit den Bildungsstandards und ihren empirischen Überprüfungen soll insbesondere die Schulentwicklung angeregt werden. 
1. Diese Standards fassen die Kerninhalte eines Faches ins Auge und fordern deren intensive Vermittlung. Dies bezieht sich insbesondere auf eine Sicherung von Basiskompetenzen bei schwächeren SuS. Bildungsstandards sollen festlegen, welche (Grund-)Kompetenzen SuS bis zu einer bestimmten Schulstufe erworben haben sollen.

2. Außerdem setzen Standards auf eine empirische Überprüfung des Kompetenzerwerbs, so dass die Frage, was die SuS gelernt haben, nicht länger den Vermutungen verbleibt, sondern eindeutig ermittelt werden kann.

3. Des Weiteren können Schülerleistungen nun miteinander verglichen und bewertet werden. Gründe für Leistungsdifferenzen in Klassen können seitdem mit etwa gleichen Rahmenbedingungen nachgegangen werden. (Tillmann, 2007, S. 36-37)

Eine zielgerichtete Wirkung dieser Standards kann jedoch nur entfaltet werden, wenn die Lehrer/innen zu einer engagierten und motivierten Reformarbeit angeregt werden (Fessler \& Stibbe, 2007). Darüber hinaus sollten die Bildungsstandards als Standards für (sport-) pädagogisches Handeln verstanden werden. Hierbei ist es selbstverständlich wichtig, dass nicht nur die Leistungsergebnisse der SuS berücksichtigt werden. Der Doppelauftrag des Sportunterrichts - die Erziehung und Bildung zum und durch Sport - sollte stets als oberste Maxime von den Lehrenden beachtet werden. Dazu gehört die Fortbildung und das Coaching von Lehrkräften, die Entwicklung individueller Förderpläne sowie ausreichende personale Ressourcen aus quantitativer Sicht, um die gezielte Förderung einzelner SuS gewährleisten zu können.

Zudem sollte die Schule - insbesondere im Ganztagsmodell - einen „Lebens- und Erfahrungsraum“ (Horstkemper, 2005, S. 8) darstellen, in dem die SuS sowohl perfekte Lernbedingungen vorfinden, aber sich ebenso wohl und geborgen fühlen können. Das Gelingen einer solchen Verknüpfung von Standardsetzung, Standardüberprüfung und Qualitätsentwicklung von Schulen und Unterricht würde laut Fessler und Stibbe (2007) auf diese Weise den erhofften positiven Effekt der Standards hervorrufen und zeigen, dass sie sich als sinnvolles Instrument der Schulentwicklung erweisen. 


\subsection{Pädagogische Leistungen von Ganztagsschulen}

Ganztagsschulen haben verschiedene Möglichkeiten, auf pädagogischer Ebene zu fungieren und Leistungen zu erbringen. Sie können laut Holtappels (2009) u.a.:

- die „Lernchancen“ durch individuelle Förderungen verbessern,

- „differenzierte Lehr- Lern- Formen“ durch variable Lernmethoden im Unterricht herstellen,

- „erweiterte Lerngleichheiten und Erfahrungsmöglichkeiten“ durch Lebensweltbezug in fachbezogenen, fächerübergreifenden Formen und durch Schulprojekte anbieten,

- die „Gemeinschaft, soziales und interkulturelles Lernen" durch ein begegnungsförderndes Schulleben mit Festen und Feiern, Theateraufführungen, Tanz-, Musikvorführungen u.v.m. fördern,

- „Partizipation und Demokratielernen" von Schüler(innen) sowie Eltern durch vermehrte Möglichkeiten der Mitbestimmung unterstützen,

- die „gebundene und offene Freizeit im Schulleben“ durch Angebote vor allem im gestalterischen Bereich erweitern und

- "Schulöffnung als differenzierte Arrangements für Lernen und Erfahrung" in inhaltlicher, methodischer und räumlicher (außerschulischer Lernorte) Hinsicht, vor allem auch durch außerschulische Kooperationspartner beschleunigen ( $\mathrm{S}$. $113 \mathrm{ff}$.$) .$

Die Umsetzung der genannten pädagogischen Punkte divergieren allerdings laut Laging, da die Bundesländer teilweise unterschiedliche Konzepte vorweisen und dadurch unterschiedliche Schwerpunkte setzen (Quellenberg, 2007). Es wurde ebenfalls vermerkt, dass anhand der Schülerzahlen an Ganztagesschulen eine erhebliche Divergenz unter den Bundesländern besteht. So weist Thüringen 37,8 \% SuS auf, die eine Ganztagsschule besuchen, während es in Bayern lediglich 3,2 \% sind (Laging, 2014).

Was jedoch positiv nach statistischen Angaben der Kultusministerkonferenz (Züchner, 2010) verzeichnet werden kann, ist eine progressive Entwicklung der Ganztagsschulen. 
So gab es im Schuljahr 2008/2009 mehr als 11.800 schulische Einheiten im Ganztagsbetrieb, was im Vergleich zum Jahr 2002 einen Zuwachs von über $175 \%$ darstellt. Dies bedeutet wiederum, dass innerhalb von sechs Jahren in $42 \%$ aller Schulen Ganztagsangebote vorzufinden sind. Aus statistischer Sicht sind es im Jahr 2009/2010 explizit 13.600 von 34.000 Schulen, die als Ganztagsschulen bezeichnet werden können (Züchner, 2010 S. 5)

Doch welche Voraussetzungen müssen Ganztagsschulen folglich erfüllen? Diesbezüglich sind bislang vergleichsweise wenige Bedingungen verankert worden. Was indes bei der Kultusministerkonferenz 2006 beschlossen wurde, war, dass diese Schulen an mindestens drei Tagen pro Woche geringstenfalls sieben Zeitstunden für ein „Ganztagsangebot“ bereitstellen müssen. An solchen Tagen kommen die Bereitstellung von einem Mittagstisch und ein Nachmittagsangebot hinzu, welches mit dem Vormittagsunterricht ineinandergreift. Die Verantwortung trägt bei allen Angeboten die Schulleitung (KMK, 2006, S. 7).

\subsection{Offene, gebundene und teilgebundene Ganztagsschulen}

Im Folgenden soll erläutert werden, wie sich die drei unterschiedlichen Angebots- bzw. Organisationstypen von Ganztagsschulen unterscheiden.

Die offene Ganztagsschule bietet SuS eine freiwillige Teilnahme an den offerierten Angeboten. Eine Entscheidung, ob an dem halbjährlichen Programm teilgenommen wird oder nicht, findet von den SuS selbst oder deren Erziehungsberechtigten statt. Ob eine tatsächliche Teilnahme an mindestens drei Tagen der Ganztagsangebote seitens der SuS genutzt wird, kann nicht eindeutig gesichert werden. Die Untersuchung „Studien zur Erhebung von Ganztagsschulen“ (2005) ergab, dass an Grundschulen bis zu 80 \% der SuS die Ganztagsangebote wahrnahmen, während an den weiterführenden Schulen nur etwa 30 \% der SuS die Angebote nutzten. Dabei wurden auch nur ausgewählte Angebote an bestimmten Tage besucht (Holtappels 2007, S. 199; Klieme, Holtappels, Rauschenbach \& Stecher, 2007).

Der offenen steht die gebundene Ganztagsschule gegenüber. Hier ist die Teilnahme an mindestens drei Tagen verpflichtend. Dabei wird darauf abgezielt, den SuS eine Struktur hinsichtlich des individuellen Lernbedarfs und der Freizeitbedürfnisse umzusetzen.

Neben diesen beiden erwähnten Formen besteht noch die teilgebundene Form. Bei diesem Konzept nehmen nur bedingt SuS verpflichtend an Angeboten teil. Dies richtet sich häufig an einzelne Klassen oder Klassenstufen. 


\subsection{Begründungen für die Einrichtung von Ganztagsschulen}

Die zentralen Begründungen für die Einrichtung von Ganztagsschulen beruhen größtenteils auf sozial-politische, erzieherische und schulpädagogische Begründungen, welche im Folgenden kurz erläutert werden.

Die sozial-politische Begründung verweist auf das gewandelte Frauenbild und die Veränderung der Institution Familie. Diese Begründung fordert daher eine ganztägige Betreuung der Kinder. Ganztägige Schulen sind laut Höhmann, Holtappels und Schmetzer (2004) als „gesellschaftlicher Beitrag zur Sicherstellung und zur Qualitätssicherung der soziokulturellen Infrastruktur zu verstehen“ (S. 254).

Die erzieherische Begründung zielt auf die Reduzierung der Erziehungsleistung von Familien. Der Grund dafür liegt in der gesellschaftlichen Entwicklung in den letzten Jahren. „Ganztägigen Schulen kommt somit eine kompensatorische Funktion im Bereich des sozialen Lebens und der Sozialerziehung zu“ (Laging \& Hildebrandt-Stramann, 2007, S. 93).

Die schulpädagogische Begründung verweist auf eine Veränderung der Schul-, Unterrichtsund Lernkultur. Der Wandel der Unterrichts- und Lernkultur ist eingebunden in die Frage, wie sich Schule selbst versteht. Daraus entsteht erst die je eigene Schulkultur. Die Schule als Ganztagsschule muss demzufolge ihre eigene Kultur hinsichtlich von Lernen und Leben in der Schule neu finden. Ziel ist es, eine Schulkultur zu entwickeln, die Lernen und Leben umfasst und auf das Besondere der Schule verweist (Laging \& Hildebrandt-Stramann, 2007).

Der Sport kann zudem hervorragende Möglichkeiten der Profilbildung von Ganztagsschulen bieten (ebd.). So können Sport und Bewegung Möglichkeiten der Entspannung und Freizeit im Schulalltag schaffen, was darüber hinaus eine Verbesserung der Konzentrationsfähigkeit und Leistungssteigerung der SuS mit sich bringt (siehe Kapitel 5). Ganztagsschulen müssen jedoch darauf achten, dass sie nicht nur die nach innen ausgerichtete Verbindung von Lernund Lebensraum im Blick haben, sondern auch die Öffnung der Schule nach außen im Sinne einer „Vernetzung inner- und außerschulischer Lebenswelten“ (Fessler \& Stibbe, 2007, S. 9) gewährleisten.

\subsection{Schulkultur und die Bedeutung von außerschulischen Partnern}

Im folgenden Abschnitt soll die Frage erörtert werden, inwieweit Spiel und Sport im Sinne einer fachlichen und überfachlichen Begegnung die Schulkultur mitgestalten können. Laging 
und Hildebrandt-Stramann (2007) glauben, dass die „Bewegung als Weltzugangsweise [ ] hier nicht allein in der fachlichen Konstitution von Unterricht [ ], sondern auch und vor allem als überfachliches Prinzip eines bewegungsbezogenen Umgangs mit dem eigenen Körper, mit der bewegungsherausfordernden Welt und in den Bewegungsbeziehungen mit anderen Menschen“ (S. 101) verstanden werden sollte. Ergo ist Bewegung in einer Ganztagsschule Gegenstand in formellen und nicht-formellen sowie informellen Bewegungsangeboten und anlässen.

Die Gestaltung der unterrichtsfreien Zeiten im Ganztag erfolgt je nach Ganztagsschulkonzeption unterschiedlich. Erstens in Form von freiwilligen Angeboten am Nachmittag in den additiven bzw. offenen Formen der Ganztagsschule. Zweitens in Form verbindlicher Angebote für einen Teil der SuS in teilgebundenen Ganztagschulen. Und drittens in Form eines verbindlichen Angebots für alle SuS in gebundenen Ganztagsschulen (Fessler \& Stibbe, 2007).

An diesen freien Zeiten können externe Einrichtungen oder Experten als Kooperationspartner mitwirken. Im Folgenden werden die bewegungsorientierten Partner vorgestellt, welche aus dem organisierten Sport kommen. Die Thematisierung dieser Kooperationsformen ist aus verschiedenen Gründen von großer Bedeutung:

1. Schulen mit Ganztagsangeboten sind dringend auf Kooperationspartner angewiesen. Der Sport spielt dabei eine wichtige Rolle.

2. Sport und Schule können auf eine lange Tradition durch eine Vielzahl von Kooperationsprojekten zwischen Sportverein und Schule zurückgreifen.

3. Die Sportorganisation, beginnend mit der Spitze des DOSB (Deutscher Olympischer Sportbund) bis hinunter zu den Kreis- und Stadtsportbünden, haben eigene Arbeitsgruppen zum Thema Ganztagsschule eingerichtet. Sie befassen sich u.a. mit den Folgen für die Sportentwicklung und die Vereinsarbeit und möchten die Bewegungssituation von Kindern und Jugendlichen verbessern. (Laging \& Hildebrandt-Stramann, 2007, S. 101-102)

Explizite Kooperationsmodelle zwischen Schule und Sportverein sowie weitere praktische Umsetzungsmöglichkeiten des Fachbereichs Sport in der Ganztagsschule werden in den noch folgenden Kapiteln 8 und 9 aufgezeigt. 


\section{Definitionen grundlegender Begriffe}

Bevor die Wichtigkeit von körperlicher, sportlicher oder allgemeiner Bewegungsaktivität für Kinder und Jugendliche in Deutschland anhand der aktuellen Studienlage aufgezeigt wird, sollten diese Begriffe zunächst definiert werden.

\subsection{Körperliche Aktivität}

Die körperliche Aktivität umfasst „alle körperlichen Bewegungen, die durch den Einsatz größerer Muskelgruppen (Skelettmuskeln) eine substantielle Erhöhung des Energieverbrauchs provozieren“ (USDHHS, S. 16). Dazu zählen u.a. Fußball, Schwimmen, Joggen, Rückengymnastik, Yoga, Tanzen, Radfahren, Treppensteigen, Einkaufengehen, Gartenarbeit und weitere vergleichbare Bewegungsformen.

\subsection{Bewegungsaktivität}

Unter Bewegungsaktivität versteht man alle körperlichen Aktivitäten, die im Alltag ausgeübt werden. Geuter und Hollederer (2012) definieren Bewegungsaktivität als „körperliche Aktivität, die u.a. im Kontext der Erwerbstätigkeit oder der Arbeiten im Haushalt und in der Familie stattfinden sowie Aktivitäten zum Zwecke der Fortbewegung oder des Transports“ (S. 10). Damit ist u.a. Rasenmähen, zu Fuß zur Arbeit gehen, mit dem Rad zur Schule fahren, Putzen oder Treppensteigen gemeint.

\subsection{Sportliche Aktivität}

Woll (1996) definiert sportliche Aktivität bzw. Sportaktivität wie folgt:

„Sportliche Aktivität ist ein aktiver, zielmotivierter, spezifisch organisierter Umgang mit dem Körper innerhalb eines sportlichen Rahmens. Sportliche Aktivität ist immer körperliche Bewegung unter Ausnutzung bestimmter motorischer Fähigkeiten und Fertigkeiten, verbunden mit Befinden und Erleben und eine Form der sozialen Interaktion und Kommunikation." (S. 20)

In dieser Definition mitinbegriffen ist auch der höhere Energieverbrauch, der z.B. bei klassischen Sportarten wie Turnen, Fußball spielen oder Leichtathletik einhergeht. Aber auch al- 
ternativere Sporttätigkeiten wie Tanzen, Nordic Walking oder Yoga können laut dieser Definition im weiteren Sinne als sportliche Aktivitäten verstanden werden.

\section{Sport, Spiel und Bewegung - Ein Grundbedürfnis Kinder und Jugendlicher}

Körperliche Aktivität ist von Kindern und Jugendlichen ein natürliches Grundbedürfnis, welches sich in unterschiedlichster Form repräsentiert. Es moduliert sich vom einfachen „Räuber und Gendarm“ spielen, Klettern \& Inlineskating bis hin zum organisierten „Leistungssport“. Darüber hinaus bietet Bewegungsaktionismus neben der Erkundung der „natürlichen“ Welt einen weiteren Aspekt, nämlich den des Bildens und Entwickelns von kognitiven Fähigkeiten und körperlichen Kompetenzen (Ekeland, Heian \& Hagen, 2005). Das heißt: Kinder, die in frühen Jahren in ihren Bewegungsbedürfnissen eine Förderung erfahren, leiden weniger an Übergewicht, Adipositas, Insulinresistenz, hohem Blutdruck und psychischen Beschwerden gegenüber Kindern, deren Bewegungsausmaß sehr eingeschränkt ist (Freia De Bock, 2012). Diverse Studien belegen, „dass Kinder sich im Vergleich zu vor 20 Jahren objektiv und subjektiv gemessen immer weniger bewegen" (Wilkinson, 2008; Albon, Hamlin \& Ross, 2008; Lampert et al., 2007; McDonald, 2007). Des Weiteren bestätigen Williams et al. (2008) einen Anstieg des Übergewichts im Kindesalter und einen parallelen Rückgang der koordinativen Fähigkeiten. Betroffen sind insbesondere Kinder mit Migrationshintergrund und niedrigem sozioökonomischem Status. Ursachen für diesen Verlauf könnte u.a. die Veränderung der gesellschaftlichen und sozialen Entwicklung sein. De Bock (2012) nennt als zentrale gesellschaftliche und soziale Problemfelder bezüglich des Rückgangs körperlicher Aktivität von Kindern die Motorisierung des Transports, den Medienkonsum und den Rückgang von Bewegungsmöglichkeiten.

\subsection{Bewegungsförderung und Entwicklungsförderung - Zwei untrennbare Komponenten}

In der ersten Lebensdekade bewegen sich Kinder vornehmlich durch spielerische Anregungen. Erhebungen zeigen, dass bei Kindern durch Spielen das eigentliche soziale, kognitive und emotionale Wohlergehen ausgelöst wird (Burdette \& Whitaker, 2005; Ginsburg \& Kenneth, 2007). Pellegrini und Smith (1998) beobachteten, dass durch das Spielen von Kindern positive Auswirkungen sich unmittelbar, aber auch nachhaltig in ihrer Persönlichkeit verankern. Demnach ist Spiel ein unerlässliches Fundament in der Kindes- und Jugendentwicklung. Es muss als wesentlicher Bestandteil betrachtet werden und darf nicht nur als redun- 
dante Präventionsmaßnahme von Übergewicht abgestempelt werden. Bewegungsförderung im Kindesalter bedeutet die Förderung kindlicher Entwicklung (De Bock, 2012).

Doch welche biophysiologischen Prozesse fördern während der Spielbewegung die Entwicklung des jungen Individuums? Durch die Verbindung synaptischer Neuronen im Zentralnervensystem entwickeln Kinder durch Spiel, neben motorischen und koordinativen Fähigkeiten, u.a. Kommunikations- und Sprachkompetenzen. Diese ergeben sich, wenn kooperierende oder Kompromisssituationen im Spiel eintreten. Soziale Dialogfähigkeiten sind weitere Aspekte, wenn es zu Unstimmigkeiten oder Unfairness während des Spielens kommt. In diesem Fall werden Strategien zur Problemlösung eruiert. Selbstbeherrschung, der Ausdruck von Gefühlen und Führungsqualitäten sind jedoch ebenfalls Attribute, die hierbei ausgebildet werden (Eaton, McKeen \& Campell, 2001). Solche Lernsituation treten insbesondere in „bewegten“, also auch sportlichen Interaktionen auf (De Bock, 2012). Sport und Bewegung ist für die Entwicklung von Kindern ergo deutlich vielschichtiger und kann nicht einfach nur auf rein physiologische Präventionsmaßnahmen (Übergewicht bzw. Gesundheit der Kinder und Jugendlichen) reduziert werden.

\subsection{Individuelle Entwicklungsstufen Kinder und Jugendlicher}

Das Vordringen in und Erforschen von Räumen steht häufig in Korrelation mit dem in Kindern und Jugendlichen verankerten intrinsischen Bewegungsbedürfnisses. Allerdings muss bei Schulkindern auch eine hohe Differenzierung des Bewegungsdrangs berücksichtigt werden. Wilkins et al. (2006) haben anhand empirischer Daten herausgefunden, dass es gleichwohl des eingeschränkten Bewegungsbedürfnisses von SuS eine Art individuellen Aktivitätsmodus gibt. Dieser tritt häufig nach Ruhephasen ein, wie bspw. nach der Schule. Zu dieser Erkenntnis kam man, da beobachtet wurde, dass SuS trotz eines zusätzlich implementierten Bewegungsangebots während des Schultags deren Tagesaktivität gleich blieb (Wilkins et. al, 2006). Inwieweit Kinder und Jugendliche sich in Art, Dauer, Intensität und Umfang der körperlichen Aktivität unterscheiden, ist vom jeweiligen Alter abhängig.

Payne und Isaac (2011) konzipierten eine Tabelle, in der veranschaulicht werden soll, welche motorischen Fertigkeiten in welcher Entwicklungs- bzw. Altersstufe eintreten können. Diese variieren jedoch durchaus in der jeweiligen Erscheinungsform interindividuell und sollten deshalb nicht als normativ bewertet werden, sondern lediglich als Orientierungshilfe dienen: 


\begin{tabular}{|c|c|c|}
\hline Entwicklungsstufe & Alter (Jahre) & Fertigkeiten (hohe inter-individuelle Variabilität) \\
\hline Säugling & $0-1$ & $\begin{array}{l}\text { Keine selbstständige Translokation. Rhythmische Stereotypien ohne } \\
\text { erkennbares Ziel, Eltern-Kind-Spiel, Meilensteine }\end{array}$ \\
\hline Kleinkind & $1-4$ & $\begin{array}{l}\text { Spiel mit groben selbstständigen Translokationsbewegungen zur Übung von } \\
\text { Kraft, Ausdauer und Effizienz von Bewegungen }\end{array}$ \\
\hline Frühes Kindesalter & $4-7$ & $\begin{array}{l}\text { Weiter Übungsspiel, Übergang zum Raufen/wilden Spielen bzw. formalen } \\
\text { Spielen in Gruppen ab ca. } 5 \text { Jahren }\end{array}$ \\
\hline Mittleres Kindesalter & $7-9$ & $\begin{array}{l}\text { Raufen/wildes Spielen bzw. formales Spielen in sozialen Gruppen, } \\
\text { kooperatives Spielen: Wichtiger Teil der sozialen Entwicklung der Kinder } \\
\text { (Junge>Mädchen) }\end{array}$ \\
\hline Spätes Kindesalter & $9-11$ & Siehe Mittleres Kindesalter \\
\hline Jugendlichenzeit & $11-21$ & $\begin{array}{l}\text { Ende des Längenwachstums. Bewegung weniger Grundbedürfnis als Teil der } \\
\text { Kultur (z.B. Sport) }\end{array}$ \\
\hline
\end{tabular}

Tab. 1: Lebensspannenkonzept der menschlichen Motorik (Quelle: De Bock, 2012, S. 133)

\section{Zusammenhänge zwischen Bewegung und Gesundheit bei Kindern und Jugendlichen}

\subsection{Psychologische Wirkungen}

Es besteht schon seit längerem insbesondere bei Jugendlichen und jungen Erwachsenen eine nachweisliche Evidenz bezüglich des Zusammenhangs zwischen dem positiven Einfluss von Bewegungsaktivität und psychologischen Wohlbefinden. Beobachtungen zufolge beeinflusst ein sportlich aktives Verhalten Depressionen, Angstzustände und Selbstbewusstseinsprobleme in positiver Hinsicht. Zudem zeigt eine erhöhte Bewegungsaktivität auf soziale Fähigkeiten innerhalb von Gruppen eine stärkende Wirkung (Biddle, Gorely \& Stensel, 2004; Calfas \& Taylor, 1994; Hansen, Larson Dworkin, 2003).

Es gibt allerdings bei Kleinkindern noch nicht genügend hinreichende Studien, die explizit belegen, welche psychologischen Auswirkungen durch Bewegungsaktivitäten hervorgerufen werden. Jedoch haben Ekeland, Heian und Hagen (2005) belegen können, dass Bewegung im Kindesalter positive Effekte auf das Selbstbewusstsein haben. Laut Opper, Worth, Wagner und Bös (2007) sind Kinder, die sich aktiv betätigen und sich in ihrem Bewegungstrieb frei entfalten können, weniger krank und psychisch stabiler. Dementsprechend unterliegen sie deutlich weniger individuell wahrgenommenem Stress im Vergleich zu Gleichaltrigen, die sich wenig bewegen (Hamer, Samatakis \& Mishra, 2009). 


\subsection{Kognitive Fähigkeiten}

Neben einer stärkenden Wirkung auf psychische Faktoren lassen sich durch eine vermehrte Bewegungsaktivität bei Kindern und Jugendlichen ebenfalls mehrere positive Effekte auf deren kognitive Fähigkeiten beweisen. Durch das fortschreitende Bewegungsausmaß im Sinne von Raumerkundung entwickeln Kinder in erste Linie ein besseres Raumverständnis. Dies bedeutet, dass der Sinn für Raumbewusstsein wesentlich geschult wird (Bai und Bertenthal, 1992). Neben dieser Fähigkeit erzielen Kindergartenkinder, die über eine gute Feinmotorik und Hand-Augen-Koordination verfügen, im fortgeschrittenen Alter signifikant bessere Schulergebnisse in Sprachen und Mathematik (Timmons, Naylor und Pfeiffer, 2007).

Auch können bei Kindern und Jugendlichen, die sich verstärkt bewegen, sowohl kurzzeitige Verbesserungen der Konzentrationsfähigkeit, als auch langfristig verbesserte Schulergebnisse ermittelt werden (Reed et al., 2010). Diese Korrelation ist häufig ebenfalls mit der Ausdauerleistungsfähigkeit, welche durch regelmäßige körperliche Aktivität einhergeht, verbunden. Diese schafft wiederum eine Verbesserung der Konzentrationsfähigkeit bei Kindern und Jugendlichen. Bei konzentrationsintensiven Phasen wie bspw. analytischem Lesen von Texten, der Aneignung von unbekannten Sachverhalten, Präsentationen und Klassenarbeiten stellen eine ausgeprägte Ausdauerleistungs- und die damit einhergehende Konzentrationsfähigkeit für SuS einen unerlässlichen Vorteil dar. Hillman, Erickson und Kramer (2008) sowie Hillman und Schott (2013) bestätigen, dass zwischen körperlicher Aktivität bzw. einer besseren Fitness ein „positive[r] Zusammenhang [...] mit der schulischen Leistungsfähigkeit“ (S. 34) besteht. Dergleichen konstatieren Sibley und Etnier (2003) in ihrer Meta-Analyse. Darin bewerten sie ebenfalls positive Korrelationen von körperlich-sportlicher Aktivität und kognitiver Leistungsfähigkeit bei vier bis 18-jährigen Schulkindern.

Zusätzlich wurde erkannt, dass hohe Fitness wiederum die neurologisch zerebrale Entwicklung bei Kindern und Jugendlichen fördert. So haben Chaddock, Erickson, Prakash, Kim, Voss, Van Patter und Kramer (2010) anhand bildgebender Magnetresonanztomographien (MRT) festgestellt, dass bei Kindern zwischen neun und zehn Jahren mit hohem Fitnessgrad gegenüber Kindern mit geringer Fitness ein physiologischer Unterschied in der Gehirnstruktur ersichtlich ist. Kinder mit hoher Fitness wiesen ein größeres Hippocampusvolumen auf als Kinder mit verminderter Ausdauerleistungsfähigkeit. Der Hippocampus ist u.a. für die Einspeicherung neuer Gedächtnisinhalte und das Überführen von Informationen vom Kurz- ins Langzeitgedächtnis zuständig. Infolgedessen ist er maßgeblich an Lernprozessen beteiligt. Zudem ermittelten Chaddock et al. (2010) bei Kindern mit hoher Ausdauerleistungsfähigkeit 
beeindruckende Ergebnisse in ihren Untersuchungen bzgl. relationaler Gedächtnistests. Zu vergleichbaren Ergebnissen im Zusammenhang mit der Ausdauerleistungsfähigkeit und dem hippocampalen Volumen kamen ebenfalls Chaddock, Hillmann, Buck und Cohen (2011) wenngleich sich ihre Forschungsarbeit auf Jugendliche bezog.

Neben der rein biophysiologischen Strukturentwicklung wird anhand weiterer Studien vermutet, dass ein positiver Effekt von körperlich sehr fitten Kindern auf größere Aufmerksamkeitskapazitäten zur Reizverarbeitung besteht. Das kann sich zum einen durch verbesserte motorische Willkürbewegungen (wichtig bei Sportspielen) und bei kognitiven Prozessen, zum anderen durch reine Aufmerksamkeitskontrolle ausdrücken (Colcombe \& Kramer, 2003; Etnier \& Chang, 2009; Hilmann et al., 2008). Hillman und Schott (2013) bewerten dies folgendermaßen:

„Es lässt sich zusammenfassend festhalten, dass positive Effekte einer einzelnen Ausdauertrainingseinheit auf Aufmerksamkeitsaspekte der kognitiven Kontrolle bestehen, die die Nutzung von Aufmerksamkeitsressourcen in alters- und übungsabhängiger Weise zu erleichtern scheinen. Obwohl die Nachweise nur spärlich sind, zeigen vorläufige Ergebnisse, dass intensive Übungseinheiten die schulische Leistungsfähigkeit verbessern können und daher einen wichtigen Gesichtspunkt für die schulische Praxis sowie das Klassenklima darstellen. Erst kürzlich wurden in unserem Labor die Effekte einzelner Trainingseinheiten auf neurokognitive Tests, Verhaltenstests und schulische Leistungstests repliziert und diese Befunde auf Kinder mit Aufmerksamkeitsdefizit-/ Hyperaktivitätsstörung (ADHD) ausgeweitet." (S. 38-39)

Diese Studienergebnisse legitimieren den Sport als Schulfach nicht einfach nur - sie zeigen darüber hinaus auf, dass durch Sport, Spiel und Bewegung für Kinder und Jugendliche in mehrerlei Hinsicht unglaubliche Chancen und Möglichkeiten geschaffen werden können.

Diese würden im Umkehrschluss ebenfalls eine enorme Hilfe und Entlastung für die Lehrkräfte und Eltern darstellen. Nichtsdestotrotz müssen weitere Studien folgen, damit die laut Hillman und Schott (2013) spärlichen Nachweise handfester werden und konkretere Empfehlungen ausgesprochen werden können. 


\subsection{Physiologischer Einfluss}

Dass Bewegungsmangel negative Auswirkungen auf die Gesundheit von Erwachsenen hat, ist absolut kein Novum. Mit dem Altern treten simultan erste degenerative Prozesse ein. Diese werden durch körperliche Inaktivität nicht selten beschleunigt. Neben auftretenden HerzKreislauf-Erkrankungen, sind Diabetes-Mellitus II, Osteoporose und Rückenschmerzen weitere medizinische Krankheitsphänomene (Sallis \& Owen, 1998). Doch diese medizinischen Auswirkungen beschränken sich nicht nur auf Erwachsene, denn auch bei Kindern mit Bewegungsmangel konnten neben erhöhten Blutfettwerten ebenso erhöhte Blutdruckwerte belegt werden (Hernelathi, Kujala \& Kaprio, 2004; Hunt et al., 2011).

Neben den organischen Verschlechterungen können auch Defizite in der knöchernen Struktur bei Heranwachsenden eintreten. Im Verlauf des Kindesalters bildet sich die Knochenstruktur. Eine erhebliche Beeinflussung der Knochendichte wird durch Scher- und Druckkräfte verursacht. Ausgelöst werden diese durch körperliche Aktivitäten wie bspw. springen, rennen oder hüpfen. Daher sind auch Übungsleiter/innen, Sporttrainer/innen und Sportlehrer/innen dazu sensibilisiert, im Kindesalter dementsprechend altersgerechte Übungen zu implementieren, da die Knochen noch sehr flexibel sind. Um andererseits einen Verdichtungsreiz des Knochens hervorzurufen ist es nötig, dass er durch Spiel und Bewegung trainiert und gefördert wird. Allein eine täglich zehnminütige, körperlich anspruchsvolle Aktivität, kann die Mineralisierung des Knochens um drei Prozent verbessern und somit Osteoporose entgegenwirken (De Bock, 2012).

Als einen weiteren zu erwähnenden Aspekt ist die muskuläre Struktur zu nennen. Die Skelettmuskulatur befähigt den Menschen überhaupt erst dazu, biomechanische Bewegungsabläufe zu generieren. Nur so kann sich ein Mensch fortbewegen. Durch Sport und Bewegung werden unterschiedliche Bereiche der Muskulatur beansprucht. Erst anhand der biomechanischen Reize entsteht eine Kräftigung der Muskulatur. Die Muskulatur, welche eine der wichtigsten Organe des Menschen darstellt, kann z.B. unseren Körper bei Sprüngen und Landungen auf Unebenheiten stabilisieren, Richtungsänderungen bei Spielsportarten einleiten oder aber Gelenke bzw. innere Organe vor äußeren mechanischen Einwirkungen schützen wie bspw. bei einem Sturz von einem Skateboard oder bei körperbetonten Kampfsportarten (Dickhut, 2000).

Ergo kann durch körperliche Aktivität eine Vielzahl positiver Effekte erreicht werden, welche den Menschen vor Krankheiten bewahrt oder im Alltag ungemein unterstützt. Auf der ande- 
ren Seite besteht bei jeglicher Ausführung sportlicher Aktivitäten immer die Gefahr, sich selbst Verletzungen zuzufügen. Dickhuth (2000) ist jedoch der Meinung, dass die unzähligen Chancen auf bspw. die Verbesserung der Gesundheit, des Lebensstils, der kognitiven Leistungsfähigkeit oder des psychischen Wohlbefindens die Gefahren und negativen Effekte immer bei weitem übertreffen.

\section{Kinder und Jugendgesundheitssurvey (KiGGS)}

Im Folgenden wird anhand des Kinder und Jugendgesundheitssurveys („KiGGS“), welcher im Auftrag des Robert Koch-Instituts ausgeführt wurde, erläutert, wie im Zusammenhang mit dem umgreifenden Rückgang von körperlich-sportlicher Aktivität die konkrete Sachlage bei deutschen SuS aussieht. Nach Lampert et al. (2007) „stellt nun (KiGGS) erstmalig umfassend belastbare Daten zur körperlich-sportlichen Aktivität der heranwachsenden Generation in Deutschland bereit und schließt damit die vorhandene Datenlücke“ (S. 635).

Die Erhebung fand von Mai 2003 bis Mai 2006, ergo über einen Zeitraum von drei Jahren statt. Das Ziel war anhand einer bundesweit umfassenden Datenlage den Gesundheitszustand der sich im Alter von 0 bis 17 Jahren befindlichen Kinder- und Jugendlichen zu ermitteln. Bei dieser Untersuchung haben insgesamt 17.641 Kinder und Jugendliche teilgenommen - davon 8.656 Mädchen und 8.985 Jungen, die wiederrum aus 167 Städten und Ortsgemeinden stammten.

Die Daten zur körperlich-sportlichen Aktivität wurden anhand von Fragebögen gesammelt. Dabei lautete die Fragestellung bei den 11- bis 17-Jährigen, wie häufig sie in ihrer Freizeit aktiv sind (z.B. Fahrrad fahren oder Sport treiben). Als Zusatz wurde die Begrifflichkeit „aktiv“ genauer definiert: Aktiv bedeutet, dass sie außer Atem oder richtig ins Schwitzen kämen. Zu dieser Frage gab es fünf Antwortmöglichkeiten: „nie“, „etwa ein bis zwei Mal im Monat“, "etwa ein bis zwei Mal in der Woche“, „etwa drei bis fünf Mal in der Woche“ und „etwa jeden Tag". Zudem sollten die befragten Kinder und Jugendlichen eine grobe Einschätzung geben, wie viele Stunden sie pro Woche bewegungsaktiv sind. Bei den 3- bis 10-jährigen Kindern wurde die Beurteilung der körperlichen Aktivität anhand der Auskünfte der Erziehungsberechtigten erfragt. So wurden insgesamt 7.698 Daten ermittelt. Der Hinweis, dass zwischen Vereins- und Freizeitsport differenziert werden sollte, wurde ebenfalls gegeben.

Bei den Kindern und Jugendlichen im Alter von 11 bis 17 Jahren kam es zu einem Gesamtbestand von 6.667 Daten und somit zu einer Teilnehmerquote von 66,6 \%. Um eine strukturierte und explizierte Auswertung vornehmen zu können, wurden verschiedene Unterteilungen 
vorgenommen. So wurden die Ergebnisse nach Alter, Geschlecht, Migrationshintergrund, sozialem Status und Wohnregion analysiert und unterteilt. Die Eltern wurden nach schulischem und beruflichem Werdegang befragt. Hinzu kamen Angaben zu ihrem Einkommen und ihrer beruflichen Position. Anhand dieser Angaben konnten die befragten Familien in untere, mittlere und hohe "Statusgruppen“ kategorisiert werden (Lange, Kamtsiuris, Lange et al., 2007).

Innerhalb dieser KiGGS-Studie wurde außerdem durch die verpflichtende Angabe der Eltern über das Geburtsland und der Staatszugehörigkeit der Kinder sowie der Eltern selbst, der exakte Migrationshintergrund der Probanden ermittelt. Nach Lampert et al. (2007) werden als Migranten „Kinder und Jugendliche bezeichnet, die selbst aus einem anderen Land zugewandert sind und von denen mindestens ein Elternteil nicht in Deutschland geboren ist, oder von denen beide Eltern zugewandert bzw. nicht deutscher Staatsangehörigkeit sind" (S. 635). Der Wohnort zum Zeitpunkt der Erhebung wurde darüber hinaus zwischen alten und neuen Bundesländern unterschieden. Denn relevant ist immer nur der gegenwärtige Wohnort der Kinder und Jugendlichen zum Zeitpunkt der Studie (Lange et al., 2007).

\subsection{Ergebnisse der sportlichen Aktivität von Kindern zwischen 3 und 10 Jahren}

Laut der ermittelten Ergebnisse treiben 75 \% der 3- bis 10-jährigen Mädchen und 76,6 \% der Jungen mindestens einmal pro Woche Sport. Dies schließt den Vereins- und Freizeitsport mit ein. Mindestens drei Mal oder mehr pro Woche treiben 43,1 \% der Jungen und 36,2 \% der Mädchen Sport. Mit Zunahme des Alters steigt die sportliche Aktivität. Bei 10-jährigen Jungen und Mädchen wurde eine Teilnahme am Sport von 84,4 \% bzw. 76,7 \% ermittelt. Diejenigen Kinder, welche beständig sportlich aktiv sind, weisen zudem einen Anstieg ihrer Aktivitäten von mehr als drei Mal oder häufiger pro Woche auf. Diesbezüglich sind es insbesondere bei den 10-Jährigen zwei Drittel des männlichen Geschlechts und ca. die Hälfte des weiblichen Geschlechts, welche sich vermehrt sportlich aktivieren (Lampert et al., 2007).

Nahezu 75 \% der Kinder von 3 bis 10 Jahren sind im Vereinssport eingebunden. Die Werte aller Jungen und Mädchen, die mindestens einmal in der Woche vereinsaktiv sind, liegen bei 57,0 \% bzw. 55,0 \%. Dem gegenüber stehen nur 9,1 \% der Jungen und 5,0 \% der Mädchen, die drei Mal oder häufiger pro Woche im Verein aktiv sind. Jedoch ist nicht zu verkennen, dass Kinder auch außerhalb des Vereins sportlich aktiv sind. So betätigen sich $52,4 \%$ der Jungen und 48,0 \% der Mädchen mindestens einmal pro Woche, ohne vereinsaktiv zu sein. Bezüglich der körperlichen Aktivität von mindestens drei Mal und häufiger pro Woche au- 
ßerhalb des Vereins beziffert sich die Datenlage auf $24,2 \%$ bei Jungen und 16,6 \% bei Mädchen.

Die Ergebnisse lassen eindeutig eine Verdichtung der Sportaktivität mit dem Altersanstieg erkennen - ob innerhalb oder außerhalb eines Vereins, die Studienergebnisse sind hierauf Bezug nehmend kongruent. Durch den Vereinssport wird die sportliche Aktivität jedoch besser ersichtlich. Die Mehrzahl der Kinder, die Vereinssport betreiben, ist ohnehin bereits im Alltag sportlich aktiv.

Nun sollte jedoch geklärt werden, wie es sich mit denjenigen Kindern verhält, die sich nicht körperlich betätigen? Die KiGGS-Studie offenbart anhand der erhobenen Datenlage, dass in den Altersgruppen der 3- bis 10-Jährigen etwa jedes vierte Kind unregelmäßig Sport treibt und jedes achte Kind sich gar nicht sportlich betätigt.

Um in diesem Bereich Gegenmaßnahmen in Form von Sportprogrammen und Sportförderungen konzipieren zu können, erwägt „Public Health“, das anwendungsorientierte Fachgebiet, welches sich mit der öffentlichen Gesundheit befasst, eine möglichst präzise Beschreibung der Betroffenen. Anhand der ersten Daten wird ersichtlich, welche Gruppen davon betroffen sind und welche Differenzierung nach Wohnregion, Migrationshintergrund und Sozialstatus ersichtlich ist. Ersichtlich wird, dass die Altersklasse der Jungen von 3 bis 10 Jahren, welche sich weniger als einmal pro Woche (im Verein oder außerhalb) sportlich aktivieren, 36,2 \% der niedrigsten Statusgruppe zuzuordnen sind. 21,0 \% bzw. 14,9 \% der Jungen dieser Altersklasse gehören hingegen der mittleren bzw. höheren Statusgruppe an.

Bei den Mädchen verhält es sich hinsichtlich der sportlichen Aktivität nicht anders. Hier wird ersichtlich, dass 40,4 \% der Mädchen der niedrigsten bzw. 24,1 \% und 12,1\% der mittleren und höheren Statusgruppe angehörig sind. Ferner wird belegt, dass Bewegungsinaktivität häufiger bei Kindern mit Migrationshintergrund bzw. bei Kindern aus den neuen Bundesländern auftritt.

Anhand der folgenden Tabelle kann aufgezeigt werden, wie sich der bereits erläuterte Anteil der 3- bis 10-jährigen Jungen und Mädchen, die weniger als einmal pro Woche Sport machen, nach Sozialstatus, Migrationshintergrund und Wohnregion im Expliziten zusammensetzt: 


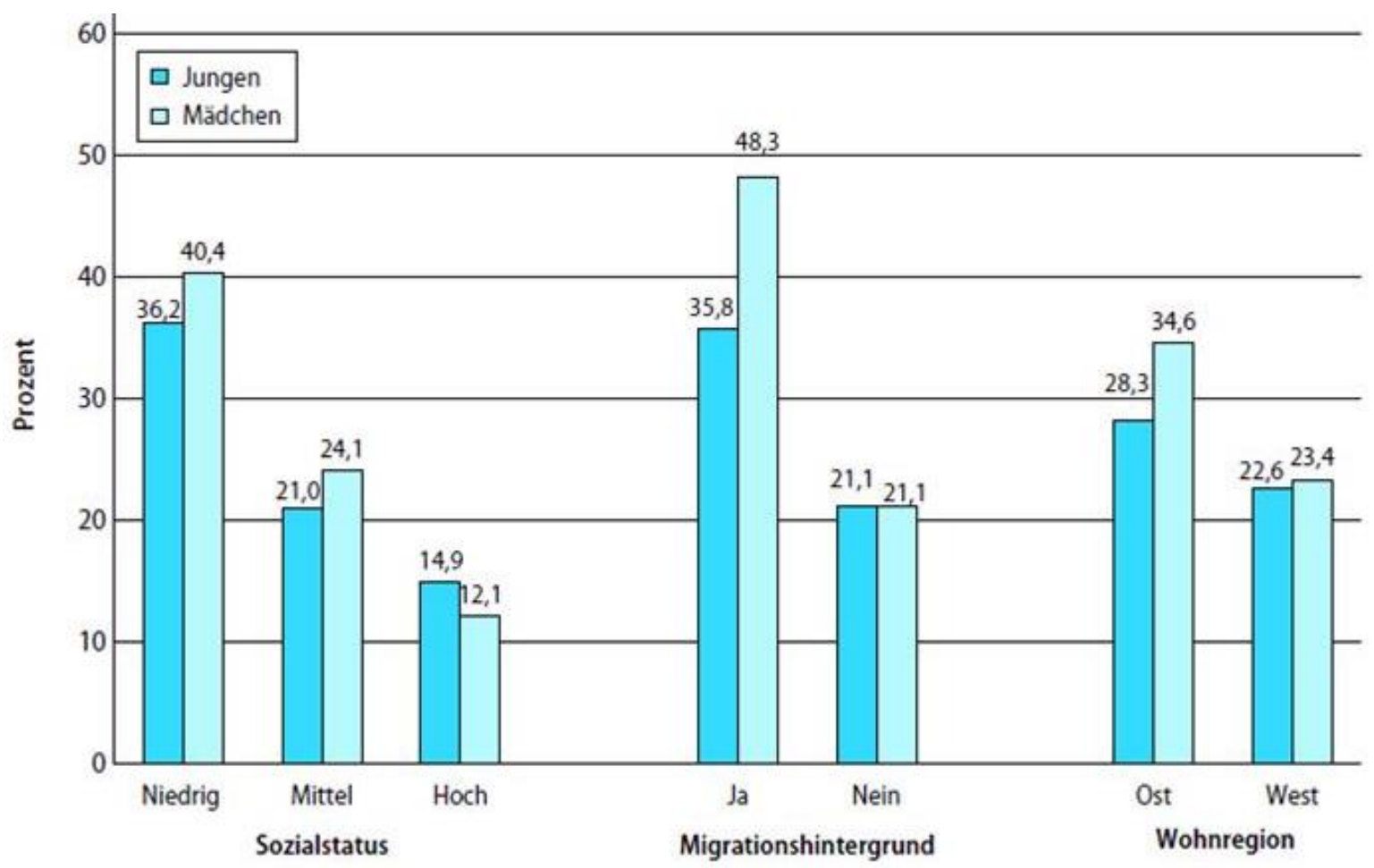

Abb. 1: Anteil der 3- bis 10-jährigen Jungen und Mädchen, die weniger als einmal pro Woche Sport machen, nach Sozialstatus, Migrationshintergrund und Wohnregion (Quelle: Lampert et al., 2007, S. 638)

\subsection{Ergebnisse der sportlichen Aktivität von Jugendlichen zwischen 11 und 17 Jahren}

Auf die Frage „Wie häufig bist Du in deiner Freizeit körperlich so aktiv (z.B. beim Sport oder Fahrradfahren), dass Du dabei richtig ins Schwitzen kommst?" beantworteten 89,9 \% der 11 bis 17-jährigen Jungen, dass sie mindestens einmal in der Woche für derartige Aktivitäten Zeit investieren. Bei den Mädchen liegt der Aktivitätsbereich bei 78,5 \%. Die formulierte Mindestempfehlung von drei Mal oder häufiger pro Woche sportlicher Aktivität gehen 64,7 \% der Jungen und lediglich 43,3 \% der Mädchen nach. Täglich aktiv sind durchschnittlich nur 28,2 \% der Jungen und 17,3 \% der jugendlichen Mädchen von 11 bis 17 Jahren. Während bei Kindern noch keine signifikanten geschlechtsspezifischen Unterschiede auftreten, was die sportliche Aktivität betrifft, so wird dies bei den Jugendlichen evidenter.

Mit Zunahme der Adoleszenz nimmt die körperliche Aktivität der Mädchen deutlicher ab, als es bei den Jungen der Fall ist. Alarmierend ist auch, dass mit zunehmenden Alter beider Geschlechter die tägliche sportliche Aktivität rapide sinkt. Bei den 17-Jährigen sind es nur noch 18,4 \% der Jungen und 11,2 \% der Mädchen, die sich fast täglich sportlich betätigen. 


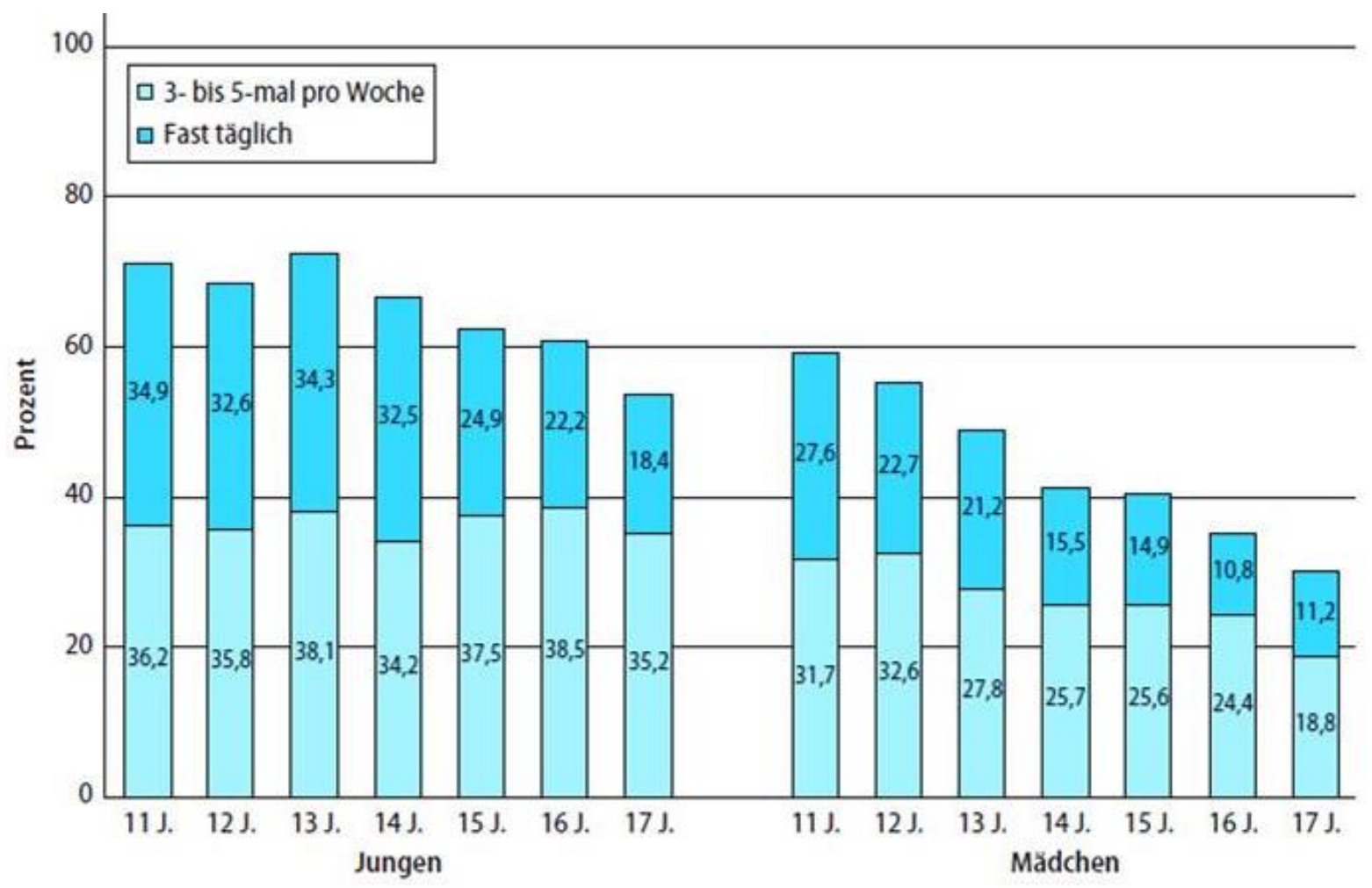

Abb. 2: Abnahme der körperlich-sportlichen Aktivität bei 11- bis 17-jährigen Jungen und Mädchen (Quelle: Lampert et al., 2007, S. 639)

Lampert et al. (2007) formulieren diesen drastischen Rückgang folgendermaßen:

„Die alters- und geschlechtsspezifischen Unterschiede in der körperlichsportlichen Aktivität von Jugendlichen lassen sich auch mit dem wöchentlichen Stundenumfang belegen. Im Durchschnitt verwenden die 11- bis 17-jährigen Jungen 7,8 Stunden pro Woche auf körperlich-sportliche Aktivitäten, die gleichaltrigen Mädchen hingegen nur 4,5 Stunden. Bei Jungen nimmt der wöchentliche Zeitaufwand im Altersgang von 8,0 bei den 11- bis 17-Jährigen nur leicht ab, bei Mädchen geht er deutlich zurück, und zwar von 5,1 auf 4,2 Stunden pro Woche“ (S. 639).

Jugendliche, die sich weniger als einmal pro Woche körperlich-sportlich aktivieren, werden von Lampert et al. (2007) als „inaktiv“ bezeichnet. Im Altersbereich der 11- bis 17-Jährigen bestätigt sich dies aufgrund der durchschnittlichen Werte von 10,1 \% (Jungen) und 21,5 \% (Mädchen). In der Altersgruppe der 17-Jährigen ist sogar jeder sechste Junge und bereits jedes dritte Mädchen dieser Gruppe zuzuordnen. Gegenüber der 3- bis 10-Jährigen lassen sich in der Altersklasse der 11- bis 17-jährigen Jugendlichen weniger Zusammenhänge erkennen, welche die sportliche Inaktivität mit dem Migrationshintergrund, Sozialstatus und 
der Wohnregion begründet werden könnte. Ergo kann dokumentiert werden, dass die Inaktivität bei jugendlichen Jungen in der niedrigsten und mittleren Statusgruppe bei $10,3 \%$ und 10,2 \% liegt - entgegen dem höchsten Status, dessen Wert sich auf etwas niedrigere 8,9 $\%$ beziffert. Marginal sind ebenfalls die Korrelationen von Migrationshintergrund und Wohnregion (alte und neue Bundesländer).

Konträr verhält es sich indessen bei den weiblichen Jugendlichen. Dort kann laut der KiGGSStudienergebnisse (Lampert et al, 2007) beobachtet werden, dass ein niedriger Sozialstatus durchaus zu einer erhöhten sportlichen Inaktivität verstärkend beiträgt. Folglich beziffert sich der Wert sportlich inaktiver Mädchen dieser Altersklasse bei der niedrigsten Gruppe auf $28,1 \%$ - in Relation dazu taxieren sich die Werte der mittleren und höchsten Statusgruppen nur auf 20,2 \% und 15,8 \%. Zu vermerken ist zusätzlich, dass zum Zeitpunkt der Untersuchung im Jahr 2007, Mädchen mit Migrationshintergrund aus den neuen Bundesländern häufiger inaktiv sind, als Mädchen ohne Migrationshintergrund, wenn diese in den alten Bundesländern ihren Wohnsitz haben. Allerdings lässt sich die Wirkung der Faktoren „Migrationshintergrund", "Sozialstatus" und „Wohnregion" auf eine sportliche Inaktivität nur aussagekräftig bei Mädchen verzeichnen. Was jedoch bei beiden Geschlechtern attestiert werden kann, ist der wesentliche Rückgang der körperlich-sportlichen Aktivität mit zunehmendem Alter.

Daraus kann folgende Schlussfolgerung gezogen werden: Mit der Zunahme des Alters besteht schon bei den 11 bis 17-jährigen Jugendlichen mit jedem Lebensjahr eine 30 \% höhere Wahrscheinlichkeit, in die sportliche Inaktivität zu geraten (Lampert et al., 2007).

\subsection{Fazit und Diskussion}

Die ersten Daten der KiGGS-Studie aus dem Jahr 2007 lieferten folgende Zahlen: 75 \% der 3bis 10-jährigen Kinder sind mindestens einmal pro Woche aktiv. Ein Drittel dieser Altersgruppe ist bis zu drei Mal oder häufiger pro Woche sportlich aktiv. Dem gegenüber stehen allerdings $25 \%$ der Kinder, die ernstzunehmende sportliche Aktivitätsdefizite vorweisen. Die gesammelten Daten konnten allerdings zum gegenwärtigen Zeitpunkt noch keine explizite Beurteilung zulassen, inwiefern sich Kinder tatsächlich zu wenig bewegen. Hierzu waren laut Kurth (2006) die gesammelten Daten dieser Altersgruppe nicht umfassend und detailliert genug. Dies lag bestimmt auch an der Vorgehensweise, dass die Erziehungsberechtigten die Beurteilung der körperlichen Aktivität ihrer Kinder übernahmen. 
Um objektivere Aussagen treffen zu können, müsste darüber hinaus neben dem Parameter der sportlichen Aktivität auch der Parameter der täglichen körperlichen Aktivität berücksichtigt werden. Diese Informationen sollte eine weitere Studie innerhalb der KiGGS- Studie liefern: Das Motorik-Modul („MoMo“). Die Ergebnisse dieser Untersuchung werden im folgenden Kapitel vorgestellt. So viel soll jedoch vorab erwähnt werden: Das Motorik-Modul betrachtet die Organisationsform, Häufigkeit, Dauer und Intensität der sportlichen Aktivität. Der Schulsport ist hierbei mitinbegriffen. Ferner sollen diese Resultate explizitere Informationen des körperlichen Bewegungsverhaltens zur Verfügung stellen (Opper et al., 2006). Im Gegensatz zur Datenlage der Altersgruppe der Kinder ist die Beurteilung der körperlichen Aktivität bei den Jugendlichen von 11 bis 17 Jahren deutlich besser gegeben. Zum einen wurde die Fragestellung hier nicht nur auf die sportliche, sondern eben auch auf die körperliche Aktivität ausgerichtet und zum anderen orientierte man sich eng an der zu diesem Zeitpunkt schon bestehenden Bewegungsempfehlung. Die Resultate veranschaulichen, dass sich der Großteil der Jugendlichen zumindest einmal pro Woche sportlich aktiviert. Betrachtet man indes die Bewegungsaktivität "drei Mal pro Woche und mehr" so wird von einem Drittel der Jungen und gar von der Hälfte der Mädchen diese medizinische Gesundheitsempfehlung nicht erreicht (Pate, Pratt \& Blair, 1995).

Dieses bereits erwähnte Mindestmaß an physischer Betätigung wurde als Empfehlung für erwachsene Personen formuliert. Für Kinder und Jugendliche hingegen wird innerhalb der Forschung eine Empfehlung von mindestens einer Stunde am Tag ausgesprochen (Cavill, Biddle \& Sallis, 2001; Prochaska, Sallis \& Long, 2001). So erfüllen laut der KiGGS-Ergebnisse der 11- bis 17-jährigen Jugendlichen ausschließlich jeder vierte Junge bzw. jedes sechste Mädchen diesen Mindeststandard. Geben die ersten Erhebungen noch keine vollständige Auskunft der zu untersuchenden Parameter, so liefern sie dennoch hinreichende Indizien, um konzeptionelle Überlegungen für den körperlich-sportlichen Aktivitätsbereich insbesondere von Jugendlichen zu generieren. Auffallend ist der signifikante Aktivitätsunterschied bei den 14- bis 17-jährigen Mädchen gegenüber den Jungen dieser Altersspanne. Bei exakt diesen Altersgruppen klaffen die Anforderungen und Empfehlungen von der Realität am weitesten voneinander ab und das sowohl bei den Mädchen als auch bei den Jungen. Folglich sind Alter und Geschlecht wichtige Parameter, die bei zukünftigen Untersuchungen noch expliziter berücksichtigt werden können. Eine sogenannte gruppenorientierte Vorgehensweise ist also ratsam. 
Gleichermaßen sollte bei den 3- bis 10-jährigen Kindern vorgegangen werden. Diese Altersgruppe wird im Gegensatz zu den Jugendlichen doch eher durch die Parameter „Migrationshintergrund", "Sozialstatus" und "Wohnregion" beeinflusst. Auffallend ist außerdem, dass Kinder, die in Sportvereinen aktiv sind, sich deutlich häufiger körperlich betätigen als Kinder, die in keinen Vereinen angemeldet sind. Die Begründungsversuche könnten dahingehend so gedeutet werden, als dass Kinder aus sozialökonomisch benachteiligten Verhältnissen seltener die teilweise erheblichen Mitgliedsbeiträge von Sportvereinen aufbringen können. Hinzu kommen häufig noch Kosten für Sportutensilien und Vereinsausflüge.

Gleichermaßen ist es bei Familien mit Migrationshintergrund zu sehen, deren finanzielle Situation sich überwiegend ebenfalls an der Armutsgrenze ansiedelt (Bundesministerium für Gesundheit und soziale Sicherung, 2005). Je nach ethnischer Herkunft muss berücksichtigt werden, dass neben dem finanziellen Aspekt auch noch die religiöse und kulturelle Herkunft besonders bei Mädchen eine Rolle spielt. Bedenken der Eltern von bspw. muslimischen Kindern bzgl. gemeinschaftlicher sportlicher Aktivitäten in Vereinen - den Schulsport mitinbegriffen - sind durchaus vorhanden.

Bei der Betrachtung der Vereinsaktivität im Zusammenhang mit der Ost-WestWohnsituation ist zu vermerken, dass nach dem Fall der Mauer eine beträchtliche Anzahl an Sportinstitutionen in der ehemaligen DDR wegfielen (Pfeifer, 2003). Überdies könnten die Vorstellungen des damaligen äußerst leistungsfokussierten Sportsystems in Ostdeutschland eine Ursache dafür sein, dass Eltern in der Folgezeit ihre Bedenken hinsichtlich des institutionalisierten Vereinssports nach 1990 zum Ausdruck brachten, indem sie ihre Kinder nicht dort anmeldeten.

Wie bereits erwähnt wäre es für jeglichen Bereich des Sports sehr empfehlenswert, eine gruppendifferenzierte Berücksichtigung umzusetzen, damit Verbesserungen der körperlichsportlichen Aktivität von Kindern und Jugendlichen eintreten können. Diese Maßnahmen lassen sich möglicherweise nicht durch gesonderte, sondern nur durch diverse aufeinander bezogene und ausgearbeitete Programme erreichen. Insbesondere die Schulen und Vereine in Deutschland können hierzu einen bedeutsamen Beitrag leisten. Das Hauptziel sollte sein, dass insbesondere Kinder mit Migrationshintergrund oder aus Familien mit niedrigem Sozialstatus sowie Jugendliche zwischen 14 und 17 Jahren eine größere Bewegungsfreude vermittelt bekommen. Dies impliziert wiederum ein gut geschultes und qualifiziertes Personal an Übungsleitern und Sportlehrern (Leppin, Kollip \& Hurlemann, 1996; Hoffmann, Brandt \& Schlicht, 2006). 
Weiterhin wären mediale Verlautbarungen und Aufrufe hilfreich, die neben den elementaren gesundheitlichen Perspektiven auch weitere Nutzen, wie Ausgeglichenheit, Freundschaften und Spaß am Sport treiben, verdeutlichen (Bundeszentrale für gesundheitliche Aufklärung 2003). Bzgl. dieser vorgeschlagenen Maßnahmen darf keineswegs in Vergessenheit geraten, welche Relevanz dem Bestand bzw. dem Ausbau von weiteren Spiel- und Bewegungsräumen zugeschrieben wird, welche vorrangig in den Großstädten zurückgehen (Fuchs, 2003).

Innerhalb dieser Arbeit wird im Kapitel 10 auf diese Problematik konkreter eingegangen. Auch die Möglichkeiten einer Ganztagsschule auf die Felder „Bewegung, Spiel und Sport“ sowie damit einhergehende, neugeschaffene Bewegungsräume innerhalb der Institution Schule bergen vielversprechende positive Effekte auf die körperlich-sportliche Aktivität von Kindern und Jugendlichen. Hierauf wird im weiteren Verlauf dieser Abschlussarbeit ebenfalls noch expliziter eingegangen.

\section{Motorik-Modul-Studie (MoMo)}

Im Kindes- und Jugendalter tragen körperlich-sportliche Aktivitäten wesentlich dazu bei, dass Heranwachsende sich aus medizinischen und sozialen Gesichtspunkten gesund entwickeln können. Motorische Bewegungen sind hierfür grundlegende Voraussetzungen, um diese und weitere Prozesse positiv zu unterstützen und bestmöglich zu fördern. Die Motorik-Forschung macht es sich u.a. zur Aufgabe, der Frage nach dem tatsächlichen motorischen Leistungszustand von Kindern und Jugendlichen nachzugehen, diesen zu erfassen und wenn möglich zu bewerten. Des Weiteren wird neben diesem Untersuchungsgegenstand innerhalb dieser Abschlussarbeit die aktuelle Datenlage mit der von früheren Generationen verglichen und evaluiert. Zudem werden zukünftige Tendenzen kritisch reflektiert und diskutiert.

Hinsichtlich der Leistungsfähigkeit prallen teilweise kontrovers diskutierte Ansichten aufeinander, inwieweit sich die „Jugend von heute“ noch genügend bewegt. Im Vergleich zu früheren Generationen bestehen unterschiedliche Ansichten über deren motorische Leistungsfähigkeit. Einerseits wird bei Kindern und Jugendlichen zwischen 6 und 10 Jahren ein Rückgang sportlicher Leistungsfähigkeit konstituiert. Andererseits ist bei Kinder und Jugendlichen im Alter von 11 und 12 Jahren von unveränderten Werten die Rede. Die Datenlage ist demnach diffus und erlaubt große Interpretationsspielräume.

Im Zuge der KiGGS-Studie wurde die Motorik-Modul-Studie („MoMo“) implementiert. Sie liefert neben den rein durch Fragebögen erhobenen Messdaten der KiGGS-Untersuchungen 
eine zusätzlich motorisch-praktisch fundierte Datenmenge. Dadurch wird die KiGGSErhebung durch die ungemein wertvollen, praxisbezogenen MoMo-Befunde nicht einfach nur ergänzt oder vervollständigt, sondern diese liefern teilweise vollkommen neue Erkenntnisse über die Leistungsfähigkeit und Bewegungsaktivität von Kindern und Jugendlichen. Insbesondere in der Sportwissenschaft ist die reine Anwendung von Fragebögen zur Erhebung von Daten eher umstritten, da sie meistens praxisbezogene Untersuchungsgegenstände nicht realitätsnah abbilden können. Eine Unterfütterung solcher Daten durch Beobachtungs- oder Kohortenstudien wird deshalb meistens angeraten. Die bereits bestehenden Forschungsergebnisse deckten in diesem Fall zwar teilweise bis zu 27 Länder ab - Deutschland fand innerhalb dieser Studien allerdings nur wenig Beachtung und falls doch, so waren die erhobenen Daten nicht aussagekräftig genug (Albrecht, Hanssen-Doose, Bös, Schlenker, Schmidt, Wagner, Will \& Worth, 2016).

Wenn eine Erhebung gegenwärtiger und früherer Generationen zur motorisch-körperlichen Leistungsfähigkeit von Kindern und Jugendlichen durchgeführt wurde, so kann zum einen davon ausgegangen werden, dass derlei Beurteilungen und Bewertungen über motorische Kompetenzen häufig von Trainern/-innen und Lehrern/-innen vorgenommen wurden. Zum anderen ist aber eine derartige Ermittlung recht differenziert und komplex, da es laut Opper et al. (2007) bislang „keinen breiten Konsens über Tests, keine bundesweit gültigen Normierungen und keine repräsentativen Untersuchungen, die generalisierende Aussagen zur Motorik von Kindern und Jugendlichen erlauben“ (S. 879) gibt.

Opper et al. (2007) bemerken außerdem, dass „[a]uch Aussagen über das Ausmaß des Verlusts der motorischen Leistungsfähigkeit gegenüber früheren Generationen [ ] bisher aufgrund der zum Teil unterschiedlichen Erhebungsmethodik früherer und aktueller Studien ebenso wenig valide getroffen werden [konnten] wie differenzielle Aussagen bezüglich leistungsbeeinflussender Merkmale (Alter, Geschlecht, Sozialisationsmerkmale)“ (S. 879-880).

\subsection{Basiserhebung}

Die erste Basiserhebung fand von Juni 2003 bis Juni 2006 statt und wurde deutschlandweit an folgenden 167 Orten durchgeführt: 


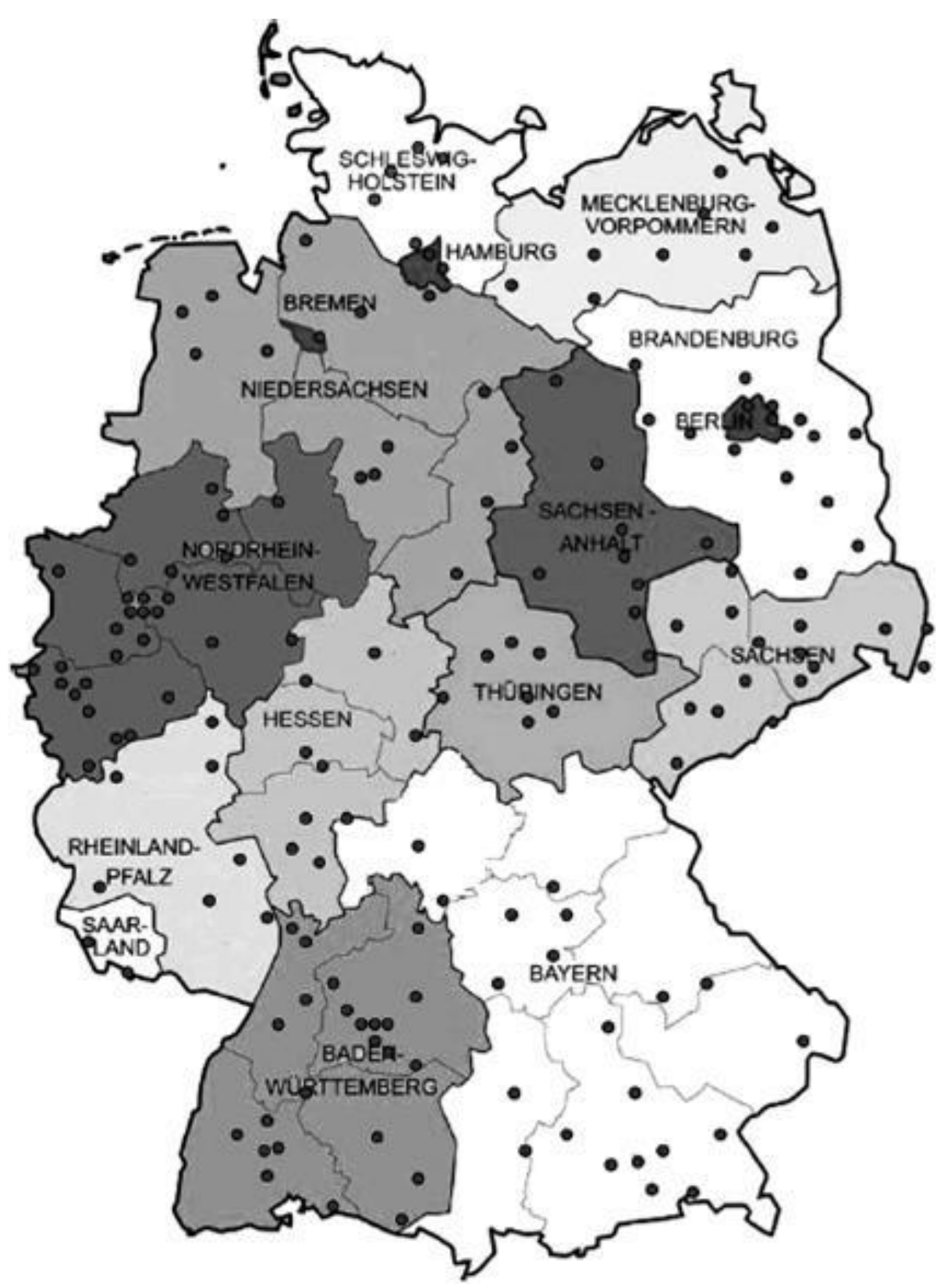

Abb. 3: Deutschlandkarte mit den insgesamt 167 Untersuchungsorten (Quelle: Opper et al., 2007, S. 885)

Hinsichtlich der Altersspanne von 4 bis 17 Jahren nahmen an dieser ersten Stichprobe 4.529 Mädchen und Jungen teil. Ein wichtiger Bestandteil der MoMo-Studie war wiederum ein Fragebogen, welcher den körperlich-sportlichen Aktivitätsgrad der Kinder und Jugendlichen messen sollte.

Zum anderen wurde jedoch ebenfalls eine praktische Erhebungsmethode durchgeführt, welche insgesamt 11 Testaufgaben (siehe Tab. 2) beinhaltet. Nach Bös (2001) lassen sich diese erneut untergliedern in folgende vier Testbereiche: „Koordination“, „Kraft“, „Beweglichkeit“ und „Ausdauerleistungsfähigkeit“ (S. 887), welche jedoch ausschließlich bei den 11- bis 17Jährigen getestet wurde. 


\begin{tabular}{|c|c|c|c|c|c|}
\hline \multirow[b]{2}{*}{ Testbereiche } & \multirow[b]{2}{*}{ Testinhalt } & \multirow[b]{2}{*}{ Testaufgaben } & \multicolumn{3}{|c|}{ Altersgruppen } \\
\hline & & & 4-5 Jahre & 6-10 Jahre & 11-17 Jahre \\
\hline \multirow[t]{5}{*}{ Koordination } & KP & Linie nachfahren (MLS) & $\mathrm{x}$ & $\mathrm{x}$ & $x$ \\
\hline & KP & Einbeinstand & $\mathrm{x}$ & $x$ & $\mathrm{x}$ \\
\hline & KP & Balancieren rückwärts & $\mathbf{x}$ & $\mathrm{x}$ & $x$ \\
\hline & KZ & Stifte einstecken (MLS) & $\mathrm{x}$ & $x$ & $x$ \\
\hline & RS & Reaktionstest & $\mathrm{x}$ & $\mathrm{x}$ & $\mathbf{x}$ \\
\hline \multirow[t]{4}{*}{ Kraft } & KA & Seitliches Hin- und Herspringen & $\mathrm{x}$ & $\mathrm{x}$ & $x$ \\
\hline & KA & Liegestütz & - & $\mathrm{X}$ & $x$ \\
\hline & SK & Standweitsprung & $\mathrm{x}$ & $x$ & $x$ \\
\hline & SK & Kraftmessplatte & $x$ & $x$ & $x$ \\
\hline Ausdauer & AA & Fahrradausdauertest & - & $x$ & $x$ \\
\hline Beweglichkeit & B & Rumpfbeugen & $\mathrm{x}$ & $\mathrm{x}$ & $x$ \\
\hline
\end{tabular}

Tab. 2: Erfassung der Motorik-Dimensionen im Kinder- und Jugendgesundheitssurvey (KiGGS) und Motorik-Modul (MoMo) (Quelle: Opper et al., 2007, S. 883)

Opper et al. (2007) fassen diese erste Basiserhebung innerhalb der MoMo-Studie wie folgt zusammen:

„Das erste Ziel des MoMo ist es, die Ist-Situation bezüglich motorischer Leistungsfähigkeit und körperlich-sportlicher Aktivität von Kindern und Jugendlichen zu analysieren. Auf Grundlage der repräsentativen Datenbasis wird damit eine Baseline geschaffen bzw. Normwerte für die Einordnung der motorischen Leistungsfähigkeit und des Sportverhaltens von Heranwachsenden festgelegt. Mit diesen Ausgangsdaten kann die motorische Entwicklung und das Sportverhalten zukünftig eingeordnet und in Beziehung gesetzt werden.

Damit ist die Basis für eine langfristige Aktivitäts- und Fitnessberichterstattung geschaffen. Ein weiteres wichtiges Ziel des MoMo ist die Analyse der Zusammenhänge zwischen körperlicher - und im Besonderen - sportlicher Aktivität, motorischer Leistungsfähigkeit und Gesundheit bzw. Gesundheitsverhalten. Diese Zusammenhänge können durch eine Verknüpfung mit den KiGGS-Daten erstmals belastbar analysiert werden. Weiterhin geht es auch darum, differenzierende Merkmale, wie z.B. den sozialen Status der Familie, den Migrationshintergrund und Unterschiede entlang der Wohnortgröße herauszuarbeiten. Die Ergebnisse der oben genannten Analyse liefern zudem eine wichtige empirische Grundlage, um Förderprogramme zur Bewegung und Aktivität in unterschiedlichen Settings, z. B. in Kindergärten, Schulen und Vereinen, entwickeln zu können." (S. 4) 


\subsection{KiGGS-Welle 1}

Die zweite Erhebung fand mit der sogenannten "KiGGS-Welle 1" von 2009 bis 2012 statt. Nun konnten diese Daten mit denjenigen aus der bereits erläuterten KiGGS-Basiserhebung (von 2003 bis 2006) gegenüber gestellt werden, wodurch eine erste repräsentative gesamtdeutsche Betrachtung dieses standardisierten Studiendesigns ermöglicht wurde. Bös (2003), der maßgeblich an dem MoMo-Studienkonzept beteiligt war, hatte schon im Jahr 2003 mit den Daten von 6- bis 17-Jährigen, welche im Zeitraum von 1965 bis 2002 untersucht wurden, eine Meta-Analyse von 54 Studien aus insgesamt 20 Ländern durchgeführt. Nachdem er die jeweiligen Daten zur motorischen Leistungsfähigkeit der Kinder und Jugendlichen miteinander verglichen hatte, kam er zu dem Ergebnis, dass im Zeitraum von 1965 bis 1975 eine Zunahme erkennbar ist, dann jedoch ab 1975 bis 2002 die Leistung von motorischen Fähigkeiten im Kindes- und Jugendalter um ca. 10 \% abnahm (Bös, 2003). An dieser Stelle muss hinzugefügt werden, dass derartige Erhebungen nie auf standardisierte Methoden fundierten, sondern hierbei „zumeist auf Ad-hoc-Stichproben [...] zurückgegriffen“ wurde (Albrecht et al., S. 296, 2016).

Eine Gegenüberstellung der Ergebnisse der Basiserhebung sowie der KiGGS-Welle 1 soll mögliche Veränderungen bzgl. der motorischen Leistungsfähigkeit von 4- bis 17-Jährigen aufdecken. In diesem Zusammenhang werden die Körperkonstitution, die körperliche Aktivität und der sozioökonomische Status der Probanden berücksichtigt, da diese Parameter die motorische Leistungsfähigkeit in vorherigen Studien am stärksten beeinflusst hat (Bös et al., 2009). Die folgende Tabelle gibt einen Überblick, inwiefern die Ergebnisse der Basiserhebung (2003 bis 2006) und der sogenannten Welle 1 (2009 bis 2012) hinsichtlich der eben genannten Beeinflussungsfaktoren zur motorischen Leistungsfähigkeit voneinander abweichen:

\begin{tabular}{|c|c|c|c|c|c|c|c|c|c|}
\hline & & \multicolumn{2}{|c|}{ 4-5 Jahre } & \multicolumn{2}{|c|}{ 6-10 Jahre } & \multicolumn{2}{|c|}{ 11-13 Jahre } & \multicolumn{2}{|c|}{ 14-17 Jahre } \\
\hline & & Basis & Welle 1 & Basis & Welle 1 & Basis & Welle 1 & Basis & Welle 1 \\
\hline \multirow{3}{*}{$\begin{array}{l}\text { BMI nach } \\
\text { Kromeyer- } \\
\text { Hauschild }^{a}\end{array}$} & Normalgewicht (\%) & 85,5 & 88,8 & 77,3 & 75,5 & 75,7 & 75,7 & 78,7 & 71,5 \\
\hline & Übergewicht (\%) & 8,3 & 7,4 & 14,6 & 17,1 & 16,3 & 18,9 & 15,2 & 19,7 \\
\hline & Untergewicht (\%) & 6,2 & 3,8 & 8,1 & 7,4 & 8,0 & 5,4 & 6,1 & 8,8 \\
\hline Größe (cm) & $\mathrm{MW}( \pm \mathrm{SD})$ & $\begin{array}{l}111,22 \\
( \pm 5,94)\end{array}$ & $\begin{array}{l}110,69 \\
( \pm 5,98)\end{array}$ & $\begin{array}{l}132,47 \\
( \pm 10,18)\end{array}$ & $\begin{array}{l}133,29 \\
( \pm 10,69)\end{array}$ & $\begin{array}{l}156,34 \\
( \pm 9,04)\end{array}$ & $\begin{array}{l}156,66 \\
( \pm 9,23)\end{array}$ & $\begin{array}{l}170,13 \\
( \pm 8,75)\end{array}$ & $\begin{array}{l}170,01 \\
( \pm 8,55)\end{array}$ \\
\hline \multirow{2}{*}{$\begin{array}{l}\text { Bewegungs- } \\
\text { empfehlung }^{b}\end{array}$} & Nicht erfüllt (\%) & 65,3 & 67,5 & 78,9 & 83,7 & 90,1 & 96,3 & 93,6 & 96,8 \\
\hline & Erfüllt (\%) & 34,7 & 32,5 & 21,1 & 16,3 & 9,9 & 3,7 & 6,4 & 3,2 \\
\hline \multirow{3}{*}{$\begin{array}{l}\text { Sportvereins- } \\
\text { mitglied }\end{array}$} & Ja $(\%)$ & 46,8 & 55,4 & 58,6 & 65,9 & 56,9 & 66,8 & 48,8 & 61,1 \\
\hline & Nein $(\%)$ & 47,8 & 39,2 & 30,1 & 21,0 & 20,2 & 13,9 & 19,1 & 11,3 \\
\hline & Früher $(\%)$ & 5,4 & 5,4 & 11,3 & 13,1 & 22,9 & 19,3 & 32,1 & 27,6 \\
\hline \multirow{3}{*}{$\begin{array}{l}\text { Sozioökono- } \\
\text { mischer Status } \\
\text { (SES) }\end{array}$} & Niedrig $(\%)$ & 23,4 & 8,7 & 19,7 & 17,2 & 22,5 & 22,6 & 16,2 & 22,6 \\
\hline & Mittel (\%) & 55,8 & 64,6 & 59,5 & 62,6 & 59,8 & 60,0 & 62,8 & 61,0 \\
\hline & $\operatorname{Hoch}(\%)$ & 20,8 & 26,8 & 20,8 & 20,2 & 17,7 & 17,4 & 21,0 & 16,4 \\
\hline
\end{tabular}


Laut Albrecht et al. (2016) können hieraus folgende Schlüsse gezogen werden:

Bewegungsempfehlung: Welle 1 ist durchweg um 2,2 - 6,2 \% geringer als die Basiserhebung.

Aktive Sportvereinstätigkeit: Welle 1 zeichnet einen Anstieg gegenüber der Basiserhebung auf.

BMI Übergewicht: Welle 1 weist einen Anstieg von durchweg 2,5 - 4,5 \% gegenüber der Basiserhebung auf.

(Niedriger) sozioökonomischer Status (SES): Welle 1 zeigt einen Rückgang um $2,5-14,7 \%$ gegenüber der Basiserhebung bei den 4- bis 5- bzw. 9- bis 10Jährigen, während sich die Werte bei den 14- bis 17-Jährigen um 6,4 \% erhöhen.

Die folgenden Tabellen bilden die konkreten Ergebnisse sowohl der Basis-, als auch der Welle-1-Erhebung hinsichtlich der durchgeführten Motorikstichproben aller männlichen und weiblichen Studienteilnehmer ab.

Zu den durchgeführten Bewegungsaufgaben zählten u.a. Standweitsprung, Rumpfbeugen und Balancieren rückwärts auf einem schmalen Balken:

\begin{tabular}{|c|c|c|c|c|c|c|c|c|c|}
\hline \multirow[t]{2}{*}{ Männlich } & & \multicolumn{2}{|c|}{ Altersgruppe 4-5 Jahre } & \multicolumn{2}{|c|}{ Altersgruppe 6-10 Jahre } & \multicolumn{2}{|c|}{ Altersgruppe 11-13 Jahre } & \multicolumn{2}{|c|}{ Altersgruppe 14-17 Jahre } \\
\hline & & Basis & Welle 1 & Basis & Welle 1 & Basis & Welle 1 & Basis & Welle 1 \\
\hline \multirow[t]{3}{*}{ Standweitsprung (cm) } & MW (95\% KI) & $91,4(88,5-94,2)$ & $\begin{array}{l}91,2 \\
(87,5-94,92)\end{array}$ & $\begin{array}{l}128,5 \\
(126,3-130,6)\end{array}$ & $\begin{array}{l}130,3 \\
(126,9-133,6)\end{array}$ & $\begin{array}{l}159,5 \\
(156,8-162,1)\end{array}$ & $\begin{array}{l}155,6 \\
(152,0-159,2)\end{array}$ & $\begin{array}{l}195,2 \\
(191,8-198,6)\end{array}$ & $\begin{array}{l}193,4 \\
(189,8-196,9)\end{array}$ \\
\hline & SD & 20,0 & 17,9 & 22,3 & 23,6 & 23,0 & 24,0 & 28,3 & 26,6 \\
\hline & Anzahl $(n)^{a}$ & 476 & 165 & 862 & 472 & 466 & 436 & 465 & 357 \\
\hline \multirow{3}{*}{$\begin{array}{l}\text { Seitliches Hin- und } \\
\text { Herspringen (Sprünge in } \\
15 \mathrm{~s} \text { ) }\end{array}$} & $M W(\mathrm{Kl})$ & $9,8(9,3-10,4)$ & $\begin{array}{l}11,9 \\
(11,2-12,6)\end{array}$ & $\begin{array}{l}19,8 \\
(19,1-20,6)\end{array}$ & $24,5(23,6-25,5)$ & $31,4(30,6-32,2)$ & $34,0(33,0-35,1)$ & $36(35,2-36,8)$ & $39,1(38,4-39,9)$ \\
\hline & SD & 4,0 & 3,7 & 6,6 & 73 & 6,5 & 6,6 & 7,0 & 6,0 \\
\hline & Anzahl $(n)^{n}$ & \multicolumn{2}{|l|}{468} & 852 & 471 & 462 & 436 & 466 & 357 \\
\hline \multirow[t]{3}{*}{ Liegestützen (in $40 \mathrm{~s}$ ) } & $\mathrm{MW}(\mathrm{KI})$ & \multicolumn{2}{|l|}{ Nicht erhoben } & $\begin{array}{l}10,1 \\
(9,8-10,5)\end{array}$ & $11,4(10,8-11,9)$ & $12,1(11,6-12,5)$ & $12,5(11,7-13,3)$ & $14,3(13,9-14,7)$ & $15,1(14,7-15,6)$ \\
\hline & SD & \multicolumn{2}{|l|}{ Nicht erhoben } & 3,7 & 36 & 3,4 & 4,4 & 4,0 & 3,6 \\
\hline & Anzahl $(n)^{\circ}$ & 0 & 0 & 852 & 492 & 466 & 359 & 464 & 352 \\
\hline \multirow[t]{3}{*}{$\begin{array}{l}\text { MLS Stifte einstecken } \\
\text { (Zeit in s) }\end{array}$} & $\mathrm{MW}(\mathrm{KI})$ & $\begin{array}{l}78,9 \\
(76,6-81,1)\end{array}$ & $75,7(73,4-78,0)$ & $\begin{array}{l}55,2 \\
(54,4-56,1)\end{array}$ & $53,8(52,7-54,9)$ & $46,9(46,1-47,4)$ & $44,7(43,9-45,5)$ & $43,2(42,7-43,8)$ & $42,0(41,4-42,6)$ \\
\hline & SD & 16,5 & 11,9 & 8,9 & 8,5 & 10,8 & 4,6 & 4,9 & 4,4 \\
\hline & Anzahl $(n)^{\prime \prime}$ & 477 & 165 & 853 & 474 & 455 & 437 & 458 & 361 \\
\hline \multirow{3}{*}{$\begin{array}{l}\text { Rumpfbeuge (cm über } \\
\text { und unter } \\
\text { Fußsohlenniveau) }\end{array}$} & $M W(\mathrm{KI})$ & $\begin{array}{l}-0,4 \\
(-1,1-0,4)\end{array}$ & $02(-0,9-1,3)$ & $\begin{array}{l}-2,1 \\
(-2,8--1,4)\end{array}$ & $0,00(-0,7-0,8)$ & $-3,1(-3,9-2,3)$ & $-3,4(-4,5--2,4)$ & $-1,9(-2,8--1,0)$ & $-2,2(-3,2-1,1)$ \\
\hline & SD & 6,3 & 4,9 & 6,8 & 6,1 & 6,8 & 7,2 & 8,6 & 8,7 \\
\hline & Anzahl $(n)^{\circ}$ & 458 & 165 & 850 & 474 & 464 & 437 & 467 & 361 \\
\hline \multirow{3}{*}{$\begin{array}{l}\text { Balancieren rückwärts } \\
\text { (Summe aus } \\
3 \text { Versuchen) }\end{array}$} & $M W(\mathrm{KI})$ & $\begin{array}{l}11,5 \\
(10,4-12,7)\end{array}$ & $14,4(12,5-16,3)$ & $\begin{array}{l}26,3 \\
(25,4-27,2)\end{array}$ & $28,7(27,3-30,2)$ & $32,3(31,2-33,5)$ & $35,3(34,1-36,6)$ & $33,4(32,3-34,5)$ & $37,3(36,3-38,3)$ \\
\hline & SD & 8,2 & 8,5 & 10,2 & 10,5 & 9,2 & 8,5 & 9,7 & 8,2 \\
\hline & Anzahl $(n)^{a}$ & 467 & 162 & 862 & 474 & 468 & 439 & 467 & 360 \\
\hline PWC (Watt/kg KG) & $M W(\mathrm{KI})$ & Nicht erhoben & & $\begin{array}{l}62,8 \\
(60,9-64,8)\end{array}$ & $68,8(66,3-71,4)$ & $\begin{array}{l}101,5 \\
(98,0-104,9)\end{array}$ & $\begin{array}{l}103,6 \\
(97,5-109,7)\end{array}$ & $\begin{array}{l}157,3 \\
(152,8-161,7)\end{array}$ & $\begin{array}{l}153,0 \\
(146,5-159,6)\end{array}$ \\
\hline & SD & Nicht erhoben & & 20,2 & 18,9 & 25,9 & 33,3 & 37,3 & 44,2 \\
\hline & Anzahl $(n)^{n}$ & 0 & & 739 & 345 & 413 & 358 & 410 & 309 \\
\hline
\end{tabular}

Tab. 4: Kohortenvergleich Motorikstichprobe; $n=2285$ zur Basiserhebung; $n=1441$ zur Welle 1; männliche Teilnehmer (Quelle: Albrecht et al., 2016, S. 300) 


\begin{tabular}{|c|c|c|c|c|c|c|c|c|c|}
\hline \multirow[t]{2}{*}{ Weiblich } & & \multicolumn{2}{|c|}{ Altersgruppe 4-5 Jahre } & \multicolumn{2}{|c|}{ Altersgruppe 6-10 Jahre } & \multicolumn{2}{|c|}{ Altersgruppe $11-13$ Jahre } & \multicolumn{2}{|c|}{ Altersgruppe 14-17 Jahre } \\
\hline & & Basis & Welle 1 & Basis & Welle 1 & Basis & Welle 1 & Basis & Welle 1 \\
\hline \multirow[t]{3}{*}{$\begin{array}{l}\text { Standweit- } \\
\text { sprung }(\mathrm{cm})\end{array}$} & MW (KI) & $\begin{array}{l}85,8 \\
(83,5-88,0)\end{array}$ & $\begin{array}{l}88,6 \\
(85,4-91,8)\end{array}$ & $\begin{array}{l}121,2 \\
(119,4-123,1)\end{array}$ & $\begin{array}{l}127,1 \\
(124,3-130,0)\end{array}$ & $\begin{array}{l}146,6 \\
(143,3-150,0)\end{array}$ & $\begin{array}{l}145,8 \\
(142,9-148,8)\end{array}$ & $\begin{array}{l}150,5 \\
(147,9-153,2)\end{array}$ & $\begin{array}{l}150,6 \\
(147,6-153,5)\end{array}$ \\
\hline & SD & 20,1 & 16,5 & 19,2 & 20,5 & 23,2 & 21,3 & 24,8 & 22,3 \\
\hline & $\begin{array}{l}\text { Anzahl } \\
(n)^{2}\end{array}$ & 501 & 166 & 847 & 510 & 413 & 424 & 471 & 356 \\
\hline \multirow{3}{*}{$\begin{array}{l}\text { Seitliches Hin- } \\
\text { und } \\
\text { Herspringen } \\
\text { (Sprünge in } \\
15 \text { s) }\end{array}$} & MW (KI) & $\begin{array}{l}10,3 \\
(9,8-10,7)\end{array}$ & $\begin{array}{l}12,8 \\
(11,8-13,8)\end{array}$ & $\begin{array}{l}21,3 \\
(20,6-22,0)\end{array}$ & $\begin{array}{l}26,2 \\
(25,1-27,2)\end{array}$ & $\begin{array}{l}32,5 \\
(31,8-33,2)\end{array}$ & $\begin{array}{l}33,4 \\
(32,6-34,2)\end{array}$ & $\begin{array}{l}34,5 \\
(33,8-35,1)\end{array}$ & $\begin{array}{l}36,0 \\
(35,1-36,9)\end{array}$ \\
\hline & SD & 3,5 & 4,4 & 6,7 & 7,1 & 5,7 & 5,8 & 5,8 & 6,5 \\
\hline & $\begin{array}{l}\text { Anzahl } \\
(n)^{2}\end{array}$ & 497 & 164 & 839 & 513 & 410 & 424 & 469 & 361 \\
\hline \multirow[t]{3}{*}{$\begin{array}{l}\text { Liegestützen } \\
\text { (in } 40 \text { s) }\end{array}$} & MW (KI) & \multicolumn{2}{|c|}{ Nicht erhoben } & $\begin{array}{l}10,0 \\
(9,6-10,4)\end{array}$ & $\begin{array}{l}11,4 \\
(10,9-12,0)\end{array}$ & $\begin{array}{l}11,7 \\
(11,1-12,2)\end{array}$ & $\begin{array}{l}12,3 \\
(11,8-12,8)\end{array}$ & $\begin{array}{l}12,1 \\
(11,6-12,5)\end{array}$ & $\begin{array}{l}12,3 \\
(11,9-12,7)\end{array}$ \\
\hline & SD & \multicolumn{2}{|c|}{ Nicht erhoben } & 3,5 & 3,6 & 3,5 & 3,6 & 3,6 & 3,2 \\
\hline & $\begin{array}{l}\text { Anzahl } \\
(n)^{3}\end{array}$ & 0 & 0 & 840 & 496 & 411 & 356 & 466 & 357 \\
\hline \multirow{3}{*}{$\begin{array}{l}\text { MLS Stifte } \\
\text { einstecken } \\
\text { (Zeit in s) }\end{array}$} & MW (KI) & $\begin{array}{l}73,4 \\
(71,9-75,0)\end{array}$ & $\begin{array}{l}71,6 \\
(69,2-74,1)\end{array}$ & $\begin{array}{l}52,4 \\
(51,6-53,2)\end{array}$ & $\begin{array}{l}50,8 \\
(49,6-51,9)\end{array}$ & $\begin{array}{l}44,1 \\
(43,5-44,7)\end{array}$ & $\begin{array}{l}43,7 \\
(42,9-44,5)\end{array}$ & $\begin{array}{l}41,7 \\
(41,2-41,2)\end{array}$ & $\begin{array}{l}40,4 \\
(39,9-40,9)\end{array}$ \\
\hline & SD & 12,9 & 11,8 & 8,2 & 8,2 & 4,8 & 5,6 & 4,5 & 3,9 \\
\hline & $\begin{array}{l}\text { Anzahl } \\
(n)^{2}\end{array}$ & 497 & 165 & 842 & 512 & 401 & 427 & 458 & 363 \\
\hline \multirow{3}{*}{$\begin{array}{l}\text { Rumpfbeuge } \\
\text { ( } \mathrm{cm} \text { über und } \\
\text { unter Fußsoh- } \\
\text { lenniveau) }\end{array}$} & MW (KI) & $\begin{array}{l}2,3 \\
(1,6-2,9)\end{array}$ & $\begin{array}{l}3,3 \\
(2,5-4,1)\end{array}$ & $1,1(0,4-1,7)$ & $3,5(2,7-4,3)$ & $\begin{array}{l}0,5 \\
(-0,5-1,5)\end{array}$ & $3,1(2,0-4,2)$ & $2,5(1,4-3,6)$ & $4,5(3,5-5,5)$ \\
\hline & SD & 5,4 & 3,8 & 6,5 & 6,2 & 7,8 & 8,2 & 9,4 & 8,4 \\
\hline & $\begin{array}{l}\text { Anzahl } \\
(n)^{2}\end{array}$ & 491 & 165 & 839 & 513 & 413 & 427 & 471 & 362 \\
\hline \multirow{3}{*}{$\begin{array}{l}\text { Balancieren } \\
\text { rückwärts } \\
\text { (Summe aus } 3 \\
\text { Versuchen) }\end{array}$} & MW (KI) & $\begin{array}{l}13,8 \\
(12,8-14,9)\end{array}$ & $\begin{array}{l}17,1 \\
(15,0-19,2)\end{array}$ & $\begin{array}{l}28,4 \\
(27,4-29,5)\end{array}$ & $\begin{array}{l}32,1 \\
(30,8-33,4)\end{array}$ & $\begin{array}{l}33,7 \\
(32,4-35,0)\end{array}$ & $\begin{array}{l}38,4 \\
(37,3-39,4)\end{array}$ & $\begin{array}{l}35,1 \\
(34,2-35,9)\end{array}$ & $\begin{array}{l}38,3 \\
(37,2-39,4)\end{array}$ \\
\hline & SD & 8,7 & 8,9 & 9,9 & 10,0 & 9,6 & 7,5 & 8,6 & 8,2 \\
\hline & $\begin{array}{l}\text { Anzahl } \\
(n)^{2}\end{array}$ & 497 & 163 & 848 & 512 & 414 & 426 & 472 & 363 \\
\hline \multirow[t]{3}{*}{$\begin{array}{l}\text { PWC (Watt/kg } \\
\text { KG) }\end{array}$} & MW (KI) & \multicolumn{2}{|c|}{ Nicht erhoben } & $\begin{array}{l}52,6 \\
(50,8-54,4)\end{array}$ & $\begin{array}{l}58,3 \\
(55,3-61,3)\end{array}$ & $\begin{array}{l}89,5 \\
(85,8-93,2)\end{array}$ & $\begin{array}{l}91,1 \\
(86,3-95,8)\end{array}$ & $\begin{array}{l}108,2 \\
(104,4-112,1)\end{array}$ & $\begin{array}{l}101,8 \\
(97,8-105,7)\end{array}$ \\
\hline & SD & \multicolumn{2}{|c|}{ Nicht erhoben } & 17,2 & 18,1 & 23,0 & 27,8 & 26,9 & 27,4 \\
\hline & $\begin{array}{l}\text { Anzahl } \\
(n)^{2}\end{array}$ & 0 & 0 & 723 & 375 & 379 & 352 & 423 & 294 \\
\hline
\end{tabular}

Tab. 5: Kohortenvergleich Motorikstichprobe; n=2243 zur Basiserhebung; n=1379 zur Welle 1; weibliche Teilnehmer (Quelle: Albrecht et al., 2016, S. 301)

Werden die Veränderungen der motorischen Leistungsfähigkeiten isoliert betrachtet, so kann anhand der beiden oberen Tabellen 4 und 5, welche die Werte der männlichen sowie weiblichen Probanden dieser Motorikstichprobe abbilden, folgendes ermittelt werden:

\section{Geschlechtsspezifische Unterschiede: Gering.}

Altersspezifisch: Positive Leistungsveränderung bei den 6- bis 10-jährigen Grundschulkindern in der Kohorte der Welle 1 gegenüber der Basiserhebung.

Konditionelle Fähigkeiten: Diese werden teilweise von beiden Geschlechtern in der Kohorte der Welle 1 mit besseren Ergebnissen als in der Kohorte der Basiserhebung erzielt, insbesondere wie erwähnt bei den 6- bis 10-Jährigen. Bei der Bewegungsaufgabe „Hin- und Herspringen“ werden innerhalb von 15 Sekunden teilweise 2 bis 5 mehr Sprünge in der Kohorte der Welle 1 erzielt. Auch bei den Bewegungsaufgaben „Liegestützen“ und „Fahrradausdauertest" sind die Ergebnisse in der Kohorte der Welle 1 besser. 
Großmotorische Koordination: Hinsichtlich dieses motorischen Teilbereichs leisten vor allem aus der Kohorte der Welle 1 die 11- bis 13-jährigen Mädchen neben der Bewegungsaufgabe „Seitliches Hin- und Herspringen“ beim „Balancieren rückwärts" bis zu 5 Schritte mehr als die Kohorte der Basiserhebung. Dies bedeutet, dass insgesamt betrachtet innerhalb dieses Bereichs bei den Mädchen eine Verbesserung von 8 bis $24 \%$ und bei den Jungen eine Verbesserung von 3 bis 24\% im Vergleich zur Basiserhebung konstituiert werden kann.

Feinmotorische Koordination: Hier ist keine signifikante Veränderung eingetreten. Der Wert liegt bspw. In der Kohorte der Welle 1 bei der Bewegungsaufgabe „MLS Stifte einstecken“ bei beiden Geschlechtern der 14- bis 17-Jährigen bei einer minimalen Leistungssteigerung von 1,25 Sekunden.

\subsection{Fazit und Diskussion}

Mit der Gegenüberstellung der erhobenen Daten aus den Jahren 2003 bis 2006 (Basiserhebung) und den Jahren 2009 bis 2012 (Welle 1) sollte erstmalig der Versuch unternommen werden, die Entwicklungen und Tendenzen der motorischen Leistungsfähigkeit von Kindern und Jugendlichen in Deutschland zu beschreiben und zu interpretieren. Bemerkbar wurde, dass bei der Längsschnittstudie teils stagnierende, aber auch insbesondere innerhalb der großmotorischen Koordinationstests verbesserte Ergebnisse der Welle-1-Kohorte auftraten. Schmidt, Will und Woll (2016) und Will, Schmidt und Woll (2016) bestätigen diesen Trend. Unter Berücksichtigung der MoMo-Studie wurden durch die getätigten Umfragen ersichtlich, dass neben dem Anstieg der wöchentlichen körperlich-sportlichen Stundenzahl um ca. 6,5 \% „sich die Kinder und Jugendlichen vermehrt dem Sporttreiben in Schul-AGs und im Sportverein zu[wenden]“ (Albrecht et al., 2016, S. 299). Darüber hinaus ist „[d]as leicht gesteigerte Ausmaß an organisiertem Sport [ ] möglicherweise als ein Einflussfaktor für die positive Entwicklungsrichtung von Basiserhebung und Welle 1 zu sehen" (ebd.).

Obgleich ein positiver Trend aufgrund der Ergebnisse der Welle 1 zu verzeichnen ist, so wird die Bewegungsempfehlung von 60 Minuten pro Tag bei mittlerer bis höherer Intensität dennoch von der Mehrzahl deutscher Kinder und Jugendlicher nicht erreicht. Daher sollte weiter untersucht werden, ob eventuell ein Zusammenhang zwischen der erhöhten Aktivität im organisiertem Sport und dem reduzierten Aktivitätsgrad im Alltag besteht (Albrecht et al., 2016). Ebenfalls interessant ist, dass sich in der Welle $1 \mathrm{im}$ Vergleich zur Basiserhebung ein 
geringerer Anteil an Teilnehmern befindet, welche aus sozioökonomisch niedrigen, ergo sozialschwächeren Familien kommen. Diese Gegebenheit könnte ebenfalls für eine Verbesserung der Ergebnisse hinsichtlich der Leistungsfähigkeit deutscher Kinder und Jugendlicher sprechen, da sich in früheren Studien oftmals ein kongruentes Verhältnis zwischen niedrigem Sozialstatus und schwachen motorischen Leistungsfähigkeiten zeigte (Bös et al., 2009). Nichtsdestotrotz sollte der sozioökonomische Status als eine Variable und nicht als Normative gesehen werden. Auch in diesem Fall wären weitere Studien hilfreich (Klein, Fröhlich \& Emrich, 2011).

Hinsichtlich der besseren Resultate der Kinder im Grundschulalter im Gegensatz zu den Ergebnissen der Jugendlichen wird jedoch stark vermutet, dass Grundschulkinder häufig eine bessere Förderung ihres Bewegungsdrangs erfahren. An weiterführenden Schulen ist laut Bös et al. (2008) diesbezüglich das Gegenteil der Fall. Doch auch aufgrund des Reifungsprozesses sind Grundschulkinder in der sportmotorischen, koordinativen Entwicklung deutlich aufnahmekompetenter als pubertierende Jugendliche, was die ebenfalls deutlich besseren Werte in der Bewegungsaufgabe „Hin- und Herspringen“ zusätzlich unterstreichen. Diese Übung wird mitunter sehr von der Koordinationsfähigkeit bestimmt (Albrecht et al., 2016). Pubertierende stecken oftmals in einer sogenannten „Koordinationskrise“ (Dickhuth, 2000), da ihnen das Koordinieren der plötzlich wachsenden Extremitäten (Arme und Beine) extrem schwer fällt. Mit Hilfe dieser Erkenntnisse können in besonderem Maße weitere zielgerichtete Fördermaßnahmen von Bewegung in Grundschulen bestimmt werden, damit diese leicht positive Tendenz beibehalten und zukünftig weiter gestärkt wird. In Anbetracht der Resultate muss darüber hinaus insbesondere in weiterführenden Schulen ein Ausbaukonzept für Bewegungsförderung eruiert werden, sodass auch dort eine positive Entwicklung im höheren Schulalter umgesetzt wird (Albrecht et al., 2016).

Durch die Erhebungen der KiGGS- und MoMo-Studien ist es gelungen, ausfindig zu machen, wer u.a. die hauptsächlich Betroffenen des konstituierten Bewegungsmangels sind. Anhand der gelieferten Statistiken sind Kinder und Jugendliche mit Migrationshintergrund und sozial schwächer gestellte, diejenigen, die unbedingt zeitnah eine Förderung und Unterstützung benötigen. Wie schon angeklungen ist, werden Schulen und Vereine dazu aufgerufen, ihren Beitrag auszuweiten. Wie soll das aber vor allem in Halbtagsschulen passieren, wenn nicht mehr Sportstunden zur Verfügung stehen?

Genau hier könnte die Ganztagsschule ansetzen. Denn die Ganztagsschule hat aufgrund des größeren Zeitkontingents die Möglichkeit, die Zeitfenster mit Spiel, Sport und Bewegung zu 
füllen und folglich einen erheblichen Mehrwert zu leisten. Zudem könnte das Ganztagsschulkonzept diejenigen SuS erreichen, die aus finanziellen oder kulturellen Gründen den Vereinssport nicht aufsuchen können. Innerhalb einer Ganztagsschule kann ein Setting geboten werden, in dem Bildung und Erziehung durch und zum Sport verstärkt stattfände. Somit könnten nicht nur Bewegungsdefizite deutscher SuS eingeschränkt, sondern ebenfalls ein aktiv gesunder Lebensstil gefördert werden.

\section{Praktische Umsetzungsmöglichkeiten des Sports in der Ganztagsschule}

Warum Bewegung insbesondere für Kinder und Jugendliche dermaßen wichtig ist, wurde anhand der vorgestellten KiGGS- und MoMo-Studien (vgl. u.a. Bös, 2003; Lampert et al., 2007; Opper et al., 2007) ausgiebig erörtert. Der erhebliche Rückgang des körperlichen und sportlichen Aktivitätsgrads von Kindern und Jugendlichen wird in der aktuellen Forschung durchaus kritisch angemahnt. Doch welche Möglichkeiten hat der Sport innerhalb der Ganztagsschule, um dieser Problematik effizient entgegenzutreten? Und welche Kooperationsmodelle könnten den Schulsport in seiner Funktion entlasten? Im Folgenden sollen einige praktische Umsetzungsmöglichkeiten vorgestellt werden. Neben den beiden additiven Modellen „Sport am Nachmittag“ und „Sport im Ganztag“ sowie dem integrativen Modell der „Bewegten Schule“ soll auch das Basketball-Modell der Stadt Tübingen veranschaulicht werden.

Davor wird die Studie „1000 gelebte Kindertage“ von Kleine (2003), welche der erwähnten Studienlage bzgl. des Aktivitätsgrad von Kindern und Jugendlichen eher kritisch gegenübersteht sowie Lagings mehrdimensionales und anthropologisch fundiertes Bewegungsverständnis erörtert.

\subsection{Studie „1000 gelebte Kindertage“ von Kleine (2003)}

Laging (2006) vertritt im Gegensatz zu den Studien KiGGS und MoMo bzgl. der diskutierten Problematik, Kinder und Jugendliche würden sich zu wenig bewegen, eine konträre Meinung, in dem er entgegen der erwähnten Studienlage annimmt, dass Bewegung für Kinder und Jugendliche immer noch die wichtigste Aktivität überhaupt sei. Er nennt als unterstützenden Beweis seiner Aussagen die Studie „1000 gelebte Kindertage“ von Kleine (2003), wonach Kinder an 98 \% der untersuchten 1000 Tage in Bewegung waren. Die Gesamtbewegungszeit aller Mädchen und Jungen, die an dieser Studie teilgenommen haben, lag bei 
durchschnittlich 3,5 Stunden pro Tag. Diese Zahlen widersprechen beispielweise der Studienergebnisse von Bös (2003) enorm. Diese besagen, dass sich Kinder angeblich nur eine Stunde pro Tag bewegen würden.

Laging (2006) und Kleine (2003) kritisieren folglich die Defizithypothese „Kinder bewegen sich zu wenig" vehement. Sie glauben, dass sich Kinder vor allem in informellen Situationen im Alltag mit Bewegung, Spiel und Sport neben den vielfältigen neuen Medien ausreichend befassen. Ergo prallen diesbezüglich zwei völlig konträre Studienergebnisse aufeinander, was eine etwas undurchsichtige Studienlage suggeriert.

\subsection{Das anthropologische Bewegungsverständnis Lagings}

Doch wie kann begründet werden, dass Kinder sich gerne bewegen und in welcher Art und Weise tun sie dies? Laging (2006) beobachtete, dass sich Kinder neben dem instrumentell geschickten Gebrauch ihrer Körper, ebenfalls mit Bezug auf die soziale und materiale Welt bewegen. Das bedeutet, dass der Interaktion und dem Zusammen- und Miteinanderspielen von Kindern ein erheblicher Stellenwert zugeschrieben werden kann:

„Die Bewegungsaktivitäten sind in ein Handlungsgeschehen eingebunden, das mehr bedeutet als eine äußerlich beobachtbare Motorik oder eine messbare Fitness. Es geht um ein intentionales und sinnbesetztes Bewegungshandeln, das nur der Mensch selbst hervorbringen kann. [...] Jede Bewegung ist unmittelbar spürbar, kaum eine andere Handlung lässt das ,Ich' und das ,Andere' sowie die Relation zwischen Ich und Welt so direkt aufmerken“ (Laging, 2006, S. 2).

Laging (2006) vertritt ergo ein durchaus mehrdimensionales, philosophisches und anthropologisches Verständnis von Bewegung und körperlicher Aktivität, das sich von einer rein physiologisch basierten und körper- bzw. gesundheitsbezogenen Ebene deutlich abgrenzt. Er ist sich sicher, dass Kinder und Jugendliche in der Bewegung etwas über sich und ihre Möglichkeiten lernen. Laging möchte mit diesem Bewegungsverständnis verdeutlichen, „dass Kinder viele Gelegenheiten brauchen, um sich in ihrem Lebensalltag bewegen zu können“ (2006, S. 2).

Da die Schule derjenige Ort ist, an dem Kinder und Jugendliche den Großteil ihres Tages verbringen, sollte dieser Ort selbstverständlich ausreichend Bewegungsräume bieten. Festzuhalten bleibt, dass der Schulraum für bewegungsbezogene Bildungsprozesse genutzt werden 
sollte und es hierfür auch zahlreiche praktische Umsetzungsmöglichkeiten gibt. Gerade in dem Konzept einer Ganztagsschule ist darüber hinaus das größere Zeitbudget eine große Hilfe bei der Umsetzung solcher Prozesse.

Laging (2006) nennt in diesem Zusammenhang drei sogenannte „Entwicklungslinien“ bzw. Modelle, die an deutschen Schulen entstanden sind und die bestmöglich vernetzt werden müssen: Sportangebote in der außerunterrichtlichen Nachmittagsgestaltung, das pädagogische Konzept „Sport im Ganztag“ der Landessportbünde und die „Bewegte Schule“ als umfassendes integratives Bildungsangebot.

\subsection{Kooperationen von Schule und Sport - ein Beitrag zur Schulkultur?}

Fessler und Ziroli (1997) stellen mit Bezug auf die Zusammenarbeit von Schule und Verein fest, dass die Vernetzung und das Zusammenwirken unterschiedlicher Systeme in allen gesellschaftlichen Bereichen an Bedeutung zunehmen: „Dies gilt auch für die Kooperation der Schule mit Vereinen [...]“ (S. 9). Während der Bildungsreform von 1968 bis etwa 1975 öffnete sich die Schule zu ihrem sozialen Umfeld. Zunächst wurden im Jahre 1985 Kooperationsprogramme in Nordrhein-Westfalen und 1987 in Baden-Württemberg eingerichtet. Im Zeitraum von 1992 bis 1996 fügten die restlichen Bundesländer Kooperationen mit Sportvereinen ein. Das Verhältnis des Miteinanders von Schule und Sportverein möchte das Ziel eines „Lebensraumes“ für SuS verwirklichen, damit sich die Kinder und Jugendlichen in der Schule wohl fühlen und Leben und Lernen in einem betrachtet wird (Fessler \& Ziroli, 1997).

Im folgenden Abschnitt werden die bereits genannten Modelle der Kooperation von Schule und Sport von Laging und Hildebrandt-Stramann (2006) erklärt. Darüber hinaus sollen sie hinsichtlich ihres Beitrags zur Entwicklung von Ganztagsschulen und zur Schulkultur reflektiert werden. Interessenskonflikte der Kooperationspartner Schule und Sportverein werden ebenfalls zusammengefasst und diskutiert. Zu guter Letzt wird das Basketball-Modell der Stadt Tübingen vorgestellt.

\subsection{Sport am Nachmittag - das additiv-duale Kooperationsmodell}

Aus pragmatischen Gründen weisen viele Ganztagsschulen in Deutschland per se einen hohen Anteil an außerunterrichtlichen Angeboten aus. Dazu gehören oft vielzählige Spiel-, Sport- und Bewegungsaktivitäten. Insbesondere Ganztagsschulen mit pädagogischer Mittagsbetreuung und offene Ganztagsschulen sind dazu in der Lage, Sportangebote in das 
Schulprogramm aufzunehmen (Holtappels, 2005). Diese Angebote finden häufig in Kooperation mit Partnern, wie bspw. dem Sportverein, statt und beruhen auf einer freiwilligen Teilnahme.

Die Tradition der Kooperation von Schule und Verein, die unabhängig von der Ganztagsschule existiert, sorgt für ein abwechslungsreiches, außerunterrichtliches Sportangebot. Sogenannte Landeskooperationsprogramme, welche in jedem Bundesland bestehen, sind zudem für die Förderung der Zusammenarbeit von Schulen und Sportvereinen zuständig. Ähnliche Untersuchungen zeigen außerdem, dass sich unabhängig von der jeweiligen Bildungslandschaft strukturell sehr ähnliche Programme entwickelt und etabliert haben. Diese Kooperationen sollten jedoch in der heutigen Zeit durch die Ganztagsschulentwicklung neu überdacht und reformiert werden. Dies ist durch die Landeskooperationsprogramme auch bereits der Fall. Die Zusammenarbeit von Schulen und Sportvereinen sowie die Entwicklung und Etablierung von Programmen werden von den Ländern explizit gefördert (Laging \& HildebrandtStramann, 2006).

Natürlich ist das Zustandekommen der Sportangebote sehr stark von den personalen Ressourcen, also den Sportlehrerinnen und Sportlehrern der einzelnen Schulen abhängig. Diese können bspw. selbst in Sportvereinen tätig sein und dort persönliche Kontakte zu den Übungsleitern herstellen. Eine enge Kooperation zwischen Verein und Schule ist in jedem Fall für beide Seiten gewinnbringend. Talentsichtungs- und Fördermaßnahmen leistungssportorientierter SuS können so besonders erfolgreich ablaufen, aber auch der Breitensport wird durch die von der Schule an die Sportvereine weitergeleiteten SuS gestärkt. So fasst Laging (2006) zusammen, dass „[d]ie Entscheidung für das eine oder andere Angebot [ ] also meist auf gewachsene Strukturen oder persönliches Engagement Einzelner zurück“ (S. 3) geht.

Insgesamt handelt es sich bei diesem Modell um ein additives Kooperationsmodell, das einer gemeinsamen Ganztagsbildung nicht verpflichtet ist. Ergo stehen Vormittag und Nachmittag unverbunden nebeneinander.

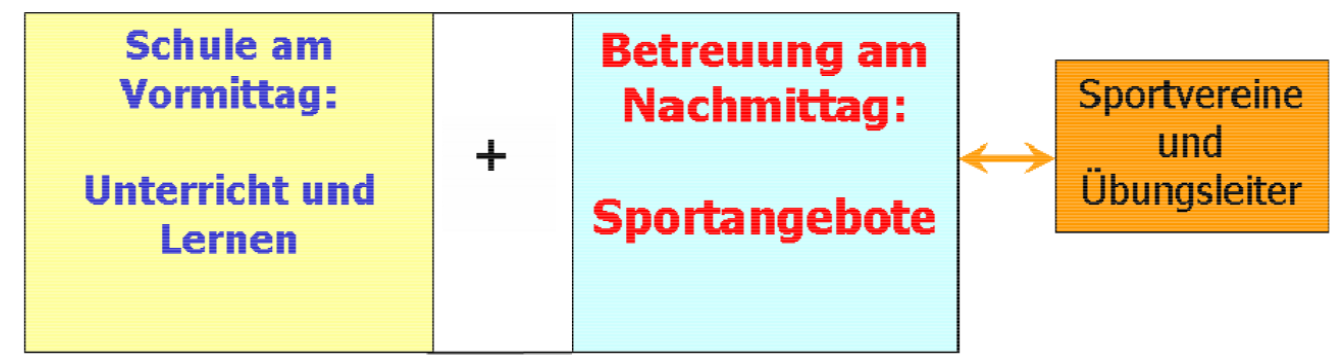

Abb. 4: Sport am Nachmittag - das additiv-duale Kooperationsmodell (Laging \& Hildebrandt-Stramann, 2006, S. 16) 


\subsection{Modell „Sport im Ganztag“ der Landessportbünde}

Einige Landessportbünde haben ein pädagogisches Konzept geschaffen, durch welches der gemeinnützig organisierte Sport nicht einfach nur den Leistungsgedanken verfolgen soll, sondern spezifische Bildungsabsichten besitzt und bspw. zielgerichtet die Erziehung und Persönlichkeitsentwicklung von Kindern und Jugendlichen fördert. So wurden Schwerpunkte in der Gesundheitsbildung, in der allgemeinen Freizeitpädagogik, Umweltbildung, interkulturellen Arbeit oder der Sicherheitserziehung im Straßenverkehr (Grabs, 2004) gesetzt. Das Programm „Sport im Ganztag“ entstand auf dieser Grundlage und richtet sich in erster Linie auf offene Ganztagsschulen. Die bewegungs- und sportorientierte Förderung Kinder und Jugendlicher steht hierbei im Vordergrund. In diesem Programm, dass sich zwischen dem Vereinssport und dem Schulsport positioniert, sollen neben den bekannten sportartbezogenen Angeboten auch offenere sportliche Aktivitäten, interkulturelle Spiel- und Bewegungsangebote, sogenannte Sportwochen und -feste sowie Talentsichtungsmaßnahmen durchgeführt werden (LSB NRW, 2005).

Dieses Konzept sollte als Erweiterung zum schulsportlichen Angebot verstanden werden. Durch das Konzept der Ganztagsschule könnte außerschulische Jugendarbeit und Schule einfacher miteinander verknüpft werden. Laging (2006) spricht von dem gleichberechtigten Nebeneinander von formeller schulischer Bildung und nicht-formeller Jugendbildung. Die Verlängerung des Schultags könnte jedoch auch zur Folge haben, dass die außerschulische Jugendarbeit zu kurz käme. Dies würde ebenfalls den organisierten Sport mit seiner Vereinsarbeit betreffen. Ein Konzept, dass die Verknüpfung von Schule und außerschulischer Jugendarbeit deutlich einfacher ermöglichen würde, ist unbestritten das der offenen Ganztagsschule. Nur in einem offenen Modell wären die Schulen in der Lage, den SuS ausreichend Zeit für außerschulische Vereinsaktivitäten zu gewährleisten. In einem gebundenen Konzept würde die eigene Gestaltung der Kinder- und Jugendlichen bezüglich ihrer Vereinsaktivitäten deutlich erschwert werden. Im optimalen Fall bilden die pädagogisch und bildungsorientierten Schulen und die kompetenz- und leistungsorientierten Vereine in Zukunft partnerschaftliche Arbeitszusammenhänge. So könnten Synergien geschaffen werden, von denen die SuS bzw. Kinder und Jugendlichen am meisten profitieren würden. Zudem bestünde so nicht mehr die Gefahr, dass sich die beiden Systeme wechselseitig kompensieren, belehren oder gar ersetzen wollen (Laging, 2006).

Laging (2006) spricht darüber hinaus dem „Sport im Ganztag“ die Empfehlung aus, sich nicht primär auf die Vermittlung von sportlichem Können oder die Kompensation von Bewe- 
gungsdefiziten zu versteifen, sondern insbesondere persönlichkeitsbildende Prozesse, Gemeinschaftsorientierung und die symbolische Reproduktion der Lebenswelt zu fördern. Grabs (2004) vertritt eine ähnliche Perspektive, geht jedoch spezifischer auf die außerschulische Jugendarbeit des Sports im Landessportbund NRW ein: „Das Spezifische an der Bildungsarbeit der Sportjugend besteht darin, dass Erlebnismöglichkeiten geschaffen werden, die in erster Linie Spaß machen, die freiwillig angenommen und mitgestaltet werden und das diese durch Reflexionsprozesse zu Erfahrungen werden können“ (S. 107).

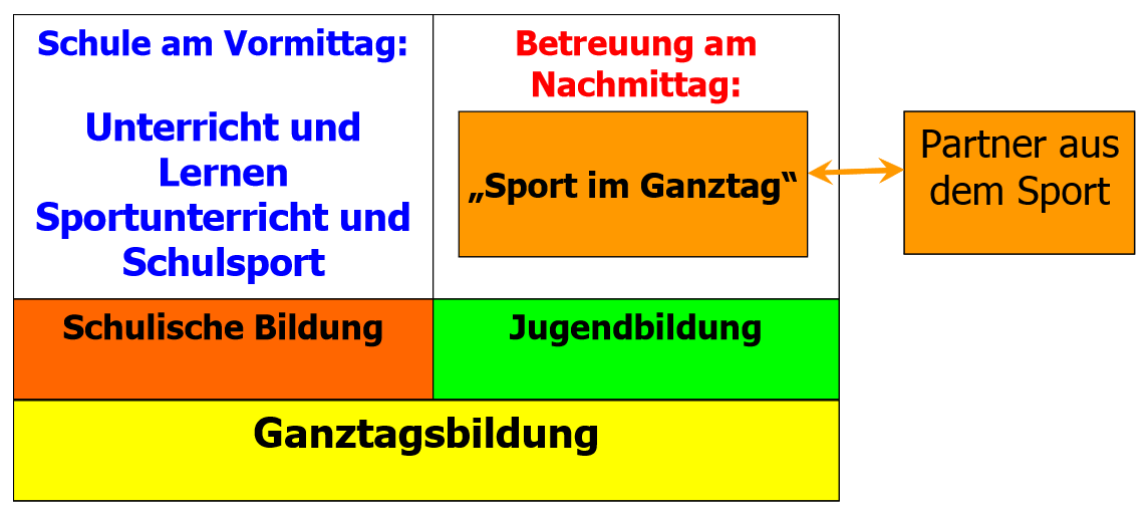

Abb. 5: Kooperation durch kompensatorische Ganztagsbildung - Modell „Sport im Ganztag“ (Laging \& HildebrandtStramann, 2006, S. 17)

Laging und Hildebrandt-Stramann (2006) befürchten, dass wenn die Entwicklung auf dieser Grundlage stehen bliebe, sich eher das arbeitsteilige Verfahren der Ganztagsbildung etablieren werde. Der Sport würde folglich für sich einen eigenen Teil der verfügbaren SuS-Zeit reklamieren und sich einem eigenen Bildungsanspruch verpflichten.

So wäre die weitere Entwicklung einer Schulkultur stark gefährdet, insofern sie sich mit weiteren Kulturen „überfrachten“ würde. Laging \& Hildebrandt-Stramann (2006) glauben, dass das Arbeitsbündnis zwischen Schule und Sport nur dann eine Chance hat, wenn diese „im Kontext eines gemeinsamen pädagogischen Systems mit Bildungsanspruch“ (S. 17) zusammenarbeiten und dieses Vorgehen sowohl vormittags, als auch nachmittags wechselseitig durchgezogen wird.

Nach Laging (2006) zeichnen sich die Sportvereine allerdings noch zu stark durch „pragmatisches Sporttreiben oder erlebnisträchtiges Machen aus“ (S. 5). Er räumt insbesondere dem Konzept der offenen Ganztagsschulen viele Möglichkeiten zu, wie bspw. die Entstehung einer „netzwerkartige[n] Kooperation zwischen eigenständigen Partnern“ (S. 6). So kann laut Laging (2006) das Bewegungsleben der Kinder und Jugendlichen enorm gefördert werden. 


\subsection{Das Modell der Bewegten Schule - Ein integrativer Ansatz}

Seit ca. 20 bis 25 Jahren ist die Bewegte Schule in Deutschland ein Ansatz, um Bewegung in den Schulalltag der Kinder und Jugendlichen zu bringen. Ihr Konzept geht auf den Schweizer Urs Illi zurück, welcher insbesondere das sedentäre Verhalten (zu langes Sitzen) der SuS kritisierte (vgl. Pühse \& Illi, 1999). Doch es gibt auch weitere Anlässe, welche die Relevanz von Bewegung in den deutschen Schulen verdeutlichen. Zum einen die wie schon in den erläuterten Studien KiGGS und MoMo thematisierte problematische Verringerung der körperlichen Aktivität durch den u.a. angestiegenen Medienkonsum und der damit einhergehende bedenkliche Gesundheitsstatus vieler Kinder und Jugendlicher, wie bspw. Adipositas, Diabetes oder Haltungsschäden. Zudem wurden neue Erkenntnisse aus der Lern- und Entwicklungsforschung zur Bedeutung von Bewegung (siehe Kapitel 5.2 über Kognitive Fähigkeiten) ernst genommen und die vorherrschende Bewegungsarmut an deutschen Schulen erkannt. Neben den sportpädagogischen Intentionen der Bewegten Schule, welche die körperliche Aktivität der SuS durch vermehrte Sport- und Spielangebote im Schulalltag forcieren soll, geht es diesem integrativen Ansatz auch darum, die innere Bewegung zu aktivieren, denn einer äußeren Bewegung geht eine innere voraus. Das System Schule kann nur in Bewegung gehalten werden, wenn alle an einem Strang ziehen: Lehrerinnen und Lehrer sowie SuS. Die Schule soll als Bewegungsraum erfahren werden. Die Schulräume sollten dementsprechend bewegungsfreundlich eingerichtet sein - sowohl die Lehrer- und die Klassenzimmer als auch der Pausenhof sollten dazu einladen, sich körperlich zu aktivieren. Wie eine solche Gestaltung der Räume einer ganztägig bewegten Schule aussehen könnte, wird im Kapitel „Praktische Umsetzungsmöglichkeiten - Die acht Bausteine“ dargelegt.

Unter der Berücksichtigung eines pädagogischen Arbeitsbündnisses unterschiedlicher Professionen sollten Kooperationen zwischen Lehrkräften und inkludierten außerschulischen Professionen wie bspw. Trainern/-innen oder Sportwissenschaftlern/-innen stattfinden. So würde die Schulkultur aus je unterschiedlichen Perspektiven und Aufgaben generiert werden, was folglich verstärkt positive Effekte auf diesen Prozess haben kann. Laging \& Hildebrandt-Stramann (2006) sind der Meinung, dass dies auf die Entwicklung einer bewegten Schulkultur übertragen heißen würde, dass „sich Bewegungs- und Sportlehrerinnen und lehrer gemeinsam mit anderen Professionen aus Bewegung, Spiel und Sport vor allem um die außerunterrichtlich-formellen, nicht-formellen bzw. informellen Bewegungs- und Sportgelegenheiten kümmern, möglicherweise bieten sich auch Kooperationen in der formellen Bildung des Sportunterrichts an" (S. 18). Hinsichtlich der Bewegungskompetenz ist die Ver- 
mittlungsleistung von Schule eine zweifache: Zum einen geht es laut Laging \& HildebrandtStramann (2006) um den fachlichen Lerngegenstand im Sinne von bewegungsbezogenen Aufgabenlösungen und zum anderen um Bewegung in einer Weltvermittlungsfunktion, Bewegung als grundlegende sozialleibliche Verständigung mit der Welt. Laut Helsper (2001) ginge es folglich darum, bewegungsbezogene Bildungsprozesse als öffnende Krisen „bisheriger Sichtweisen, Deutungen, Erklärungen oder Weltbilder von SchülerInnen“ (S. 29) wahrzunehmen, wodurch ein eindringlicher Blick auf das eigene Bewegen ersichtlich wird.

Derartige Bildungsprozesse sollten die eben genannten Professionen anstreben. Auch Laging \& Hildebrandt-Stramann (2006) glauben, dass sich erst daraus „eine andere Schulkultur, die den Begründungen für eine Ganztagsschule nahe kommt“ ergibt (S. 18) und es hier „nicht mehr um Betreuung, sondern um die Ermöglichung von Bildungsprozessen aus unterschiedlichen Perspektiven und unter Beteiligung von außerschulischen Professionen des Sports" gehe (S. 18-19). Die Kooperationspartner würden somit in das pädagogische System Schule mit ihren jeweils eigenen Zugängen zu Bewegung, Spiel und Sport integriert werden. Beide Kooperationspartner hätten gleichzeitig eine gemeinsame Verantwortung für eine Bewegungskompetenz im Interesse von bewegungsbezogener Selbstbildung und würden im Optimalfall nicht mehr als Einzelkämpfer egoistische Ziele verfolgen.

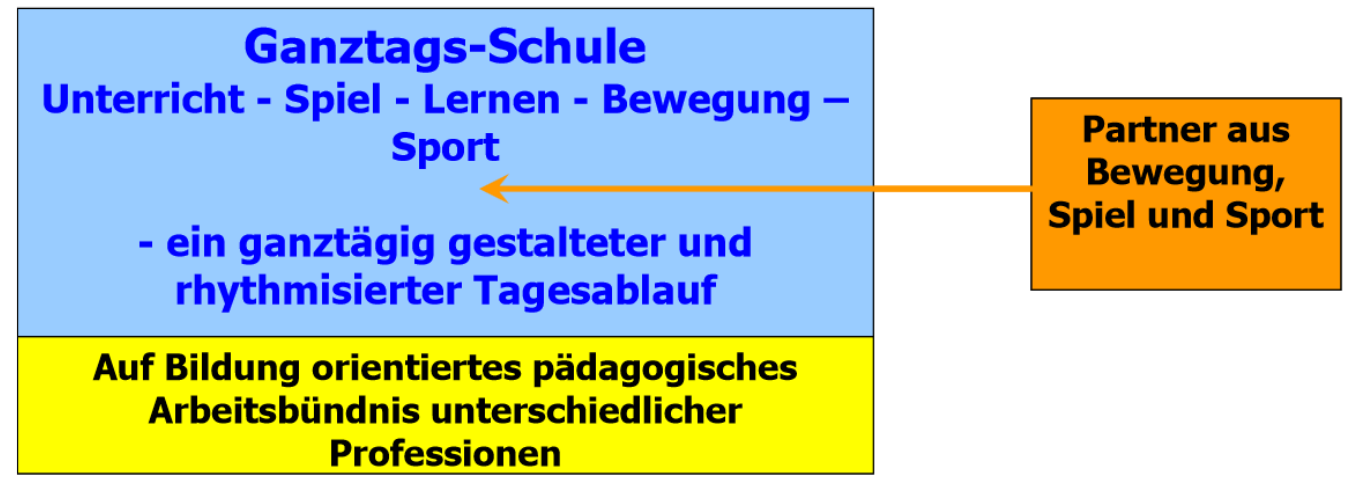

Abb. 6: Modell „Kooperation durch Inklusion von Bewegung, Spiel und Sport (Laging \& Hildebrandt-Stramann, 2006, S. 19)

Ergo würde dieses Konzept weit über den Betreuungsansatz und die bereits vorgestellten additiven Modelle in der offenen Ganztagsschule gehen. Die Bewegte Schule verlangt vom organisierten Sport eine Art „Selbstreflexion der eigenen Möglichkeiten im pädagogischen System der Schule mit eindeutigem Bildungsanspruch“ (Laging \& Hildebrandt-Stramann, 2006, S. 19). 


\subsection{Fazit und Diskussion}

Die unterschiedlichen Wirkungen der drei vorgestellten Kooperationsmodelle auf die Schulkultur könnten nicht divergierender ausfallen. Während die additiven Modelle "Sport am Nachmittag“ und „Sport im Ganztag“ eher eine eigene Sportkultur neben der bereits bestehenden Schulkultur bei den SuS etablieren, kann in dem integrativen Modell der „Bewegten Schule" die Schulkultur durch außerschulische Partner gezielter mitgestaltet werden. Die Schule müsste aus pädagogischer Sicht als ein umfassender Bewegungsraum verstanden werden, in dem laut Laging \& Hildebrandt-Stramann (2006) „das Leistungsverständnis [ ] durch unterschiedliche Erfahrungen in formellen und nicht-formellen sowie informellen Situationen die Selbstwirksamkeit des Leistens zwischen Selbst- und Fremdwahrnehmung differenzieren“ (S. 19) kann, die jeweiligen schulischen Inhalte eine stärkere Öffnung zur Lebenswelt zulassen, kulturelle Hintergründe eine Chance bekommen und „die Schulkultur eine größere Partizipation durch die aktive Beteiligung von SuS an der Gestaltung einer bewegungsorientierten Schule und durch die gleichberechtigte Kooperation unterschiedlicher Professionen“ (ebd.) erfährt. Falls diese Gegebenheiten eintreten sollten, dann wäre eine Bewegte Schule mit Vorbildcharakter geboren.

\subsection{Interessenskonflikte der Kooperationspartner Schule und Sportverein}

Wie bereits angeklungen ist, benötigt die Schule den Sport bei der Umsetzung eines Ganztagsschulkonzepts dringend, um nachmittägliche Betreuungsangebote anbieten zu können. Der Interessenkonflikt zwischen Schule und Verein ist jedoch in den meisten Fällen zu groß. So kann laut Laging und Hildebrandt-Stramann (2006) „kein professioneller Arbeitsbogen zwischen Lehrkräften und Übungsleiterinnen und -leitern der Sportvereine in einem gemeinsamen pädagogischen System" (S. 16) entstehen. Im Folgenden soll dargelegt werden, wie sich dieser Interessenkonflikt konkret zusammensetzt.

Eine bundesweite Schulleitungsbefragung des IFS aus dem Jahr 2004 zeigte, dass in den etablierten Ganztagsschulelementen wie Arbeitsgemeinschaften, Dauer-Projekte oder Freizeitangebote in offener oder gebundener Form die Sportangebote „quantitativ deutlich an der Spitze“ (Holtappels, 2005, S. 21) liegen. Damit verschiedene Sportangebote zustande kommen können, müssen entsprechende Voraussetzungen vor Ort geschaffen werden. Persönliche Kontakte zu Trainern und Übungsleitern der jeweiligen Vereine spielen hierbei wie bereits erwähnt eine nicht unwesentliche Rolle, wenn es um die Kooperation zwischen Schu- 
le und Verein geht. Insbesondere im Wettkampfbereich des Sports und für die Talentsichtung und Talentsicherung ist eine gute Zusammenarbeit zwischen Lehrkräften und Vereinsverantwortlichen von großer Bedeutung. Die Entscheidung für das eine oder andere Angebot folgt dabei jedoch nur zur Hälfte einer „beschlossenen und konsenshaft abgestimmten pädagogischen Konzeption“ (Holtappels, 2005, S. 22), so die Studie des IFS. Weiterhin heißt es: „Diese Vorgehensweisen sind pragmatisch und verständlich, jedoch scheinen konzeptionellpädagogische Überlegungen eher zurückzustehen“ (ebd.). Folglich besteht laut dieser Aussagen Holtappels (2005) diesbezüglich noch Optimierungsbedarf.

Die Evaluationsstudien zum baden-württembergischen Landeskooperationsprogramm weisen darauf hin, dass sich nicht nur bei den Sportvereinen, sondern auch bei den Schulen die Attraktivität von Kooperationsgruppen „weniger auf erzieherische Zielsetzungen konzentriert, sondern eher auf eine erfolgreiche leistungssportliche Selbstdarstellung" (Fessler, 2003, S. 216). Hier ist mit Sicherheit ein Umdenken empfehlenswert. Die leistungssportlichen Wege zwischen Schule und Verein sollten überschritten und nach neuen Möglichkeiten für vielfältige Bewegungsaktivitäten gesucht werden, um eine Vielzahl von Kindern und Jugendlichen anzusprechen (Laging \& Hildebrandt-Stramann, 2006). Neben dem Leistungssport sollte demnach auch der Breiten- und Freizeitsport gefördert werden. Die Interessenslagen von Vereinen und Schulen sind demzufolge nicht immer identisch, im Gegenteil. Während die Schulen in ihrer Kooperation mit Vereinen in erster Line freizeit- und breitensportliche Angebote entwickeln und ihre SuS zum lebenslangen Sporttreiben motivieren möchten, haben die Sportvereine oftmals als oberstes Ziel, neue Mitglieder zu akquirieren sowie ihre Talentförderung anzukurbeln (LSB NRW, 2005). Im Gegensatz zur Schule ist der Sportverein außerdem traditionell an interessens- und altershomogenen Gruppen orientiert. Der Leistungs- und Konkurrenzgedanke ist stark ausgeprägt. An den Schulen hat Sport und Bewegung eher eine bildende, pädagogische und fördernde Bedeutung.

Fessler (2003) empfiehlt diesbezüglich eine „reflexive Koorientierung“ (S. 217) zwischen Schule und Verein. Nur so kann gewährleistet werden, dass beide Seiten und auch die SuS von passenden Bewegungsangeboten profitieren. Außerdem muss im Konzept von Ganztagsschulen vor allem der Partner Schule pädagogisch-konzeptionelle Überlegungen in den Vordergrund rücken. Darüber hinaus ist die Teilnahme an den von Vereinen angebotenen Sportarten anders als in den Schulen nicht verpflichtend, sondern freiwillig. Im breitensportlichen Bereich agiert der Verein mit vielen ehrenamtlichen Übungsleitern/innen und leider oft ohne professionelle Hauptamtlichkeit. In der Ganztagsschule werden diese Differenzen 
nun „zum Reibungspunkt und als Problem konstitutiv“ (Laging, 2006, S. 5). Er schlussfolgert aus diesem Dilemma:

„Die Schule möchte ein vielfältiges Bewegungs-, Spiel- und Sportangebot für alle oder möglichst vieler Kinder und auch die erreichen, die dem Sport nicht zugeneigt sind und der Verein muss sich im Gegensatz zu seiner auf Interesse und Freiwilligkeit beruhenden Orientierung auf die Heterogenität der Schülerschaft einlassen." (ebd.)

Laging (2006) bezieht sich in seinen Aussagen auf Naul (2006), der ein entstehendes Professionalisierungsproblem aufkommen sieht. Dadurch, dass viele Übungsleiter/innen ehrenamtlich arbeiten, besitzen diese folglich auch keine professionellen Kompetenzen für die schulischen Belange wie vergleichsweise studierte Lehrkräfte, was wiederum ein Professionalisierungsproblem verdeutlicht (Laging \& Hildebrandt-Stramann, 2006). Naul (2006) mutmaßt weiterhin, dass versucht wird, dieses Problem über Qualifizierungsmaßnahmen und Kooperationen mit den Landessportbunden zu beheben.

Dies sind ernstzunehmende Interessenskonflikte, denen man auch in Zukunft Beachtung schenken sollte. Die Schulen dürfen laut Laging (2006) hierbei ihre pädagogischkonzeptionellen Überlegungen keinesfalls aus den Augen verlieren und für „ein ausgewogenes Verhältnis von konzentriertem Lernen und Bewegen, von abwechslungsreicher Aktivität und Ruhe vor, zwischen und nach dem Unterricht oder auch in Verbindung mit dem Unterricht, von umfassender Entwicklungsförderung und Bildung in der bewegungsbezogenen Begegnung, von Förderung aller Kinder und Intervention bei sozialer Benachteiligung" (S. 4) sorgen. Zusammenfassend sollte in den Schulen „Bildung als aktive Selbstauseinandersetzung mit Bewegungsherausforderungen“ (ebd., S. 4) umgesetzt werden.

Neben all diesen Unterschieden weisen diese beiden Systeme aber auch Gemeinsamkeiten auf. Der Leistungsgedanke spielt sowohl in der Schule als auch im Verein die zentrale Rolle, auch wenn die Schulen dies oftmals verneinen. Die Notengebung im Fach Sport an den Schulen und die Ratifizierung und Beurteilung der SuS ist für jede Lehrkraft verpflichtend. Der Spaß am Spielen und Bewegen sowie all die nützlichen pädagogischen Chancen und Möglichkeiten der Persönlichkeitsbildung, welcher der Sport innehat, werden folglich vom Leistungsdenken verdrängt. Diese Schulkritik ist keineswegs neu und wurde schon bei mehreren KMKs ausgiebig diskutiert (Laging \& Hildebrandt-Stramann, 2006). 


\subsection{Das Basketball-Modell der Stadt Tübingen}

Neben den bereits erläuterten theoretischen Kooperationsmodellen zwischen Schulen und Vereinen soll im Folgenden ein real existierendes und praxisnahes Modell vorgestellt werden: Das Basketball-Modell der Stadt Tübingen.

Die Sportart Basketball wird in Tübingen bereits seit vielen Jahren auf der Ebene des Hochleistungssports betrieben. Die 1. Herrenmannschaft der sogenannten WALTER Tigers Tübingen - ehemals SV 03 Tübingen - spielten von 1903 bis 1982 sowie von 2002 bis 2017 in der 1. Basketballbundesliga.

Der Verein benötigt stets hochqualifizierte Spieler, aber woher soll er diese in einer Zeit des deutschen „Fußball-Booms" beziehen? Auch aufgrund der ungenügenden finanziellen Anreize, welche bei einer Randsport wie Basketball gegeben sind sowie durch die in Deutschland durchwegs mangelhafte mediale Unterstützung von Sportarten abseits des Fußballs, ist die Nachwuchsfindung sicherlich zusätzlich erschwert worden. Das führt dazu, dass Spieler nur durch gezielte Nachwuchsfördermaßnahmen sowie durch intensive Kooperationen zwischen dem Verein und weiterer Partner für den Basketballsport begeistert und mobilisiert werden können.

Damit dies möglich ist, sind selbstredend auch enge Kontakte zu örtlichen Schulen ein wichtiger Faktor. Fessler \& Ziroli (1997) sehen als vorrangiges Ziel des Basketball-Modells Tübingen „die Gewinnung möglichst vieler Kinder und Jugendlicher aus der Region Tübingen für den Basketballsport“ (S. 145) und bezeichnen als „wichtige Grundlage des Modells [ ] die enge Zusammenarbeit mit einer Vielzahl von Schulen in und um Tübingen“ (ebd.).

Da die schwäbische Stadt schon immer eine lange und erfolgreiche Tradition in dieser Sportart vorzuweisen hat, entschieden sich auch die umliegenden Gemeinden und Unternehmen bei diesem Projekt tatkräftig mitzuwirken. Neben den Schulen und Vereinen wurde ebenfalls ein Förderverein gegründet. Das Institut für Sport- und Sportwissenschaften der Stadt Tübingen versprach ebenfalls seine Unterstützung, wie auch der Basketballverband BadenWürttembergs. Somit konnte ein großes Netz an Kooperationspartnern gesponnen werden und die ca. 150 Jungen im Alter von 9 bis 18 Jahren erfuhren eine vorbildliche Förderung. Im Folgenden werden sämtliche Mitwirkenden dieses Basketball-Modells der Stadt Tübingen in einem Schaubild aufgezeigt: 


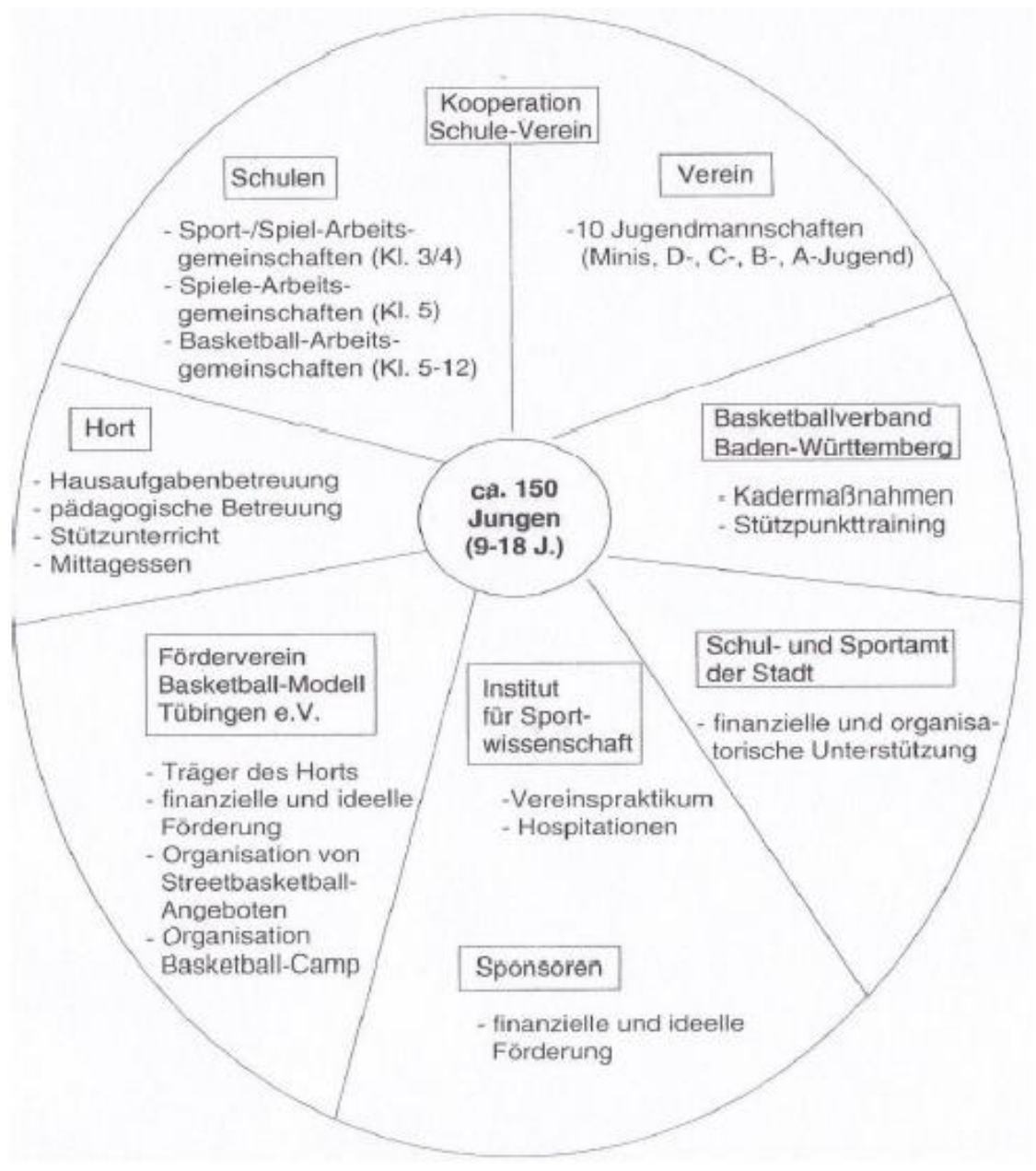

Abb. 7: Mitwirkende des Basketball-Modells Tübingen (Fessler \& Ziroli, 1997, S. 148)

Die einzelnen Aufgaben der mitwirkenden Kooperationspartner werden folglich erläutert:

- Der Schulsport soll die SuS in den Klassen 3 und 4 für den Basketballsport begeistern und in den weiterführenden Schulen (Klassenstufen 5 bis 12) sollen die Lehrkräfte im Sportunterricht insbesondere bei den Jungen einen Schwerpunkt in der Sportart Basketball setzen.

- Der Vereins- und Leistungssport bietet den ca. 150 Jungen im Alter von 9 bis 18 Jahren eine professionelle Betreuung und Ausbildung im Bereich Basketballteamsport an und ermöglicht den jungen Talentspielern die Teilnahme an Turnieren im Ausland, z.B. in Spanien oder Frankreich.

- Der Basketballverband Baden-Württembergs bietet den besonders begabten Spielern wöchentliches Sondertraining (Stützpunkttraining) an und bildet ebenso die Nachwuchsspieler zu Schieds- und Kampfrichter aus. 
- Im Schulhort wird den leistungssportorientierten SuS neben einer pädagogischen Nachmittagsbetreuung, Nachhilfeunterricht und ein Mittagessen angeboten.

- Die Stadt Tübingen unterstützt das Basketball-Modell finanziell und sorgt bei etwaigen Bundesligaspielen oder Turnieren für eine organisatorische Unterstützung.

- Das Institut für Sportwissenschaft integriert das Basketball-Modell in seine Veranstaltungspläne und bietet den Sportstudierenden der Uni Tübingen die Möglichkeit an, Vereinspraktiken und Hospitationen durchzuführen. Auch eine sportwissenschaftliche Betreuung des Bundesligateams wird vom Institut für Sportwissenschaften gewährleistet.

- Sponsoren werden immer wieder gesucht, um eine dauerhafte Absicherung des Projekts zu erzielen. Diese unterstützen den Verein in erster Linie finanziell und erhalten dafür Werbeflächen auf den Trikots, in den Spielzeitschriften oder der Halle. (Vgl. Fessler \& Ziroli, 1997, S. 144-148)

\section{Die acht Bausteine - Praktische Umsetzungsmöglichkeiten einer „Bewegten Schule“}

Laging (2006) nennt acht sogenannte Bausteine, welche explizite praktische Umsetzungsmöglichkeiten einer „Bewegten Schule“ beinhalten. Er nimmt damit Bezug auf die Zeitschrift Sportpädagogik (Heft 2/2001). Die Prioritätsgewichtung einzelner Bausteine kann sich von Schule zu Schule unterscheiden. Oftmals werden einige Bausteine von den Schulen gut entwickelt, währenddessen andere noch ausgebaut oder neu belebt werden müssen.

\subsection{Baustein 1: Bewegungsanlässe schaffen}

Durch gezieltes Platzieren von Geräten, Einrichtungen und Materialien auf dem Schulgelände kann das Sich-Bewegen-Wollen für SuS deutlich attraktiver gemacht werden. Räume sollen eine Art Appellcharakter bekommen und die Kinder und Jugendliche dazu auffordern, sich zu bewegen. Laging (2006) nennt folgende Umsetzungsmöglichkeiten:

- Körbe und Tore für Zielschuss- und Wurfspiele bzw. Ziele für Wurfgelegenheiten 
- Natürliche Geländeformen und -ausstattungen für Bewegungsaktivitäten zum Klettern, Rennen oder Versteckspielen

- Balancierbalken, Trecker- und LKW-Reifen, bewegliche Balken, Bretter und Holzkisten

- Rollgeräte für Asphaltflächen

- Sand- und Erdflächen als Gelegenheiten für Murmelspiele oder Spiele mit natürliche Materialien

- Bäume als Male oder Markierungen für Fang- und Nachlaufspiele (S. 7)

Zudem können Spielmaterialien und Sportgeräte in Schülerselbstverwaltung in den Unterrichtspausen bereitgestellt werden.

\subsection{Baustein 2: Schulhof als Bewegungs- und Ruheraum}

Eine klare Strukturierung und Ausweisung von Flächen, auf denen Spiel, Sport und Bewegung möglich sind, vereinfacht die Aktivitäten der SuS und kann zugleich motivationssteigernd wirken. Selbstverständlich können natürliche Gegebenheiten wie Hecken, Bäume und Büsche, aber auch die Bodenflächen wie Sand, Pflasterstein, Erde, Rasen oder Mulche dazu dienen, gewisse Funktionsflächen voneinander abzugrenzen oder zu kennzeichnen. Bei einer Neu- oder Umgestaltung solcher Flächen ist es unbedingt von Nöten, Expertenwissen miteinzubeziehen. Die Gestaltung eines solchen Bewegungsraums kann so perfektioniert und die Motivation der SuS durch eine optimale Raumstruktur gefördert werden (Laging, 2006).

\subsection{Baustein 3: Bewegungsraum Schulgebäude}

Nicht nur im Freien kann eine bewegte Schulkultur gelebt werden, sondern auch im Schulgebäude, das laut Laging (2006) als Bewegungsraum genutzt werden sollte. So könnten diverse Klassenzimmer zu Bewegungsräumen umgebaut werden. Nach dem Prinzip der Bewegungsbaustelle (Miedzinski, 2000) können diese Räume mit unterschiedlichen bewegungseinladenden Gegenständen wie Brettern, Strickleitern und Würfeln ausgestattet oder feststehende Installationen wie Klettertürme oder Rutschen eingebaut werden. Natürlich darf der eigentliche Unterricht im Verhältnis zu den Bewegungsaktivitäten nicht ins Hintertreffen geraten. Viele SuS könnten durch diese Bewegungsangebote jedoch zielgerichteter lernen, auch wenn die eben genannten Gegenstände innerhalb des Unterrichts nur eingeschränkt 
genutzt werden würden. Lediglich die vorhandene Möglichkeit der Bewegung beruhigt unkonzentrierte SuS, da diese wissen, im Notfall ihren Bewegungsdrang ausleben zu können. Es müssen darüber hinaus nicht ausschließlich kostenintensive und offensichtliche Bewegungsangebote in den Klassenzimmern angebracht werden. Stehtische oder anderweitige Lernorte, welche die SuS zum Stehen oder Liegen animieren, durchbrechen das weitverbreitete und ungemein gefährliche sedentäre Verhalten der Kinder und Jugendlichen und würden Bewegung in ihren Lernalltag bringen.

Tischtennisplatten, Fitnessgeräte, Tischkicker oder Billardtische können zudem ebenfalls im Inneren eines Schulgebäudes platziert werden - Laging (2006) spricht die Empfehlung aus, dass die SuS diese jedoch ausschließlich nach vereinbarten Regeln nutzen dürfen. Neben diesen eher funktionsorientierten Räumen sollte die Gestaltung der Flure ebenfalls berücksichtigt werden. Helle, freundliche und weite Räume laden mehr zur Bewegung und Interaktion ein als dunkle, enge Räume. Durch einen verstärkten Einsatz von Pflanzen und Sitzmöglichkeiten wäre es zudem möglich, den SuS neben den bewegungsorientierten Räumen, Orte der Ruhe zu bieten.

\subsection{Baustein 4: Bewegtes Lernen und Unterrichten}

Wie bereits erwähnt, muss kognitives Lernen nicht ausschließlich im Sitzen durchgeführt werden. Der Bewegungsdrang sowie die Freude und Lust der Kinder und Jugendlichen am Spielen sollten laut Laging (2006) nicht dermaßen unterbunden werden, wie es im reinen Sitzunterricht an den meisten deutschen Schulen der Fall ist. Der Bezug zur Lebenswelt der Kinder muss von den Lehrkräften respektiert und berücksichtigt werden - so können mit hoher Wahrscheinlichkeit ungeahnte Ressourcen in der Lehr- und Lerngestaltung sowie im Lernerfolg der SuS freigesetzt werden. Laging (2006) nennt bspw. unterschiedliche „Lernecken, Materialregale, einen Leseteppich, Spielnischen, Regale mit Lernhilfen“ (S. 9), welche in die Klassenräume integriert werden könnten. So entstünde aus dem Klassenzimmer eine Art Lernlandschaft - der Unterricht könnte so immens belebt werden und durch das Stationenlernen wären die SuS auf Bewegung angewiesen. Darüber hinaus bieten sich eine derartige Gestaltung der Klassenräume sowie die bewegungsorientierte Unterrichtsgestaltung anhand der Themen vieler Schulfächer ohnehin an, da SuS gewisse Lerninhalte „durch ein bewegtes Begreifen besser leiblich durchdringen und das Wissen auch zu einem Körperwissen transformieren" können (Laging, 2006; vgl. Hildebrandt-Stramann, 1999). Zudem nennt Laging (2006) die Möglichkeit innerhalb des Unterrichts eine oder mehrere Bewegungspau- 
sen durchzuführen, damit die SuS durch gezielte Bewegungsangebote ihre Konzentrationsfähigkeit wiederherstellen können.

\subsection{Baustein 5: Bewegtes Sitzen}

Wie können Kinder und Jugendliche am besten Lernen? Ein Blick in den Schulalltag an deutschen Schulen zeigt, dass der „Standardunterricht“ im Stillsitzen auf herkömmlichen Stühlen stattfindet. Zum einen wird dadurch das sedentäre Verhalten der Kinder und Jugendlichen durch das stundenlange Sitzen zusätzlich gefördert. Zum anderen wird hierbei ebenso nicht berücksichtigt, dass manche SuS besser im Liegen lesen oder im Stehen lernen können. Ein gleichmäßiger Wechsel der Halte- und Sitzpositionen würde das sedentäre Verhalten durchbrechen sowie die unterschiedlichen Lerngewohnheiten der Kinder und Jugendlichen besser einkalkulieren. Laging (2006) empfiehlt zusätzlich, ein flexibles Sitzmobiliar und unterschiedliche Sitzgelegenheiten in die Klassenräume zu integrieren wie bspw. Bälle, Quader oder Stehsitze. Sobczyk und Landau (2003) sowie Breithecker (1996) haben bereits bewiesen, dass durch ein aktivdynamisches Sitzen und Belastungswechsel eine natürlichere Beanspruchung des Halteapparats und eine Belebung des Lernverhaltens von SuS erreicht werden kann.

\subsection{Baustein 6: Bewegter Schultag}

Unterrichtsstunden sind an deutschen Schulen in 45 minütige Einheiten gegliedert. Ein rhythmischer Wechsel von Ruhe und Bewegung wird hierbei nicht berücksichtigt. Laging (2006) ist jedoch der Meinung, dass sich ein bewegter Schulalltag nur dann realisieren lasse, wenn dieser Ablauf rhythmisiert werde. Um dieses Konzept zu ermöglichen, wäre es sinnvoll die sogenannte Gleitzeit in die Schulen zu integrieren. Dieses Gleitzeitkonzept haben auch schon einige deutsche Schulen umgesetzt (vgl. Röhner, Skischus \& Thies, 1998). In dieser Gleitzeit können die SuS bspw. Aufgaben bearbeiten, mit anderen Kindern und Jugendlichen spielen, sich bewegen, unterhalten oder Ruhe suchen. Darauffolgend kann in einem ersten Unterrichtsblock mit den SuS konzentriertes und bewegtes Arbeiten im Wechsel, Frontalunterricht oder Gruppenarbeiten angewendet werden. Im Anschluss daran wird den SuS eine Frühstückspause sowie eine ausgiebige Bewegungs- und Spielpause ermöglicht. Dieser Block dauert ebenfalls 45 Minuten an. Daraufhin folgt ein weiterer Unterrichtsblock der laut Laging (2006) stärker projektbezogen sein sollte, d.h. es wird eine Unterrichtsform gewählt, die von ihrem Charakter bewegungsorientiert ist. Nach einer 60 minütigen Mittagspause hätten SuS 
von gebundenen Ganztagsschulen darüber hinaus noch Lernzeit, die mit Bewegungsangeboten, AGs oder Fachunterricht gefüllt werden könnte. Offene Ganztagsschulen können selbstverständlich ebenfalls Sportangebote oder Betreuungszeiten anbieten.

\subsection{Baustein 7: Bewegungsangebote, Spielfeste und Öffnung von Schule}

Wie bereits mehrfach erwähnt, ist durch die Verlängerung des Schultags eine Ganztagsschule aus rein zeitlichen Gründen spielend leicht dazu in der Lage, nach der Mittagspause viele unterschiedliche und außerschulische Bewegungsangebote anzubieten. Diese müssen sich nicht ausschließlich an den normierten und traditionellen Sportarten wie Fußball, Basketball oder Leichtathletik orientieren. Bogenschießen, Ringen und Raufen, Parcours sowie Zirkusund Akrobatikkünste wären hierfür Beispiele. Außerdem sollten vermehrt Bewegungs- und Sportfeste als rein schulische, aber auch als schulübergreifende Veranstaltungen integriert werden. Wettkämpfe, in denen die SuS dazu in der Lage sind, sich in einen sportlichen Wettstreit zu begeben haben hierbei genauso ihre Berechtigung wie sogenannte Spielfeste, in denen das Miteinander und nicht das Gegeneinander bewegen im Vordergrund steht.

\subsection{Baustein 8: Bewegter Sportunterricht}

Dem Sportunterricht muss in einer bewegungsorientierten Schule folglich ein hoher Stellenwert zugesprochen werden. Sport als Unterrichtsfach stand in den vergangenen Jahren mehrfach in einer Legitimationskrise. Die bereits genannten medizinischen Studien sowie Lagings (2006) Auffassung, dass der Sport ein intentionales und sinnbesetztes Bewegungshandeln bei Kinder und Jugendlichen initiiert, welches für die Persönlichkeitsentwicklung ungemein wichtig ist, sollte dieser Legitimationskrise entgegen wirken. Im Sportunterricht sollen nicht nur die normierten Sportarten mit ihren festen Techniken und Regeln gelehrt werden. Ein moderner Sportunterricht beschäftigt sich vielmehr mit bewegungsthematischen Situationen und Bewegungsabsichten.

Laging (2006) nennt diesbezüglich das Bewegungsfeld Turnen als Beispiel. Beim Turnen kann die Bewegungsabsicht das Springen (über einen Sprungtisch) und Stützen (auf einem Barren) sein. Die SuS können Bewegungserfahrungen sammeln und eigene Lösungen finden. Diese Erfahrungen können von den Lehrkräften beliebig variiert werden. Die Selbstaktivierung der SuS sollte jedoch stets unterstützt werden, denn durch eigeninitiiertes und selbsttätiges Handeln können Inhalte und in diesem Fall spezifische Bewegungen am besten verinnerlicht 
und gelernt werden. Attraktive Bewegungsangebote können die intrinsische Motivation von SuS enorm steigern. Dies kann ebenfalls durch sogenannte Wunschsportstunden, in denen die Kinder und Jugendlichen selbst die Initiative übernehmen (vgl. Funke, Heine \& Schmerbitz, 1979; Laging, 1993) gefördert werden. Laging (2006) bringt es folgendermaßen auf den Punkt: „Ein erfahrungsorientiertes Lernen ist einer durchgreifenden Belehrung vorzuziehen“ (S. 11).

\subsection{Fazit und Diskussion}

Diese Bausteine können im Optimalfall eine Verbesserung des bewegungsaktiven Lebensstils Kinder und Jugendlicher mit sich bringen. So kann den empirischen Studien zum Bewegungsumfang von Kindern, welche höhere Bewegungszeiten fordern, entsprochen werden. Wichtig ist, dass ausreichend herausfordernde Räume für Bewegungsaktivitäten eröffnet werden - sowie in informellen als auch in nonformalen Settings im Kindes- und Jugendalter. Damit ist gemeint, dass nicht nur explizit die Schulräume, sondern auch die Wege zwischen Schule und Zuhause oder das Wohnumfeld selbst bewegungsfördernd gestaltet werden müssen.

Die Ganztagsschulen könnten durch integrative Konzepte einen wertvollen Beitrag dazu leisten, dass Bewegung zum Alltag der Kinder und Jugendlichen wird - und das eben nicht nur im Unterricht, sondern auch in der Freizeitgestaltung vieler SuS. Laging (2006) empfiehlt, dass diesbezüglich die selbstorganisierten informellen Bewegungsmöglichkeiten deutlich mehr gestärkt werden müssen. Zudem müssen die SuS lernen, „sich Räume aktiv bewegungsorientiert zu erschließen“ (Laging, 2006, S. 11), denn Schule ist nicht nur ein Lern- und Lebensraum, sondern vor allem ein Bewegungsraum.

\section{Bedeutung von Bewegung und Sport in informellen und nonformalen Settings im Kin- des- und Jugendalter}

Die folgenden Seiten analysieren, inwieweit informelle und nonformale Bewegungsräume als Handlungs- und Lernorte von Kindern und Jugendlichen dienen und betrachten in diesem Zusammenhang die Bedeutung des Sports in solch einem Handlungsumfeld. Dabei werden folgende Aspekte behandelt: Wie und was lernen Kinder und Jugendliche durch informelle und nonformale sozialräumliche Bildung? Welche Bedeutung kommt der körperlichen Aktivität beim Bildungsprozess im Raum zu? Unter welchen Voraussetzungen lernen Kinder im Raum am besten? Und schließlich, wann und inwiefern ist das Lernen in nonformalen und 
informellen Settings heutzutage eingeschränkt? Letztlich werden im Ausblick stadtplanerische Perspektiven genannt, die informelle und nonformale Lernumgebungen im öffentlichen Raum unterstützen.

Es werden drei sozialräumliche Bildungsformen unterschieden: formelle, nonformale und informelle Bildungsprozesse. Formelle Bildungsprozesse beziehen sich auf Bildung durch das staatliche Bildungssystem, also die schulische Bildung. Auf diese Bildungsform wird im Folgenden nicht weiter eingegangen, da sie innerhalb dieser Abschlussarbeit bereits ausgiebig diskutiert wurde. Vielmehr werden die non-formale und informelle Bildung in den weiteren Mittelpunkt der Untersuchungen gerückt. Anders als in formellen Bildungsprozessen, in denen das Lernen von einer Lehrkraft angeleitet wird, beinhaltet informelle Bildung ungeplante und nicht-intendierte Bildungsprozesse, die sich im Alltag von Familie, Nachbarschaft, Arbeit und Freizeit ergeben. Nicht-formelle bzw. nonformale Bildung steht als Bildungsform zwischen den beiden genannten Formen. Hier handelt es sich zwar um organisierte Bildung und Erziehung, diese ist jedoch generell freiwilliger Natur wie bspw. Angebote durch Jugendoder Vereinsarbeit (Deinet, 2010).

\subsection{Raum und Raumaneignung}

Informelle Bildungsprozesse können grundsätzlich überall stattfinden. Als mögliche Lernorte informeller Bildung im Hinblick auf Bewegung und Sport kommen insbesondere öffentliche Räume in Betracht, bei denen genügend Platz für körperliche Aktivitäten zur Verfügung steht. Eine klassische informelle Bewegungsaktivität von Kindern ist z.B. das „Toben und Spielen“ im Freien. Das kann auf der Straße, aber auch im Park oder Wald stattfinden. Des Weiteren kann auch der Schulweg ein Ort informeller Bildung sein (Brandl-Bredenbeck \& Brettschneider, 2010). Nissen differenziert indessen drei verschiedene Typen von öffentlichen Räumen, die als Orte informeller Bildung dienen können: öffentliche Freiräume wie Parks, Spielplätze oder Straßenraum, öffentlich zugängliche, verhäuslichte Räume wie Kaufhäuser oder U-Bahnhöfe sowie institutionalisierte, öffentliche Räume wie Sportanlagen, Schulräume oder Vereinsheime (Deinet, 2004).

Nun steht diesem absoluten Raumverständnis, welches den Raum als eigentliche Realität begreift (Bsp.: Ein Spielplatz ist eine Fläche auf dem Objekte zum Spielen stehen), ein relatives Raumverständnis gegenüber, das sich auf den unmittelbaren Zusammenhang zwischen der Aktivität des Menschen und der Konstitution von Raum bezieht. Der Mensch ist hier beim Raumentwurf inbegriffen und der Raum konstituiert sich aus der Folge von Bewe- 
gungshandlungen (Löw, 2009). Laut Foucault ist das Zusammenspiel von Mensch und Raum ein reziprokes Verhältnis, in welchem der Raum im Handeln strukturiert wird oder Handeln strukturiert (Löw, 2001). Zudem sieht Foucault Raum als ein dynamisches Gebilde: Die Beschaffenheit von Räumen ist nie starr, sondern prozesshaft. Ein Raum besteht demnach aus mehr als Erdboden oder Wänden. Vielmehr entstehen und wandeln sich Räume durch die Erlebnisse, die dort stattfinden (ebd.).

Die Beschaffenheit von Raum entsteht somit durch zwei Prozesse: Zum einen durch das sogenannte "spacing“, welches das Schaffen oder das Kreieren von Raum darstellt, also dem Positionieren in Relation zu anderen Platzierungen. Zum anderen wird Raum durch eine sogenannte „Syntheseleistung“ über Vorstellungs-, Wahrnehmungs- oder Erinnerungsprozesse konstituiert (Löw, 2001). Dabei können auch durch die Aktivität verschiedener Gruppen mehrere Räume an einem Ort entstehen. So kann eine öffentliche Tischtennisplatte ein ambivalentes Objekt darstellen. Für die einen ist sie ein Sportgerät zum Tischtennisspielen, für andere wiederrum dient sie als Treffpunkt oder als Liegemöglichkeit zum Entspannen (ebd.). Die Konstitution von Räumen geht einher mit der Aneignung von Räumen. Holzkamp (1983) lehnt sich mit seinem Aneignungskonzept an die materialistische Aneignungstheorie von Leontjew (1973) an. Darin sieht er die Aneignung als schöpferischen Prozess der eigenen Auseinandersetzung mit der Gestaltung und Veränderung von Räumen und Situationen (Deinet, 2010). Durch die aktive Auseinandersetzung mit der Umwelt fördert Raumaneignung die Entwicklung des Individuums und ist somit eine Selbst- oder Subjektbildung. Dadurch ist Raumaneignung an sich schon ein informeller Bildungsprozess (Deinet, 2010). Kinder und Jugendliche erlernen durch die Aneignung öffentlicher Räume Eigenständigkeit und entwickeln Schlüsselkompetenzen wie Handlungskompetenz, Risikoabschätzung, Neugier und Offenheit. Zudem fördert Raumaneignung die Erweiterung motorischer und kreativer Kompetenzen (Deinet, 2004). Raumaneignung kann folglich mentale, aber auch motorische Bildungsprozesse beinhalten.

Jacobs (1992) nennt hinsichtlich der Aneignung von Raum noch weitere Lernprozesse, die im öffentlichen Raum zwischen Menschen stattfinden können: Toleranz im Umgang mit fremden Lebenssituationen, die reflektierte Verarbeitung von Fremdheit und Unterschiedlichkeit, Rücksichtnahme auf Schwächere und Verantwortungsbewusstsein. 


\subsection{Bedeutung von Sport für Raumaneignung}

Das Vordringen in Räume findet nicht nur dadurch statt, indem sich Individuen in einer Raumrelation positionieren. Gleichermaßen kann durch Sport Raum angeeignet werden. Doch Raumaneignung und Sport bedingen sich nicht nur gegenseitig über den Aspekt der Bewegungshandlung. Sie haben zudem beide eine immense Bildungsfunktion, wodurch sie im Prozess der Selbstsozialisation eine wichtige Rolle spielen (Scherr, 2004; BrandlBredenbeck \& Brettschneider, 2010). Sobiech (2002) bezeichnet die Aneignung von Räumen als unabdingbar für die Bildung des sogenannten „sozialen Kapitals“, das zum ersten Mal von Bordieu erwähnt wurde. Dieses soziale Kapital stellt angeblich die entscheidende Voraussetzung für sozialen Erfolg, Durchsetzungsfähigkeit und Verwirklichung der eigenen Ansprüche und Interessen dar.

Durch Sport und Spiel können Kinder und Jugendliche im Optimalfall soziale Fähigkeiten erlernen und ein geschlechts- und klassenspezifischer Umgang mit dem Raum trainiert werden. Diese Fähigkeiten sind auch für viele späteren Lebenssituationen von wesentlicher Bedeutung (Sobiech, 2002). Laut Burmann (2008) lernen Kinder und Jugendliche darüber hinaus durch Sport im informellen Rahmen Verantwortung und Selbstständigkeit, denn informelle Bewegungs-, Spiel- und Sportaktivitäten werden durch die Beteiligten situationsgebunden häufig selbst arrangiert, geplant und durchgeführt. Burrmann (2008) deutet auf die Wichtigkeit hin, dass die zeitlichen, räumlichen und sozialen Rahmungen durch die Teilnehmer/innen selbst vereinbart und definiert werden. Ferner ist er der Meinung, dass Kreativität, Sozialität, Subjektivität und Stilisierung in informellen Sport- und Bewegungsaktivitäten gegenüber dem vereinsorientierten Sport sehr viel stärker in Erscheinung treten.

Scherr (2004) hingegen weist auf die im Sport geschulte Kommunikationsfähigkeit hin und verdeutlicht, dass unterschiedliche Sozialräume Erfahrungs-, Begegnungs- und Kommunikationsmöglichkeiten eröffnen, aber auch verschließen könnten. Im Sport kommt es laut Deinet (2004) für die Kommunikations- und Beziehungsnetzwerke daher auch auf den Raum an, da dort in körperlicher Zusammenarbeit die räumliche Kopräsenz konstitutiv ist.

So stellt sich die Frage, wie ein Raum aussehen sollte, in dem Kinder und Jugendliche am besten lernen. Burrmann (2008) sieht eine (Wohn-)Umwelt als spiel- und entwicklungsfördernd an, welche das Kind dazu anregt, aktiv und spontan zu handeln und es ihm dadurch ermöglicht, in unbekannte Bereiche vorzudringen. Öffentliche Räume als informelle Umgebung sind demnach Bereiche, in denen viele Lernprozesse stattfinden können. Als Ort der

Öffentlichkeit sehen Podlich und Kleine (2003) den Anreiz der Straße, denn dort können sich 
Kinder besonders frei inszenieren und präsentieren. Weiterhin bietet die Straße einen Ort der Offenheit, der große Freiheit für Bewegung und Kreativität ermöglicht. Als Ort der Bewegung erhält sie eine zusätzlich gesundheitsfördernde Bedeutung, weshalb Podlich und Kleine (2003) fordern, die Alltagsmobilität als ernstzunehmenden Bewegungs- und Entwicklungskatalysator von Kindern anzuerkennen. Sie plädieren daher u.a. dafür, Bewegungen der Alltagsmobilität, wie das Radfahren, zu fördern und dem Busfahren vorzuziehen.

\subsection{Einschränkungen in der Entwicklung des Städtebaus}

In den letzten Jahrzehnten hat die Entwicklung des Städtebaus die Bedeutung der Straße und anderer informellen Lernsettings für Kinder und Jugendliche in den Hintergrund treten lassen. Deinet und Reutlinger (2004) bezeichnen die heutige Stadt als unternehmerische Stadt. Bewegungsflächen stehen immer häufiger in Konkurrenz zu Bauvorhaben, die den Fokus auf den Bau großer Konzerne legt und nur in seltenen Fällen den (Bewegungs)Bedürfnissen der Zivilbevölkerung nachgeht (Deinet, 2004). Die Folge ist eine erkennbare Verringerung von Angebotsdichte und Qualität von Bewegungsräumen, die in einer Anregungsarmut resultiert.

Oft fehlen laut Breckner (2010) institutionelle Strukturen, die Bewegung im öffentlichen Raum in sozial und kulturell angemessener Weise anregen. Zusätzlich ist immer häufiger erkennbar, dass viele öffentliche und private Bewegungsräume in Schulen, Schwimmbädern, Sportvereinen, Betrieben und Universitäten einschränkenden Nutzungsbedingungen unterliegen, die in vielen Fällen eine Unternutzung oder monofunktionale Nutzung erzeugen (Breckner, 2010). Um diese Beschränkungen durch Nutzungsrestriktionen und Monofunktionalität zu verdeutlichen, nennt Breckner (2010) bspw., dass heute zunehmend Zaunbauten und Erdwalle um Bewegungsflächen gebaut würden, um mögliches Spielen und Bewegen auf diesen Flächen und einen allgemeinen Zugang für bestimmte Nutzergruppen zu verhindern. Deinet und Reutlinger (2004) sind außerdem der Auffassung, dass der Bauboom im Zuge der Modernisierung in Städten stetig problematischer wird, da durch den Verlust von disfunktionalen Flächen soziale Probleme entstünden: Kinder werden dadurch mehr und mehr „verhäuslicht" und institutionalisiert, da sie nur noch in der Schule oder im Verein einer Sportaktivität nachgehen können. Burrmann (2008) schließt sich dieser Meinung an und bestätigt, dass sich die Lebenswelt der Kinder radikal verändert hat und spricht vom Wandel der "Straßenkindheit" zur „verhäuslichten“ und „verinselten“ Kindheit. 


\subsection{Sport und Verinselung}

Im Folgenden soll das Phänomen der „Verinselung“ erläutert werden. Wenn Kinder ihren Sozialraum erkunden und entdecken, geschieht dies häufig vom familiären Zuhause aus. Die Ausweitung des Sozialraums, so schreibt Baacke (1980), kann vor allem für frühere Generationen in Form von konzentrischen Kreisen beschrieben werden, welche er als Zonenmodell charakterisiert. Die vier Zonen, die sich vom Zentrum über den Nahraum bis in die Peripherie erstrecken, bieten den Heranwachsenden dabei verschiedene Erfahrungs- und Erlebnismöglichkeiten und stellen unterschiedliche Anforderungen an sie.

Die Erweiterung des Sozialraums von Kindern und Jugendlichen der heutigen Generation nähert sich jedoch eher dem Inselmodell von Zeiher (1983) an. Dieses sieht wiederum den Lebensraum nicht als ein Segment an, sondern als viele separate Stücke, die wie Inseln verstreut in einem größeren Gesamtraum liegen:

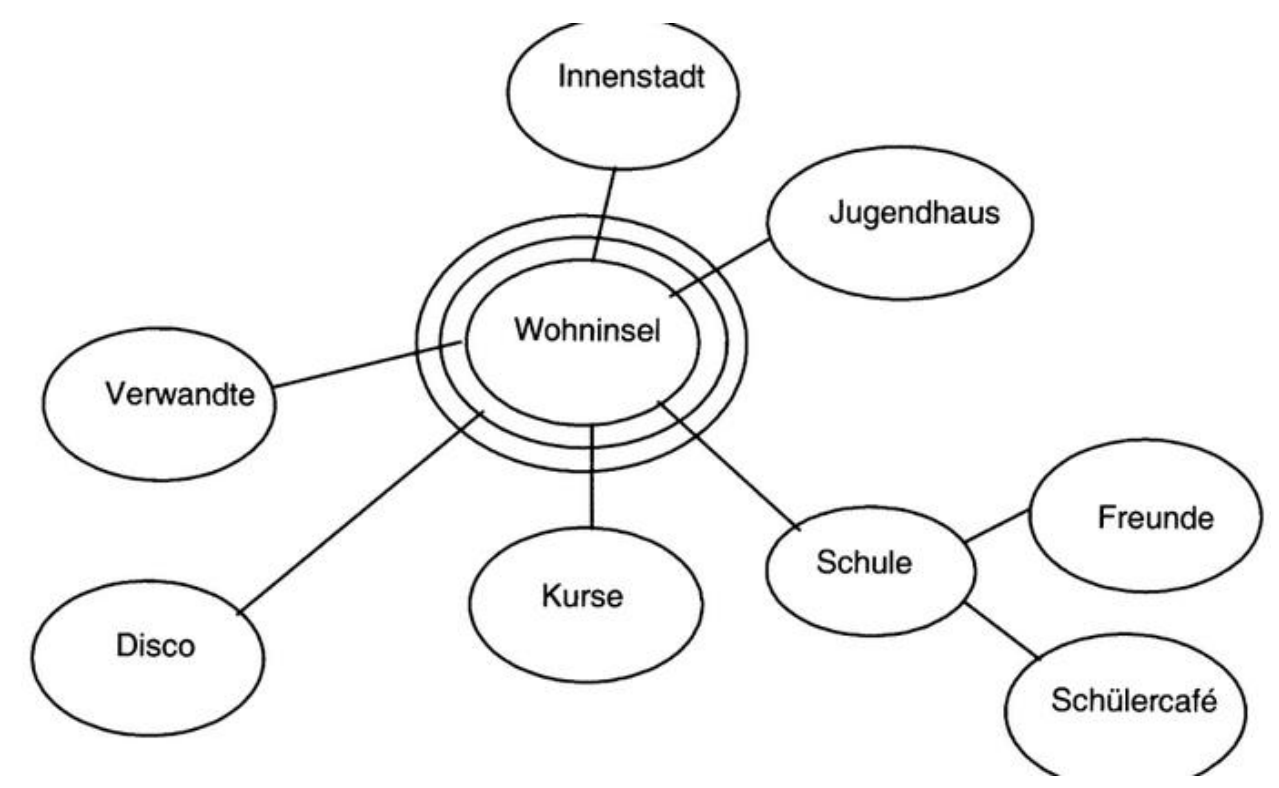

Abb. 8: Inselmodell nach Zeiher (Quelle: Deinet, 2005, S. 48)

Außerhalb dieser Inseln ist der Gesamtraum an sich bedeutungslos, da dieser Raum zwischen den Inseln durch Auto- oder Bahnfahrt überbrückt und daher nicht wahrgenommen wird. Kritiker des heutigen städtebaulichen Wandels bemängeln, dass durch die zunehmende Verinselung eine ganzheitliche Wahrnehmung des Gesamtraums beeinträchtigt wird und es dadurch zu einer Zerstückelung des Raums kommt, bei dem das homogene Gesamtkonstrukt aufgelöst wird (Löw, 2001). Die Auflösung homogener Raumvorstellung durch die Verinselung führt schließlich zur Desozialisation der Kinder und Jugendlichen, was einen Verlust von Kontinuitätserleben und Konsistenzgefühlen zur Folge hat (Löw, 2001). 
In Bezug auf Sportaktivitäten wird ferner angenommen, dass die wachsende Verinselung sich nachteilig auf das Bewegungsverhalten von Kindern auswirkt, denn wohnortnahe Sport- und Spielgelegenheiten sind laut Burrmann (2008) immer seltener erreichbar.

Die fortschreitende räumliche Differenzierung zeigt sich vor allem in Großstädten, in denen Bewegungs- und Sportaktivitäten nur noch an bestimmten Orten wahrgenommen werden können (ebd.). Die (sportartspezifischen) Handlungs- und Bewegungsräume sind ergo zunehmend festgelegt. Eine freie Entfaltung und Erschließung von Räumen Kinder und Jugendlicher wird somit gehemmt und eine weitere Verinselung dadurch gefördert. Diese Spezialisierung und Differenzierung von Bewegungsraum hat zur Folge, dass Kinder heutzutage vor allem organisierte Sportangebote wahrnehmen, da die von Kindern früher weitgehend eigenverantwortlich organisierten Freizeitaktivitäten zunehmend an institutionelle Orte verlagert werden (ebd.). Daher treten informelle Bewegungsaktivitäten zunehmend in den Hintergrund. Diese steigende Institutionalisierung von Kindern ist zusätzlich dafür verantwortlich, dass die Kindheit immer mehr von festen Terminen und Verbindlichkeiten geprägt wird (Sobiech, 2002). Nicht selten wird dieses Phänomen von den Eltern selbst geschaffen, weil sie als erste Instanz ihrer Kinder den Sportverein als Einrichtung und Lösung der Bewegungseinschränkung wählen. Auch ein mögliches Ganztagsschulkonzept würde dieser umstrittenen Entwicklung folgen. Wenn die Kinder und Jugendlichen bis zum späten Nachmittag in der Schule sein müssen, ist es nahezu unmöglich, dass sie selbst organisierten, informellen Bewegungsformen nachgehen können.

Die Inbesitznahme von Bewegungsräumen erfolgt somit vielfach in Begleitung und damit unter sozialer Kontrolle Erwachsener (Burrmann, 2008). Aufgrund von zunehmender Reglementierung durch Erwachsene, zunehmenden Verkehr, den wenigen Spielanregungen für Kinder und den vielen weiteren genannten Faktoren beschreibt Dietrich (2001) die heutige Großstadt als Spielwüste (2001). Für Kinder und Jugendliche sind Stadträume heute in ihrer Funktion eindeutig festgelegt und daher nicht mehr deutbar. Dies erschwert ein kollektives Zusammenspielen und viele damit einhergehende Lernprozesse deutlich.

Die Wohnlage ist auch ein wichtiger Faktor, der für mögliche Nutzungsbeschränkungen des öffentlichen Lernraums sorgen kann. Ungünstige Wohnumweltbedingungen, die sich durch weniger interessante Spiel- und Freizeitmöglichkeiten, weniger Natur, Angst vor Kriminalität, viel Verkehr und ungünstige Bus- uns Bahnverbindungen kennzeichnen, wirken sich negativ auf das Bewegungsverhalten von Kindern aus, während Kinder, die in günstigen Wohnumweltbedingungen leben, sich häufiger draußen bewegen um bspw. Rad oder Skateboard zu 
fahren oder im Garten zu spielen (Schmidt, 2008). Des Weiteren zeigt sich, dass ungünstige Wohnumweltbedingungen häufig mit dem sozial niedrigeren Status und einem Migrationshintergrund korrelieren, was die Benachteiligung sozialschwacher Kinder weiter verstärkt. Das Heranwachsen Kinder und Jugendlicher dieser Gruppen hinsichtlich der Förderung eines gesunden Lebensstils wird folglich ebenfalls erschwert (Brandl-Bredenbeck \& Brettschneider, 2010).

\subsection{Unterschiede in der Raumaneignung von Jungen und Mädchen}

Allerdings sind die eben genannten Faktoren nicht die einzigen Gründe für eingeschränkte Sport- und Bewegungsaktivitäten in informellen Settings. Denn selbst wenn Wohnbedingungen und sozioökonomischer Status ausgewogen sind, ist zu erkennen, dass Unterschiede zwischen Mädchen und Jungen in der Aneignung von Sport-Spiel-Räumen, aufgrund von geschlechtertypischen Sozialisationsprozessen, bestehen (Burrmann, 2008). Die Aneignung und Nutzung von Räumen in Sport und Spiel bedingen sich gegenseitig. Außerdem sind sie äußerst eng mit der Fähigkeit Kinder und Jugendlicher verwoben, in spielerischer oder sportlicher Betätigung sich diese Räume anzueignen (Sobiech, 2002).

Flade und Kustor-Hüttl (1993) argumentieren, dass fälschlicherweise die Auffassung „Kinder spielen überall“ (zitiert nach Dietrich, 2001) in erster Linie für Jungen gelte. In ihrer Studie belegten sie, dass auf öffentlichen Plätzen nur knapp 20 \% der spielenden 10-Jährigen, Mädchen waren (ebd.). Diese Forschungsergebnisse sprechen dafür, dass Jungen sich bei der Aneignung von Räumen gegenüber Mädchen häufiger durchsetzen. Ein Erklärungsversuch hierfür könnte u.a. sein, dass sich Jungen in Sportspielen Mädchen gegenüber in Leistungsund Konkurrenzprinzipien, wie dem Überbieten des gegnerischen Teams oder der Einteilung in Gewinner und Verlierer, behaupten (Sobiech, 2002). Da Jungen häufiger als Mädchen dazu tendieren in Konkurrenz mit anderen zu treten, sich messen und (Körper-)Grenzen austesten wollen, wäre dies eine weitere Erklärung dafür, dass Jungen in der Aneignung von Räumen den Mädchen überlegen sind (ebd.).

Als weitere Phänomene sind das Austragen von Konflikten, Aushandeln von Spielregeln und Abstimmen taktischer Maßnahmen Bestandteile der sozialen Entwicklung, die als Lernerfahrungen innerhalb des Sportspiels entstehen können. Sobiech (2002) sieht durch die erlernten Prinzipien einen profitablen Nutzen für die Alltagswelt, auf die sie sich übertragen lassen, da sie insbesondere auf dem Arbeitsmarkt und in der wirtschaftlichen Konkurrenz von Bedeutung sind. Das erwerben von Überlegenheit, Durchsetzungsvermögen und Erfolg sind wichti- 
ge Charakteristika, um auf modernen Arbeitsmärkten und im wirtschaftlichen Wettbewerb mithalten zu können (ebd.). Weiter argumentiert er, dass all diejenigen, die nicht über ein solches soziales Kapital verfügen, sich selbst ausschließen oder aus dem Spiel ausgeschlossen werden. Diesen Auffassungen stehe ich eher kritisch gegenüber. Nicht in jeglichen Berufen sind Charaktereigenschaften wie Überlegenheit und der unbedingte Wille zum Erfolg dafür ausschlaggebend, ob ein Mensch seine Aufgaben im beruflichen Leben gut oder schlecht bewältigen kann. Auch die zuletzt genannte These, dass all diejenigen, die nicht über ein solches soziales Kapital verfügen, sich selbst ausschließen, halte ich für überzogen und realitätsfern. Insbesondere in sozialen Berufen greift dieses Argumentation in keiner Weise.

Im Hinblick auf Raumaneignung scheinen Mädchen anhand der eben dargelegten Beobachtungen eher zurückhaltend zu sein, insbesondere wenn dieser Raum mit Nutzung anderer männlicher Gruppen konkurriert. So tendieren weibliche Gruppen eher dazu, einen Raum nicht für sich zu beanspruchen, wenn bereits männliche Sportteilnehmer diesen besetzen (Dietrich, 2001). Löw (2001) schlussfolgert, dass Mädchen nicht auf die Rollen vorbereitet werden, die eine räumliche Kompetenz erfordert. Dietrich (2001) unterstützt diese Auffassung und vermutet, dass der Verlass auf Körper als Machterfahrung vielen Mädchen scheinbar fehle, was auch das Fernbleiben von Risikoerfahrungen sowie von Konflikt- und Durchsetzungsfreude erklären würde. Die mangelnde räumliche Kompetenz von Mädchen kann darüber hinaus ebenfalls auf die stärkere Verinselung von Mädchen zurückgeführt werden. Mädchen wird eher von Seiten der Eltern verboten, alleine zum Sportverein zu fahren. Die verstärkte Beaufsichtigung der Eltern, die ihre Töchter vor sexuellen Grenzverletzungen schützen wollen, ist in den meisten Fällen der Grund dieses Verhaltens (Sobiech, 2002). Eine weitere Erklärung für die schwache Ausprägung der räumlichen Kompetenz könnte sein, dass Mädchen sich in der Pubertät aus freien Zügen, mitunter aufgrund der Verunsicherung über den heranwachsenden Körper, zurückziehen. Löw (2001) argumentiert, dass sich die daraus resultierenden fehlenden Kompetenzen auch in der späteren Berufswahl niederschlagen können und ergo keine Chancengleichheit zwischen Männern und Frauen gewährleistet wäre.

Zusammenfassend lässt sich festhalten, dass Bewegungsräume die Möglichkeiten und Grenzen der Bewegungssozialisation mitdefinieren (Burrmann, 2008). Entscheidend in diesem Prozess der Aneignung und Konstruktion sind die Lebensbedingungen der Familie, die ökonomisch und kulturell verfügbaren Ressourcen der sozialen Klasse und die Geschlechtszuge- 
hörigkeit, da diese die Möglichkeiten und Grenzen des Handelns, Wahrnehmens und Denkens bedingen.

\subsection{Fazit und Diskussion}

Es wird zunehmend gefordert, dass die Bedeutung von öffentlichem Raum als wichtiger Bildungsort für Kinder wiedererkannt und bei stadtplanerischen Aspekten mitbeachtet wird (Deinet, 2010). Breckner (2010) argumentiert, dass das Thema Bewegung in Programmen zur sozialen Stadtteilentwicklung keine systematische Rolle spielt. Die Planung von Bewegungsräumen erfolgt noch zu selten unter Berücksichtigung der verschiedenen Nutzungsmilieus. Zudem kritisiert Breckner (2010), dass bei Planungsprozessen zu häufig eine reine Objektperspektive dominiert, bei der Spielräume lediglich um der Existenz Willen gebaut werden, ohne dass diese einer bewegungsspezifischen Eignungsprüfung unterzogen würden oder die Nutzergruppen bei der Bauplanung mitwirken könnten. Breckner (2010) fordert daher, nicht nur die Interessen der Nutzergruppen in stadtplanerische Entscheidungen einfließen zu lassen, sondern auch, dass die Bearbeitung solcher Angelegenheiten durch Fachleute aus Pädagogik, Medizin und Bewegungswissenschaften unterstützt wird. Denn obwohl ein bedeutender Zusammenhang zwischen Raumaneignung und Sport besteht, werden Sportwissenschaftler und Fachleute aus dem Gesundheitswesen bei der Stadtplanung selten zu Rate gezogen.

Um wieder mehr Platz für Bewegungsvielfalt und informelle Sportaktivitäten zu schaffen, wäre ein Lösungsansatz die Auflösung monofunktionaler Räume zugunsten multifunktionaler Bewegungsflächen, auf denen sich verschiedene Nutzergruppen eine gemeinsame Fläche teilen könnten. Tatsächlich werden in der Landschaftsarchitektur öffentliche Freiräume zunehmend so gestaltet, dass sie sich bspw. auch zum Tanzen, Skaten oder Ballspielen eignen (Breckner, 2010). Das Konzept des sogenannten "Shared Space" scheint u.a. in der Stadt Freiburg zu fruchten. Dort entstehen bspw. Fahrradstraßen, auf denen Fahrradfahrer sich die Straße mit Autofahrern teilen oder Flächen wie etwa ein trocken gelegter Brunnen vor dem Mensagarten, der im Sommer als Skateboard- und Tanzfläche genutzt werden darf. Angesichts der sozialstrukturellen und milieuspezifischen Heterogenität von Nutzergruppen müssen öffentliche Bewegungsräume unterschiedlichen sozialen, sachlichen, kulturellen und wirtschaftlichen Anforderungen genügen (Breckner, 2010). Zur Gestaltung nachhaltiger Bewegungsräume schlägt Breckner (2010) daher vor, Qualitätsstandards für öffentliche Bewe- 
gungsräume zu erstellen, die auf fachlichen Ressourcen in unterschiedlichen Disziplinen wie der Bewegungswissenschaft und auf Nutzungserfahrungen basieren. Es käme darauf an, mit Hilfe empirischer Wissensgrundlagen Bewegungskulturen und ihre räumlichen Voraussetzungen wahrzunehmen und zu analysieren, um auf dieser Basis für jeden öffentlichen Raum das ihm angemessene Bewegungsangebot zu konzipieren und umzusetzen (ebd.).

Bewegungsräume sind attraktiv, wenn sie eine Aneignung für unterschiedliche Nutzergruppen ermöglich - und dies zu allen Tages- und Jahreszeiten sowie zu mehreren Zwecken (ebd.). Durch einen stadtplanerischen Wandel hin zu multifunktionellen öffentlichen Räumen, die die Heterogenität der Nutzungsgruppen berücksichtigen, könnten soziale Räume wieder geöffnet und als Lernfelder für Kinder und Jugendliche erschlossen werden. Im Hinblick auf die Unterschiede in geschlechtertypischen Sozialisationsprozessen zwischen Jungen und Mädchen, die sich häufig zum Nachteil der Mädchen auswirken, sollte der Fokus noch mehr auf Projekte gelegt werden, die Mädchen Anregung zur Raumaneignung geben und dadurch die Eigenständigkeit der Mädchen fördern.

Ein Projekt, das sich vor allem der Sportförderung von Mädchen mit Migrationshintergrund widmet, ist das Projekt „Kick for Girls“ des Instituts für Sportwissenschaften der Universität Freiburg. Über Fußball-AGs für Mädchen in Grund- und Hauptschulen soll den Mädchen Raum gegeben werden, den männerdominierenden Fußball für sich zu entdecken und die Freude am Fußball ohne geschlechtsspezifischen Konkurrenzdruck zu entwickeln. Der Erfolg des Projekt liegt auch darin, dass ein Lernen im geschützten Raum gewährleistet wird, so dass die Mädchen ihrer Sportaktivität nachgehen können, ohne dass die Eltern Angst vor sexuellen Grenzüberschreitungen haben müssen und diese das Projekt daher unterstützen. „Kick for Girls" bietet den Mädchen folglich eine Chance, anderen kulturellen Werten zu begegnen und bietet der zunehmenden Verhäuslichung junger Mädchen eine gesicherte Alternative.

\section{Die Ganztagsschule in Deutschland 2012/2013}

Bevor eine umfassende Bewertung und Handlungsempfehlung hinsichtlich des Ganztagsschulkonzepts im letzten Kapitel dieser Abschlussarbeit erfolgt, sollen vorher die aktuellen diesbezüglichen Statistiken eruiert werden. Seitdem der Ausbau der Ganztagsschule nach der PISA-Offenbarung von 2001 politisch gefordert und finanziell unterstützt wurde, führte dieses Vorhaben zu einer der „rasant[est]en bildungspolitischen Veränderungen in der 
Schullandschaft" (Derecik, Kaufmann \& Neuber, 2013, S. 24). Doch wie sieht diese Landschaft heute aus und was ist aus ihr geworden?

\subsection{Entwicklungstendenzen der Ganztagsschulen in Deutschland}

Die von der Bertelsmann-Stiftung initiierte Studie geht dem Versuch nach, eine Analyse über den Ausbau und Entwicklung der Ganztagsschulen in Deutschland vorzunehmen. Dabei sollen erhobene Daten mehr Aufschluss liefern, um diese „Erkenntnislücke bzgl. des Ausbaustandes zu schließen“ (Klemm, 2013, S. 4). Die Studie veröffentlichte 2013 bzw. 2014, dass im Schuljahr 2012/2013 insgesamt 2,4 Millionen bzw. 32,3 \% aller SuS in Deutschland eine private oder öffentliche Primar- oder Sekundarstufe I innerhalb einer Ganztagsschule besuchten. Dies bedeutete einen Anstieg um 1,6 \% im Vergleich zum Vorjahr, wenngleich auch diese Zahlen international gesehen vergleichsweise gering einzustufen sind (ebd.).

Obwohl die Daten der Bundesrepublik sich verhältnismäßig überschaubar gestalten, so kann dennoch eine Verdreifachung des Ganztagsbetriebes von 2002 bis 2012 verzeichnet werden. In prozentualen Zahlen drückt sich dieser Anstieg von 9,8 \% auf 32,3\% aus. Geht es jedoch um die Befragung der Eltern, so wünschten sich diese im Jahr 2012 einen Ausbau der Ganztagsschulen von bis zu 70 \% (Klemm, 2014).

Im Schuljahr 2012/2013 waren über die Hälfte aller privaten und öffentlichen Verwaltungseinheiten der Primar- und Sekundarschulen Ganztagsschulen. Auch hier ist zu vermerken, dass ein Kontrast von Sachsen mit 96,7 \% zu bspw. Baden- Württemberg 30,5 \% nicht zu verkennen ist. Damit gab es im Schuljahr 2012/2013 einen Anstieg an Ganztagsschulen um 1,6 $\%$, d.h. von 54,3\% auf 55,9\% Verwaltungseinheiten (ebd.).

Auf einer Deutschlandkarte lässt sich der Anteil der Verwaltungseinheiten mit Ganztagsbetrieb an allen Verwaltungseinheiten in den Schuljahren 2011/12 und 2012/13 und auf die einzelnen Bundesländer bezogen, folgendermaßen darstellen: 


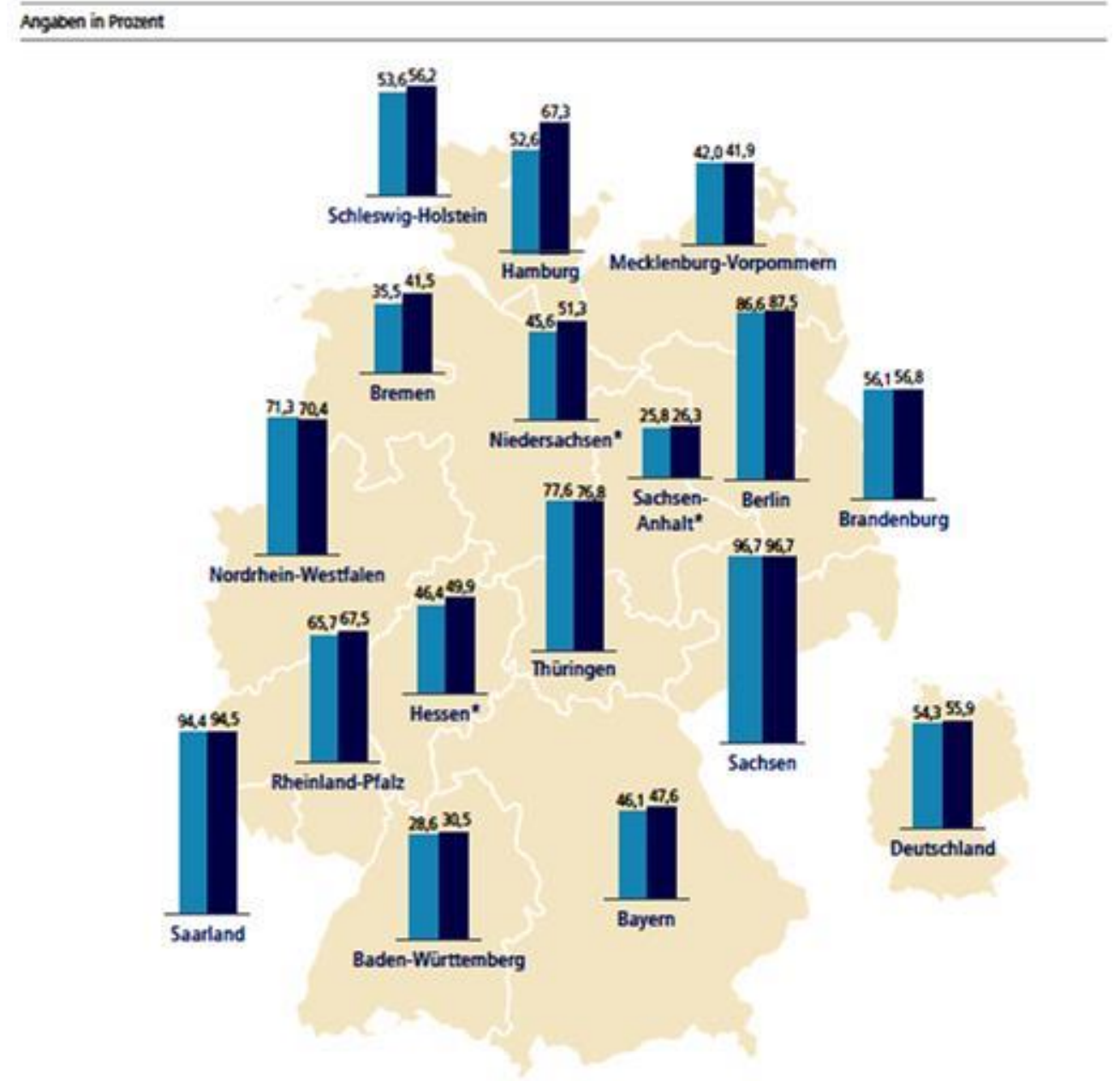

\section{1/12 2012/13}

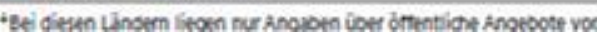

Abb. 9: Anteil der Verwaltungseinheiten mit Ganztagsbetrieb an allen Verwaltungseinheiten - Schuljahre 2011/12 und 2012/13 (Primar- und Sekundarstufe I in privater und öffentlicher Trägerschaft (Quelle: Klemm, 2014, S. 13)

Erörtert man die Teilnahmequote der SuS von 32,3\%, so legt die Studie offen, dass die Verteilungsquote der Ganztagschulen innerhalb Deutschlands sich recht heterogen verhält. So beziffert sich in Bayern die Nutzung des Ganztagsangebots der SuS auf 12,4 \%, während in Sachsen sich das Angebot auf 79,1 \% beläuft. Davon wiederrum besuchen in Deutschland nur $14,4 \%$ der SuS die gebundene und $18,0 \%$ die offene Ganztagesform.

Ebenfalls zeigt sich hier ein Gefälle innerhalb der Bundesländer: Derweil in Sachsen 29,3\% aller SuS von der ersten bis zehnten Klasse die gebundene bzw. 49,9 \% die offene Ganztagsschule besuchen, sind in Schleswig-Holstein gerade 4,6\%, in einer gebundenen und $19,2 \%$ in einer offenen Ganztagsschule untergebracht (Klemm, 2014). 


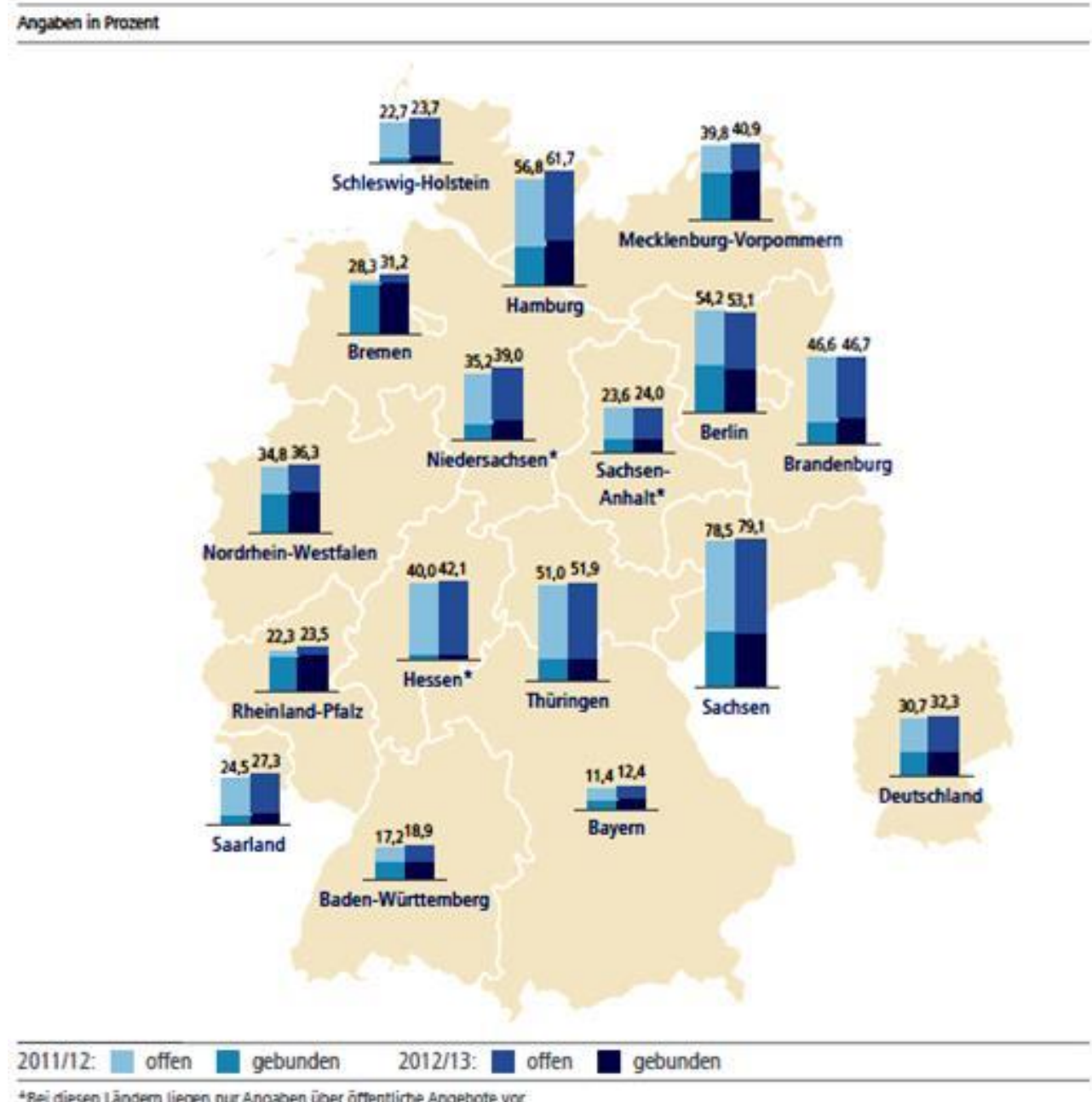

Abb. 10: Anteil der Schüler im Ganztagsschulbetrieb nach Angebotsform - Schuljahre 2011/12 und 2012/13 (Primar- und Sekundarstufe I in privater und öffentlicher Trägerschaft) (Quelle: Klemm, 2014, S. 15)

Nicht zu vernachlässigen sind diejenigen SuS, die an einigen Ganztagsschulen die Ganztagsangebote nicht wahrnehmen. Zum einen wurde von Seiten der SuS nicht der Wunsch geäußert, diese Angebote wahrzunehmen und zum anderen gab es oftmals nicht genügend Platzkapazitäten, um die an den Angeboten interessierten SuS mitaufzunehmen. Es muss zukünftig berücksichtigt werden, dass auch diese SuS einen erheblichen Einfluss auf die gesamte Teilnahmequote haben (Klemm, 2014.)

\subsection{Ausbauperspektiven von Ganztagsschulen in Deutschland}

Laut einer im Jahr 2012 durchgeführten JAKO-O-Bildungsstudie mit einer Umfrage an Eltern beträgt der Bedarf von Ganztagsschulplätzen umgerechnet 70 \%. Davon bevorzugten 38 \% der Eltern eine offene und 32\% eine gebundene Ganztagsschulform (Killmus \& Tillman, 2012). Der Grund für diese Ergebnisse ist mit großer Wahrscheinlichkeit der arbeitsbezogene 
Beschäftigungswunsch der Eltern. Denn erst durch die Betreuung der Kinder besteht die Gewährleistung, einer Arbeit in Vollzeit nachgehen zu können (Klemm, 2013).

Der momentane Ausbau der Ganztagsschulen verläuft allerdings, entgegengesetzt dem Wunsch mancher Eltern, deutlich eingeschränkter. So betrug der Anstieg der Ganztagsschulen von 2009 bis 2012 lediglich 1,3 \%. Nach derzeitigem Stand bedeutet dies, dass bis 2020 nur für 46,6 \% aller deutscher SuS ein Ganztagsschulplatz zur Verfügung stünde. Wenn jedoch die von Seiten der Eltern gewünschte 70 \%-Marke bis zu diesem Zeitpunkt erreicht werden sollte, dann müsste ein utopisch wirkender jährlicher Ausbau von mind. 4,3 \% erfolgen. Wären 100 \% das angestrebte Ziel, so müsste ein jährlicher Ausbau von 8,0 \%, (je nach Bundesland) umgesetzt werden (Klemm, 2014).

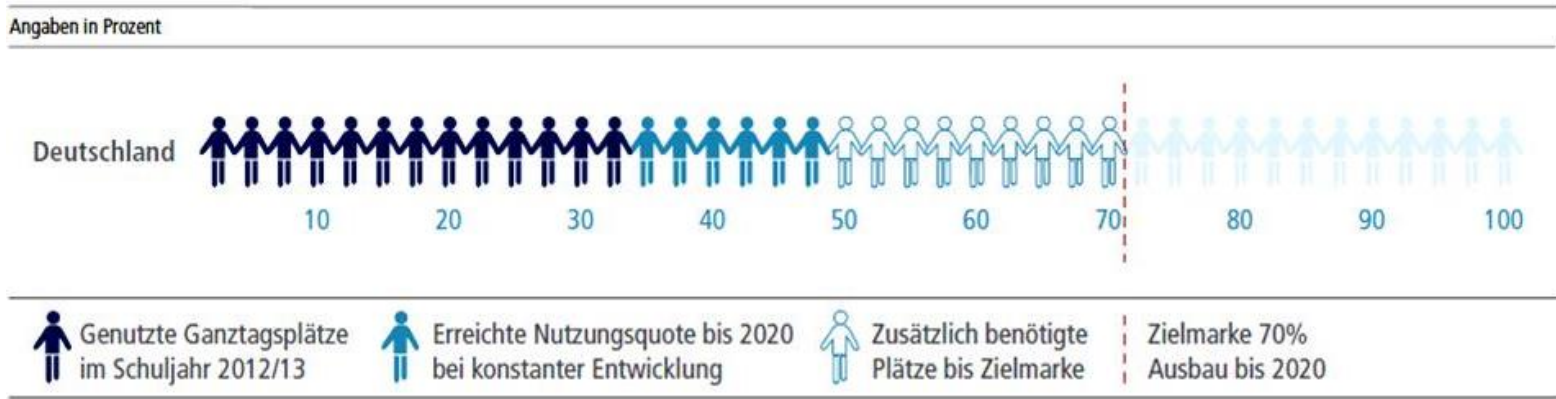

Abb. 11: Entwicklung der Ganztagsschulbeteiligung bis 2020/21 in Deutschland (Quelle: Klemm, 2014, S. 20)

Diese Zahlen sind im Jahr 2014 im Auftrag der Bertelsmann-Studie veröffentlicht worden. Im selben Jahr fanden die ersten Flüchtlingswellen in der Bundesrepublik Deutschland statt, deren Zahlen für die damalige Erhebung noch gar nicht mitberücksichtigt werden konnten. So ist zu bedenken, dass Migrationskinder und deren Eltern insbesondere nach der Genehmigung des Aufenthaltsrechts in einem besonderen Maß von einer Ganztagsschule profitieren könnten. Zum einen wäre das der erste Schritt, die Kinder zur Integration zu begleiten und zum anderen würde den Eltern die Eingliederung in die Gesellschaft durch etwaige Sprachkurse erleichtert und ein späterer Zugang zu Erwerbstätigkeit ermöglicht werden. Eine Erhöhung des Ausbautempos wäre demnach dringend zu empfehlen.

\section{Schlussbetrachtung}

Beim Symposium an der Südbadischen Sportschule in Steinbach 2007 erläuterte zur Thematik „Bewegung, Spiel und Sport in der Ganztagsschule“ der damalige Kultusminister Helmut Rau die Ganztagsschule aus bildungspolitischer Sicht folgendermaßen: 
„Ganztagschule ist etwas anderes als die verlängerte Regelschule. ,Mehr vom Gleichen' wie bisher in der Regelschule, und das ist dann Ganztagsschule - da würden wir einen großen Fehler begehen. Wir müssen bei guten Ganztagsschulkonzepten darauf achten, dass sich die Abläufe verändern. Es steht mehr Zeit zur Verfügung, um die gleichen schulischen Ziele zu erreichen, und wir müssen darauf achten, dass man die Zeit sinnvoll gestaltet und sinnvoll nutzt. Wir sprechen von einer Rhythmisierung der Ganztagsschulen, die größere Unterrichtsblöcke und größere Pausenzeiten beinhaltet - Chancen übrigens, sich nicht nur in einem Jugendbegleiter- Programm, sondern auch im Schulalltag regelmäßig angeleitet zu bewegen. Ganztagsschulen bieten zudem die Chancen, dass Kinder, deren Alltagsgewohnheiten sich aufzulösen beginnen oder in Gewohnheiten überzugehen, die für ihre Entwicklung nicht hilfreich sind, dass Kinder diese Alltagsgewohnheiten in der Schule wieder entwickeln können.“ (S. 18)

Die Rede des Ministers erweckt den Eindruck eines recht zwiespältigen Bildes. Zum einen enthält sie Kritik und den Aufruf zur Veränderung des Bisherigen und zum anderen werden dennoch die Vorzüge und Chancen aufgezeigt, welche eine Ganztagsschule leisten könnte. Diese Schlussbetrachtung versucht sich in diesen Tenor einzubetten, um die Herausforderungen dieser Schulform zu erörtern und wenn möglich einen Ausblick zu schaffen.

Die Wichtigkeit von Bewegung, Spiel und Sport und deren Implementierung in den Ablauf eines Ganztagsschulplans ist evident und zugleich teilweise eine logistische bzw. planerische sowie pädagogische Herausforderung. Die Evidenz von der Wichtigkeit eines bewegungsmotivierten Lebens ist hinreichend durch zahlreiche Studien belegt (vgl. u.a. Bös, 2003; Lampert et. al, 2007; Opper et. al, 2007). Obwohl bekannt ist, dass körperliche und sportliche Aktivität äußerst positive Effekte hervorrufen kann, so wird ihre Bedeutung häufig verkannt. Neben dem biophysiologischen stärkenden und schützenden Einfluss den der Sport innehat, fördert Bewegung auch andere Bereiche, wie bspw. die, der psychischen, kognitiven und sozialen Entwicklung. Und trotz dieses Wissens und der erläuterten sowie deutschlandweit bekannten Forschungsgrundlage besteht ein Bewegungsdefizit der SuS in Deutschland.

Die Ganztagsschule kann insbesondere durch das größere Zeitvolumen diesem Missstand entgegentreten und mehr Bewegungsmöglichkeiten anbieten. Die Schule ist in dieser Hinsicht jedoch sehr darauf angewiesen, ihre Institution für außerschulische Partner zu öffnen, um die Bewegungssituation zu verbessern. Wenn es dann zu einer Kooperation mit einem 
Verein kommt, so ist es nicht selten die Leistung der Sportlehrkräfte, die häufig den (privaten) Kontakt und folglich eine Zusammenarbeit ermöglichen. Das bedeutet, dass ein zusätzliches Eigenengagement und ein weiterer Zeitaufwand von den Sportlehrern/innen erforderlich wäre, damit etwaige Ansprüche und die genannten Ziele einer fruchtbaren Kooperation mit weiteren Partnern umgesetzt werden können.

Des Weiteren ist momentan die Wahrscheinlichkeit groß, dass Sport-AGs und weitere Angebote von Seiten der Schule in Ganztageseinrichtungen gar nicht zustande kommen können, da es sowohl die räumlichen, als auch die personellen Kapazitäten nicht zulassen (Klemm, 2014). Mit dem Angebot von Sport und Bewegung entsteht demnach simultan die Frage nach der Verfügbarkeit von Räumlichkeiten und personellen Ressourcen. Ergo müsste das Ziel formuliert werden, ohne weite Wegstrecken (andere Hallen bzw. Sportanlagen) und mit Hilfe von zusätzlichen ehrenamtlichen Übungsleitern/innen bzw. Lehrern/innen ein breites Sportangebot für Kinder und Jugendliche anbieten zu können. Die derzeitigen infrastrukturellen Gegebenheiten an deutschen Schulen sowie die personell benötigten Ressourcen sind für derartige Pläne jedoch bei weitem nicht gegeben. Auch für die Vereine ist es nahezu unmöglich, dass am Nachmittag ein/e jeweilige/r Übungsleiter/in zur Verfügung gestellt werden kann. Im Zusammenhang Schule und Verein gestaltet sich eine gemeinsame Zusammenarbeit oftmals auch als schwierig, da die unterschiedlichen Zielsetzungen der Schulen (pädagogische Bildung und Erziehung) und der Sportvereine (Leistungsdenken) aufeinanderprallen. Um dieser Interessensspaltung zu entgehen, wäre das vorgeschlagene integrative Modell von Laging et al. (2006), die sogenannte „Kooperation durch Inklusion“, mit hoher Wahrscheinlichkeit die am erfolgversprechendste Alternative. Die im Vordergrund stehende Maxime „Pädagogik von Bewegung“ könnte durch einen gemeinsamen Konzeptentwurf die grundlegende Basis für eine erfolgreiche Zusammenarbeit zwischen Schule und Verein darstellen. Dieses Konzept bietet weitaus mehr an als die vorgestellten additiven Modelle „Sport am Nachmittag“ bzw. „Sport im Ganztag“, denn in der sogenannten „Bewegten Schule“ würde ein Miteinander im Vordergrund stehen, wodurch beide Kooperationspartner gegenseitig voneinander profitieren könnten und was noch viel wichtiger ist: Die SuS würden durch dieses Konzept am meisten lernen und wären am besten versorgt.

Allerdings sind diese Modelle für offene Ganztagsschulen gedacht, da diese im Verhältnis zu gebundenen deutlich häufiger besucht werden. Offene Ganztagsschulen bieten eine freiwillige Teilnahme an den jeweiligen Angeboten. Dieses Vorgehen begrüßen auch die Vereine, weil ihre potenziellen Vereinsmitglieder dadurch nicht an die gebundenen Schulen „verlo- 
ren" gehen würden. Die Freiheit der SuS, wählen zu können, an welchen Tagen sie die Nachmittagsangebote von Seiten der Schule wahrnehmen möchten, wäre folglich gegeben. Hingegen sehen einige Eltern mit der gebundenen Form eine staatliche Einmischung in deren Erziehungseinfluss. Zudem bemängeln sie die die Einschränkung der Freizeit von Kindern und Jugendlichen in gebundenen Ganztagsschulen. Es stellt sich jedoch die Frage, welche der beiden Ganztagsformen in ihrer Qualität empfehlenswerter sind. Wie eben erwähnt, bietet die offene Form den SuS wesentlich mehr Freiheit und hat dadurch nicht selten größere Angebotsspektren. Doch die Erhebungen von Klemm (2013) weisen darauf hin, „dass positive Effekte bei Schülern sich eher in der gebundenen Form der Ganztagsschule zeigen können“ (S. 6). Begründet wird diese Annahme, dass Untersuchungen gezeigt haben, „dass Kinder und Jugendliche, die regelmäßig an guten Ganztagsangeboten teilnehmen, bessere Lernerfolge erzielen. Gebundene Ganztagsschulen ermöglichen [diese] individuellere Förderung und eröffnen mehr Lernchancen für die Schüler" (Klemm, 2014, S. 4).

Nichtsdestotrotz sind noch erhebliche Defizite und Baustellen bzgl. beider Ganztagsschulformen im Allgemeinen zu verzeichnen. Weitere Studien belegten, dass SuS der Ganztagsschule gegenüber SuS der Halbtagsschule keine verbesserten kognitiven Fähigkeiten durch gezielte Förderungen aufwiesen (Klemm, 2013). In den Grundschulen wurden zumindest bessere Lesefähigkeiten der Ganztags-SuS gegenüber der Halbtags-SuS beobachtet (Bellin \& Tamke 2010). Steiner (2011) entdeckte, dass sich in den Klassenstufen 5 bis 9 die Gefahr verringerte, die Klasse wiederholen zu müssen. Kuhn und Fischer (2011) eruierten eine Verbesserung der Schulnoten bei SuS, die kontinuierlich den Ganztagsunterricht besuchten. Killus und Tillmann (2012) zeigten auf, dass sich die Situation einiger Eltern von Ganztags-SuS verbessert habe, da diese im Vergleich zu Eltern von Halbtags-SuS deutlich weniger Arbeitsaufwand in der Hausaufgabenkontrolle oder Klassenarbeitsvorbereitung leisten müssten. Die Inanspruchnahme von Nachhilfe ist jedoch bei beiden Schulformen gleich hoch, obgleich diese Leistung eigentlich von den Ganztagesschulen abgedeckt werden sollte (ebd.).

Die Ergiebigkeit der Ganztagsschule steht laut derzeitiger Studienlage in enger Korrelation mit dem kontinuierlichen Besuch des Ganztagsunterricht. Folglich rät die allgemeine Forschung eher von einer offenen Form ab, da diese den SuS einen größeren individuellen Entscheidungsraum lässt und ein kontinuierlicher Besuch des Unterrichts aller SuS nicht gewährleistet werden kann. Als weiteres Pro-Argument für die gebundene Form wurde anhand der erläuterten Studien festgestellt, dass Kinder und Jugendliche aus sozial schwächeren Familien oder mit Migrationshintergrund seltener in diesen Förderbereich in Schulen mit offener 
Ganztagseinrichtung eintreten, als dies in der gebundenen Form der Fall ist. Dennoch ist ein Plädoyer für weitere Studien gegeben, damit diese Vermutungen verifiziert werden können (Klemm, 2014).

Wenn SuS mit Migrationshintergrund und niedrigem Sozialstatus die zahlreichen Schulangebote nicht wahrnehmen, so verschließen sich ihnen Türen in zweierlei Hinsicht. Einerseits wird dadurch die Förderung kognitiver und integrativer Kompetenzen eingeschränkt und andererseits wird die Bewegungsvielfalt, welche u.a. zur Erhaltung der Gesundheit beiträgt, limitiert. Doch genau diese Personengruppen sollen u.a. durch das Ganztagsschulsystem aufgefangen werden. Dennoch muss auch die Kritik beachtet werden, dass eine staatliche Institution nicht ausschließlich den Tagesablauf der Kinder und Jugendlichen bestimmen darf, wie es bei Ganztagsschulen durchaus der Fall wäre. Es kann tatsächlich der Eindruck entstehen, dass durch dieses Schulkonzept die Eigenbestimmung des Lebens junger Menschen erheblich beschnitten und auf die Fremdbestimmung Erwachsener reduziert wird. Aus dieser Sichtweise ist wohl eher die Wahl einer offenen Ganztagsschulform ratsam, da den Kindern und Jugendlichen hier mehr Rechte auf Eigenbestimmung eingeräumt werden.

Meines Erachtens erscheint anhand der Studienergebnisse und der erwähnten Kritikpunkte eine gebundene Form mit deutlich größerer Partizipation sowie erhöhtem Mitspracherecht seitens der SuS am sinnvollsten. So könnte in erster Linie auch Sozial-Benachteiligten die Chance offeriert werden, einen wesentlichen Beitrag unter der Begleitung von Fachpersonal ihrer eigenen „Stimme“ zu leisten und geltend zu machen. Innerhalb der Forschungsliteratur sehen die Experten einen wichtigen Bereich des Ganztagsschulbetriebs in der bereits angesprochenen Partizipation der SuS. Denn hier können sie sich miteinbringen, um z,B. an der Auswahl von Bewegungsangeboten mitzuwirken.

Mit dieser Integration der SuS hinsichtlich der Schulkultur sehen die Experten zudem eine höhere Erfolgswahrscheinlichkeit bzgl. der Teilnahme und der Motivation insbesondere bei körperlich-sportlichen Bewegungsangeboten. Die verstärkte Beteiligung der SuS an Schulprogrammen soll ihnen außerdem dazu verhelfen, in ihrem persönlichkeitsbildenden Entwicklungsprozess durch die eigene Gestaltung mitzuwirken. Dazu bietet sich der Bereich der Bewegung, des Spiels und Sports im Ganztag besonders gut an.

Derecik, Kaufmann und Neuber (2013) fassen die Möglichkeiten und Chancen des Sports in der Ganztagsschule folgendermaßen zusammen: 
„Im Gegensatz zum Sportunterricht können die Voraussetzungen, Interessen und Bedürfnisse der Kinder in Bewegungs-, Spiel- und Sportangeboten im Ganztag stärker berücksichtigt werden. Die pädagogischen Fachkräfte können sich besser auf die Spielideen der Kinder einlassen, sie können offene Situationen zum Experimentieren mit Bewegungsmöglichkeiten herstellen, und vor allem können die Kinder deutlich intensiver als im Unterricht ihre Bewegungs- Spiel- und Sportangebote selbst kreieren." (S. 19)

Eventuell spricht eine derartige Inklusion der SuS gerade die etwas außenstehende Schülerschaft an, die sich ansonsten eher zurückzieht und bietet so diesen SuS gleichzeitig eine Integrations- und Partizipationsmöglichkeit. Dennoch verweisen Derecik et al. (2013) darauf hin, dass in der Umsetzung und im „Know how“ immer noch Lücken bestehen (z.B. fehlende personale Ressourcen und Infrastruktur), die Ganztagsschulform jedoch obgleich ein großes Potenzial in sich birgt.

Wenngleich es noch kontroverse Auffassungen bzgl. der offenen und gebundenen Schulform gibt, so kann dennoch festgehalten werden, dass das Modell der „Bewegten Schule“ sicherlich eine Vielzahl an defizitären Bereichen an deutschen Schulen kompensieren könnte. Die in Kapitel 9 genannten acht Bausteine einer Bewegten Schule bieten mit den vorgeschlagenen Umsetzungsmöglichkeiten eine besondere Form der Bewegungsförderung innerhalb des Ganztagsunterrichts. Dadurch kann der Bewegungsmangel minimiert und individuelle Körpererfahrungen der SuS ungemein gefördert werden.

Des Weiteren können durch klug angelegte und auf Bewegungsfreude abgezielte Räume individuelle Lernkompetenzen unterstützt werden. Einer der wichtigsten Aspekte sehe ich in dem Vorhaben, die Schule als einen Bildungsort zu gestalten, der von den SuS nicht nur als Lehr- und Lernraum wahrgenommen wird, sondern in der Symbiose mit vielfältigen Bewegungsangeboten ein Raum für Kinder und Jugendliche, aber auch für Lehrerinnen und Lehrer darstellt, indem neben der reinen Wissensvermittlung, dem interkulturellen Austausch sowie der pädagogischen Förderung, auch Kritik und Unvollkommenheit existieren darf.

Selbstredend werden u.a. noch weitere Studien, Lehrerstellen, Fortbildungsmaßnamen und infrastrukturelle Maßnahmen benötigt, damit solide Verbesserungen des Ganztagsschulmodells in Deutschland erreicht werden können. Für eine wahrhaftige Realisierung dieser Ziele muss jedoch vor allem eine vehement bessere politisch-finanzielle Förderung von Bildung nicht nur angekündigt, sondern auch verwirklicht werden. 


\section{Literaturverzeichnis}

Albon, H.M., Hamlin, M.J. \& Ross, J.J. (2010). Secular trends and distributional changes in health and fitness performance variables of 10-14-year-old children in New Zealand between 1991 and 2003. British Journal of Sports Medicine, 44, 263-269.

Albrecht, C., Hanssen-Doosel, A., Bös, K., Schlenker, L., Schmidt, S., Wagner, M., Will, N. \& Worth, A. (2016). Motorische Leistungsfähigkeit von Kindern und Jugendlichen in Deutschland. Eine 6-Jahres-Kohortenstudie im Rahmen des Motorik-Moduls (MoMo). Sportwiss 2016 (46), 294-304.

Baacke, D. (1980). Der sozialökologische Ansatz zur Beschreibung und Erklärung des Verhaltens Jugendlicher. In: deutsche jugend (11), $493 \mathrm{ff}$.

Bai, D.L. \& Bertenthal, B.I. (1992). Locomotor status and the development of spatial search skills. Child Development, 63, 215-226.

Baumert, J., Klieme, E., Neubrand, M., Prenzel, M. \& Schiefele, U. (2001). PISA 2000: Basiskompetenzen von Schülerinnen und Schülern im internationalen Vergleich. Opladen: Leske + Budrich.

Bellin, N. \& Tamke, F. (2010). Bessere Leistungen durch Teilnahme am offenen Ganztagsbetrieb? Empirische Pädagogik, (24), 93-102.

Biddle, S.J.H., Gorely, T. \& Stensel, D.J. (2004). Health- enhancing physical activity and sedentary behaviour in children and adolescents. Journal of Sports Sciences, 22, 679-701.

Bös, K. (2001). Handbuch motorischer Tests. Göttingen: Hogrefe.

Bös, K., Opper, E. \& Woll, A. (2002). Fitness in der Grundschule - ausgewählte Ergebnisse. Haltung und Bewegung, 22, 5-20. 
Bös, K. (2003). Motorische Leistungsfähigkeit von Kindern und Jugendlichen. In: W. Schmidt, I. Hartmann-Tews \& W-D. Brettschneider (Hrsg.), Erster Deutscher Kinder- und Jugendsportbericht (S. 85-109). Schorndorf: Hofmann.

Bös, K., Oberger, J., Lämmle, L., Opper, E., Romahn, N., Tittlbach, S., Wagner, M., Woll, A., \& Worth, A. (2008). Motorische Leistungsfähigkeit von Kindern. In W. Schmidt (Hrsg.), Zweiter Deutscher Kinder- und Jugendsportbericht. Schwerpunkt: Kindheit (S. 136-157). Schorndorf: Hofmann.

Brandl-Bredenbeck, H.P. \& Brettschneider, W.-D. (2010). Kinder heute. Bewegungsmuffel, Fastfoodjunkies, Medienfreaks? Eine Lebensstilanalyse (Sportentwicklungen in Deutschland, 22). Aachen, Graz u.a: Meyer \& Meyer.

Breithecker, D. (1996). Bewegtes Sitzen - ist das kein Widerspruch? Grundschule, 28 (10), 2123.

Bundesministerium für Gesundheit und Soziale Sicherung (2005). Lebensverhältnisse in Deutschland. 2. Armuts- und Reichtumsbericht der Bundesregierung. Berlin: Eigenverlag.

Bundeszentrale für gesundheitliche Aufklärung (2003). Suchtprävention im Kinder- und Jugendsport. Theoretische Einordnung und Evaluation der Qualifizierungsinitiative „Kinder stark machen“. Eigenverlag, Köln.

Burdette, H.L. \& Whitaker, R.C. (2005). Resurrecting Free Play in Young Children: Looking Beyond Fitness and Fatness to Attention, Affiliation, and Affect. Archives of Pediatrics \& Adolescent Medicine, 159, 46-50.

Burrmann, U. (2008). Bewegungsräume und informelle Bewegungs-, Spiel- und Sportaktivitäten der Kinder. In W. Schmidt (Hrsg.), Zweiter Deutscher Kinder-und Jugendsportbericht. Schwerpunkt: Kindheit (Deutscher Kinder- und Jugendsportbericht (S. 391-408). Schorndorf: Hofmann. 
Calfas, K.J. \& Taylor, W.C. (1994). Effects of physical activity on psychological variables in adolescents. Pediatric Exercise Science, 6, 406-423.

Campbell, R., Starkey, F., Holliday, J., Audrey, S., Bloor, M., Parry-Langdon, N., Hughes, R. \& Moore, L. (2008). An informal school-based peerled intervention for smoking prevention in adolescence (ASSIST): a cluster randomised trial. Lancet, 371, 1595-1602.

Cavill, N., Biddle, S. \& Sallis, JF. (2001). Health enhancing physical activity for young people: Statements of the United Kingdom Expert Consensus Conference. Pediatric Exercise Science, $13,12-15$.

Chaddock, L., Erickson, K. I., Prakash, R. S., Kim, Js S., Voss, M. W., VanPatter, M. \& Kramer, A. F. (2010a). A neuroimaging investigation of the association between aerobic fitness, hippocampal volume and memory performance in preadolescent children. Brain Research, $1358,172-183$.

Colcomve, S. \& Kramer, A. F. (2003). Fitness effects on the cognitive function of older adults: A meta-analytic study. Psychological Science, 14, 125 - 130.

De Bock, F. (2012). Bewegungsförderung im Kindes- und Jugendalter. Geuter, G. \& Hollederer, A. (Hrsg.): Handbuch Bewegungsförderung und Gesundheit. (S. 131-152). Bern: Huber Verlag.

Deinet, U. (2005). Sozialräumliche Jugendarbeit : Grundlagen, Methoden und Praxiskonzepte. Wiesbaden: VS Verlag für Sozialwissenschaften.

Deinet, U. \& Reutlinger, C. (2004). "Aneignung" als Bildungskonzept der Sozialpädagogik. Beiträge zur Pädagogik des Kindes- und Jugendalters in Zeiten entgrenzter Lernorte (1. Aufl). Wiesbaden: VS Verlag für Sozialwissenschaften. 
Deinet, U. (2010). Informelle Bildung als Raumaneignung. In N. Neuber (Hrsg.), Informelles Lernen im Sport (S. 79-99): VS Verlag für Sozialwissenschaften.

Derecik, A. (2009). Informelles Lernen im Ganztag - eine sportpädagogische Studie zur sozialräumlichen Aneignung von Schulhöfen. In N. Neuber (Hrsg.), Informelles Lernen im Sport. Bewegung, Spiel und Sport in der Ganztagsbildung (S. 154-172). Wiesbaden: VS Verlag für Sozialwissenschaften.

Derecik, A., Kaufmann, N. \& Neuber, N. (2013). Partizipation in der offenen Ganztagsschule. Pädagogische Grundlagen und empirische Befunde zu Bewegungs-, Spiel- und Sportangeboten. Wiesbaden: Springer.

Dickhut, H.-H. (2000). Muskelphysiologie. In Ommo Gruppe (Hrsg.), Einführung in die Sportund Leistungsmedizin. Schorndorf. Hofmann.

Dietrich, K. \& Moegling, K. (2001). Spiel- und Bewegungsräume im Leben der Stadt. Sozialund erziehungswissenschaftliche Untersuchungen und Projekte. Butzbach-Griedel: AfraVerlag.

Dordel, S. (1998). Ätiologie und Symptomatik motorischer Defizite und Auffälligkeiten. In: Bundeszentrale für gesundheitliche Aufklärung BzGA (Hrsg.), Gesundheit von Kindern Epidemio- logische Grundlagen. Band 3: Forschung und Praxis in der Gesundheitsförderung ( S. 49-59) ). 2. Aufl. BZgA, Köln.

Dordel, S. (2000). Kindheit heute: Veränderte Lebensbedingungen=reduzierte motorische Leistungs- fähigkeit? Motorische Entwicklung und Leistungsfähigkeit im Wandel. Sportunterricht, 49, 341-349.

Eaton, W.O., Mckeen, N.A. \& Campbell, D.W. (2001). The Waxing and Waning of Movement: Implications for Psychological Development. Developmental Review, 21, 205-223. 
Ekeland, E., Heian, F. \& Hagen, K.B. (2005). Can exercise improve self esteem in children and young people? A systematic review of randomised controlled trials. British Journal of Sports Medicine, 39, 792-798.

Etnier, J. L. \& Chang, Y.-K. (2009). The effect of physical activity on executive function: A brief commentary on definitions, measurement issues, and the current state oft he literature. Journal of Sport \& Exercise Psychology, 31, $469-483$.

Erickson, K. I., Prakash, R. S., Voss, M. W., Chaddock, L., Hu, L., Morris, K. S. \& Kramer, A. F. (2009). Aerobic fitness is associated with hippocampal volume in elderly humans. Hippocampus, 19, $1030-1039$.

Fessler, N. \& Ziroli, S. (1997). Zusammenarbeit von Schule und Verein im Sport. Programme, Projekte und Perspektiven. Hoffmann: Schorndorf.

Fessler, N. (2003). Sport, Spiel und Bewegung in der Ganztagsschule: Freizeitgestaltung oder Schulprogramm. engagement - Zeitschrift für Erziehung und Schule, (2003) 3, 210-225.

Fessler, N. \& Stibbe, G. (2007). Standardisierung, Professionalisierung, Profilierung - Herausforderungen für den Schulsport. Hohengehren: Schneider.

Flade, A. \& Kustor-Hüttl, B. (1993). Mädchen in der Stadtplanung. Weinheim: Juventa.

Fuchs, R. (2003). Sport, Gesundheit und Public Health. Hogrefe, Göttingen.

Funke, J.; Heine, E. \& Schmerbitz, H. (1979). Initiativstunde. Erfahrungen in einer 6. Klasse der Bielefelder Laborschule. sportpädagogik, 3 (1), 36-39.

Gaschler, P. (1999, 2000, 2001). Motorik von Kindern und Jugendlichen heute - eine Generation von „Weicheiern, Schlaffis und Desinteresse“? (Teil 1, Teil2, Teil 3). Haltung und Bewegung, 19(3), 5-16; 20(1), 5-16; 21(1), 5-17. 
Geuter, G. \& Hollederer, A. (2012). Bewegungsförderung und Gesundheit. In G. Geuter \& A. Hollederer (Hrsg.), Handbuch Bewegungsförderung und Gesundheit (S. 9- 19). Bern: Huber.

Ginsburg, K.R., the Committee on Communications \& the Committee on Psychosocial Aspects of Child \& Fami- ly, Health (2007). The Importance of Play in Promoting Healthy Child Development and Maintaining Strong Parent-Child Bonds. Pediatrics, 119, 182191.

Grabs, R. (2004). Was Jugendverbände können, können nur Jugendverbände - Die Sportvereine und ihre Sportjugenden vor Herausforderungen durch die offene Ganztagsgrundschule. In E. Beckers, T. Schmidt-Millard (Hrsg.), Jenseits von Schule: Sportpädagogische Aufgaben in außerschulischen Feldern (S. 105-112). Butzbach-Griedel: Afra.

Hamer, M., Stamatakis, E. \& Mishra, G. (2009). Psychological distress, television viewing, and physical activity in children aged 4 to 12 years. Pediatrics, 123, 1263-1268.

Hansen, D.M., Larson, R.W. \& Dworkin, J.B. (2003). What Adolescents Learn in Organized Youth Activities: A Survey of Self-Reported Developmental Experiences. Journal of Research on Adolescence, 13, 25-55.

Helsper, W. (2001). Die sozialpädagogische Schule als Bildungsvision? Eine paradoxe Entparadoxierung. In P. Becker \& J. Schirp (Hrsg.), Jugendhilfe und Schule. Zwei Handlungsrationalitäten auf dem Weg zu einer? (S. 20-45). Münster: Votum.

Hernelahti, M., Kujala, U. \& Kaprio, J. (2004). Stability and change of volume and intensity of physical activity as predictors of hypertension. Scandinavian Journal of Public Health, 32, 303-309.

Hildebrandt-Stramann, R. (1999). Bewegte Schulkultur. Schulentwicklung in Bewegung. Butzbach-Griedel: Afra. 
Hillmann, C. H., Erickson, K.I. \& Kramer, A. F. (2008). Be smart, exercise your heart: exercise effects on brain and cognition. Nature Reviews Neuroscience, 9, 58-65.

Hillmann, C. H \& Schott, N. (2013). Der Zusammenhang von Fitness, kognitiver Leitungsfähigkeit und Gehirnzustand im Schulkindalter. Konsequenzen für die schulische Leistungsfähigkeit. Zeitschrift für Sportpsychologie, 20 (1), 33- 41.

Hoffmann, A., Brandt, R. \& Schlicht, W. (2006). Körperliche Bewegung. In: A. Lohaus, M. Jerusalem, J. Klein- Heßling (Hrsg.), Gesundheitsförderung im Kindes- und Jugendalter (S. 201-219). Göttingen: Hogrefe.

Holtappels, H.G. (2005). Ganztagsschulen entwickeln und gestalten - Zielorientierungen und Gestaltungsansätze. In K. Höhmann, H.G. Holtappels, I. Kamski \& T. Schnetzer (Hrsg.), Entwicklung und Organisation von Ganztagsschulen. Anregungen, Konzepte, Praxisbeispiele (S. 7-44).Dortmund: IFS-Verlag.

Holtappels, H.-G. (2007). Angebotsstruktur, Schülerteilnahme und Ausbaugrad ganztägiger Schulen. In H.-G. Holtappels, E. Klieme, T. Rauschenbach \& L. Stecher (Hrsg.), Ganztagsschule in Deutschland. Ergebnisse der Ausgangserhebung der 'Studie zur Entwicklung von Ganztagsschulen' (StEG). Weinheim: Juventa.

Holtappels, H.-G., Klieme, E., Rauschenbach, T. \& Stecher, L. (Hrsg.) (2007). Ganztagsschule in Deutschland. Ergebnisse der Ausgangserhebung der "Studie zur Entwicklung von Ganztagsschulen" (StEG). Weinheim: Juventa.

Holtappels, H.-G. (2009). Ganztagsschule und Schulentwicklung. Konzeptionen Steuerung und Entwicklungsprozesse. In F. Prüß, S. Kortas \& M. Schöpa (Hrsg.), Die Ganztagsschule: von der Theorie zur Praxis. Anforderungen und Perspektiven für Erziehungswissenschaft und Schulentwicklung (S. 111-136). Winheim: Juventa.

Holzkamp, K. (1983). Grundlegung der Psychologie. Frankfurt a.M./New York. 
Horstkemper, M. (2005). Standards. Vermessungspädagogik oder Antrieb zur Verbesserung der Bildungsqualität? Pädagogik, 57 (9), 6-9.

Höhmann, K., Holtappels, H. G. \& Schnetzer, T. (2004). Ganztagsschule. Konzeptionen, Forschungsbefunde, aktuelle Entwicklungen. In H.G. Holtappels, K. Klemm, H. Pfeiffer, H.G. Rolff \& R. Schulz-Zander (Hrsg.), Jahrbuch der Schulentwicklung. Band 13. Daten, Beispiele, Perspektiven Jahrbuch der Schulentwicklung (S. 253-289). Weinheim und München: Juventa.

Hunt, L.P., Shield, J.P., Cooper, A.R., Ness, A.R. \& Lawlor, D.A. (2011). Blood pressure in children in relation to relative body fat composition and cardio-respiratory fitness. International Journal of Pediatric Obesity, 6, 275-284.

Jacobs, J. (1992). The death and life of great American cities. New York.

Klaes, L., Rommel, A., Cosler, D. \& Zens, YCK. (2000). Bewegungsstatus von Kindern und Jugendlichen in Deutschland. Kurzfassung einer Untersuchung im S9 Auftrag des Deutschen Sportbundes und der AOK-Die Gesundheitskasse. Bonn: WIAD.

Klein, M., Fröhlich, M., \& Emrich, E. (2011). Sozialstatus, Sportpartizipation und sportmotorische Leistungsfähigkeit. Sport and Society, 8(1), 54-79.

Kleine, W. (2003). Tausend gelebte Kindertage. Sport und Bewegung im Alltag der Kinder. Weinheim: Juventa.

Killus, D. \& Tillmann, K.- J.,(2012) (Hrsg.). Eltern ziehen Bilanz. 2. JAKO-O Bildungsstudie. Münster 2012.

Klemm, K. (2013). Ganztagsschulen in Deutschland - eine bildungsstatistische Analyse. Gutachten im Auftrag der Bertelsmann Stiftung. Gütersloh. 
Klemm, K. (2014): Im Auftrag der Bertelsmann Stiftung - Ganztagsschulen in Deutschland: Die Ausbaudynamik ist erlahmt. Gütersloh. Verfügbar unter https://www.bertelsmannstiftung.de/fileadmin/files/BSt/Publikationen/GrauePublikationen/Studie_IB_Ganztags schulen_in_Deutschland_Ausbaudynamik_erlahmt_2014.pdf [12.08.2017].

Klieme, E., Holtappels, H.-G., Rauschenbach, T. \& Stecher, L. (2007). Ganztagsschule in Deutschland, Bilanz und Perspektive. In H.-G. Holtappels, E. Klieme, T. Rauschenbach \& L. Stecher (Hrsg.), Ganztagsschule in Deutschland. Ergebnisse der Ausgangserhebung der 'Studie zur Entwicklung von Ganztagsschulen' (StEG) (S. 354-381). Weinheim: Juventa.

KMK (Kultusministerkonferenz) (2002). PISA 2000 - Zentrale Handlungsfelder. Zusammenfassende Darstellung der laufenden und geplanten Maßnahmen in den Ländern. Verfügbar unter www.kmk.org/fileadmin/pdf/Presse+UndAktuelles/2002/massnahmen.pdf [03.08.2017]

KMK (Kultusministerkonferenz) (2006). Kommission für Statistik: Definitionenkatalog zu Schulstatistik. Verfügbar unter http://www.kmk.org/fileadmin/Dateien/pdf/Statistik/ Defkat2011.pdf [12.08.2017]

KMK (Kultusministerkonferenz). Kommission für Statistik: Definitionenkatalog zur Schulstatistik. Verfügbar unter www.kmk.org/fileadmin/pdf/Statistik/KomStat/Defkat2011.pdf. [03.08.2017]

Kretschmer, J \& Giewald, C. (2001). Veränderte Kindheit - veränderter Schulsport? Sportunterricht, 50, 36-42.

Kuhn, H.-P. \& Fischer. N. (2011). Entwicklung der Schulnoten in der Ganztagsschule. Ein- flüsse der Ganztagsteilnahme und der Angebotsqualität. In N. Fischer, H.- G. Holtappels, E. Klieme, T. Rauschenbach, L. Stecher \& I. Züchner (Hrsg.), Ganztagsschule: Entwicklung, Qualität und Wirkungen (S. 207-226.). Weinheim. 
Kurth, B.-M. (2007). Der Kinder- und Jugendgesundheitssurvey (KiGGS): Ein Überblick über Planung, Durchführung und Ergebnisse unter Berücksichtigung von Aspekten eines Qualitätsmanagements. Bundesgesundheitsblatt Gesundheitsforschung Gesundheitsschutz. $50,533-546$.

Laging, R. (1993). Bewegung in die Schule! Die Grundschulzeitschrift, 70 (7), 8-15.

Laging, R. \& Klupsch-Sahlmann, R. (2001). Schulen in Bewegung. sportpädagogik, 25 (2), 410.

Laging, R. (2006). Bewegung, Spiel und Sport in der Ganztagsschule - Bedeutung für Lernen, Erziehung und Entwicklung. Vortrag auf dem Ganztagsschulkongress in Berlin am 22. 9. 2006. Verfügbar unter http://www.ganztaegig-lernen.de/media/bewegung-lernenentwicklung-ganztag.pdf [25.07.2017].

Laging, R. (2014).Bewegung, Spiel und Sport in der Ganztagsschule - Hintergründe und Forschungsstand. In R. Hildebrandt-Stramann, R. Laging \& J. Teubner (Hrsg. ), Bewegung und Sport in der Ganztagsschule-StuBBS: Ergebnisse der qualitativen Studie. Studie zur Entwicklung von Bewegung, Spiel und Sport in der Ganztagsschule (StuBBS) (S. 14-68). Hohengrehen: Schneider.

Laging, R. \& Hildebrandt-Stramann, R. (2006). Ganztagsschulen bewegungsorientiert entwickeln - Zwischen Schul- und Sportperspektive. Vortrag auf der Tagung der DGfEKommission Sportpädagogik am 24.11.2006. Verfügbar unter http://www.ganztaegiglernen.de/media/Material_1/karlsruhe_dgfe.pdf [11.08.2017].

Laging, R. \& Hildebrandt-Stramann, R. (2007). Ganztagsschulen bewegungsorientiert entwickeln - Zwischen Schul- und Sportperspektive. In N. Fessler \& G. Stibbe (Hrsg.), Standardisierung, Professionalisierung, Profilierung - Herausforderungen für den Schulsport. (S. 92-114). Hohengehren: Schneider. 
Lampert, T., Mensink, G. B. M., Romahn, N. \& Woll, A. (2007). Körperlich-sportliche Aktivität von Kindern und Jugendlichen in Deutschland. Ergebnisse des Kinder- und Jugendgesundheitssurveys (KiGGS). Robert Koch-Institut.

Lange, M., Kamtsiuris, P., Lange, C. et al. (2007). Messung soziodemographischer Merkmale im Kinder- und Jugendgesundheitssurvey (KiGGS) und ihre Bedeutung am Beispiel der Einschätzung des allgemeinen Gesundheitszustands. Bundesgesundheitsblatt Gesundheitsforschung Gesundheitsschutz 50, 578-589.

Leontjew, A. N. (1973). Probleme der Entwicklung des Psychischen. Frankfurt a. M.: Athenäum Fischer Taschenbuch Verlag.

Leppin, A., Kolip P. \& Hurrelmann K. (1996). Gesundheitsförderung in der Schule. Prävention A - Z Gesundheitsförderung, 19, 52-54.

Löw, M. (2001). Raumsoziologie (Suhrkamp-Taschenbuch Wissenschaft, 1506) (1. Aufl., Orig.-Ausg). Frankfurt am Main: Suhrkamp.

LSB NRW (2005). Sport im Ganztag 3. Schwerpunkte, Praxis, Perspektiven. Broschüre: Duisburg.

McDonald, N.C. (2007). Active transportation to school: trends among U.S. schoolchildren, 1969-2001. American Journal of Preventive Medicine, 32, 509-516.

Messner, R. (2004). Selbstreguliertes Lernen. Mehr Schüler-Selbständigkeit durch ein neues Konzept. Praxis Schule 5-10, 15 (5), 6-8.

Miedzinski, K. (2000). Die Bewegungsbaustelle (9. Aufl.). Dortmund: modernes lernen.

Naul, R. (2006). Sozialpädagogische und sportpädagogische Profession im Ganztag: Qualitätskriterien und Fortbildungsbausteine für Angebote mit Bewegung, Spiel und Sport. Expertise für das BLK-Verbundprojekt „Lernen für den GanzTag“. Broschüre: Universität Duisburg-Essen. 
Oelkers, J. (2003). Ganztagsschulen, Gesamtschulen und demokratische Schulkultur. Überlegungen zu Schulreform in Deutschland. Pädagogik, 55 (12), 36-40.

Opper, E., Worth, A., Wagner, M. \& Bös, K. (2007): Motorik-Modul (MoMo) im Rahmen des Kinder- und Jugendgesundheitssurveys (KiGGS) Motorische Leistungsfähigkeit und körperlich-sportliche Aktivität von Kindern und Jugendlichen in Deutschland. Bundesgesundheitsblatt Gesundheitsforschung Gesundheitsschutz (50), 879-888.

Pate, RR., Pratt, M. \& Blair, SN. (1995). Physical activity and public health. Journal of the American Medical Association, 273, 281-289.

Payne V.G. \& Isaacs, L. (2011). Human Motor Development: A Lifespan Approach, New York, McGraw Hill Higher Education.

Pellegrini, A.D. \& Smith, P.K. (1998). Physical activity play: the nature and function of a neglected aspect of playing. Child Development, 69, 577-598.

Pfeifer, K., (2003). Bewegungs- und Sporttherapie in der Rehabilitation. Public Health Forum, $41,18-19$.

PISA-Konsortium Deutschland (Hrsg.) (2004). PISA 2003. Der Bildungsstandart der Jugendlichen in Deutschland - Ergebnisse des zweiten internationalen Vergleichs. Münster. Waxmann.

PISA-Konsortium Deutschland (Hrsg.) (2007). PISA 2006. Die Ergebnisse der dritten internationalen Vergleichstudie. Münster. Waxmann.

Podlich, C. \& Kleine, W. (2003). Straßenkids-Straßen aus der Sicht der Kinder.

Prochaska, J., Sallis, J. \& Long B (2001). A physical activity screening measure for use with adolescents in primary care. Archives of Pediatrics and Adolescent Medicine, 155, 554559. 
Pühse, U. \& Illi, U. (1999). Bewegung und Sport im Lebensraum Schule. Schorndorf: Hofmann.

Quellenberg, H. (2007). Ganztagsschule im Spiegel der Statistik. In H.-G. Holtappels, E. Klieme, T. Rauschenbach \& L. Stecher (Hrsg.), Ganztagsschule in Deutschland. Ergebnisse der Ausgangserhebung der 'Studie zur Entwicklung von Ganztagsschulen' (StEG) (S. 1436). Weinheim: Juventa.

Rau, H. (2007). Ganztagsschulen aus bildungspolitischer Sicht - Chancen für Sportvereine und Verbände. In B. Seibel (Hrsg.), Bewegung, Spiel und Sport in der Ganztagsschule. Dokumentation eines Symposiums an der Südbadischen Sportschule Steinbach (S. 17-26). Schorndorf: Hofmann.

Reed, J.A., Einstein, G., Hahn, E., Hooker, S.P., Gross, V.P. \& Kravitz, J. (2010). Examining the impact of integrating physical activity on fluid intelligence and academic performance in an elementary school setting: a preliminary investigation. Journal of Physical Activity \& Health, 7, 343-351.

Röhner, Ch., Skischus, G. \& Thies, W. (Hrsg.) (1998). Was versuchen Versuchsschulen. Einblicke in die Reformschule Kassel. Baltmannsweiler: Schneider.

Sallis, JF. \& Owen, N. (1998). Physical activity and behavioural medicine. Sage, Thousand Oaks.

Schmidt, W. (2008). Zweiter Deutscher Kinder-und Jugendsportbericht. Schwerpunkt: Kindheit (Deutscher Kinder- und Jugendsportbericht). Schorndorf: Hofmann.

Schmidt, S., Will, N., \& Woll, A. (2016). Sportliche Aktivität deutscher Kinder und Jugendlicher im Verein und in der Schule. Die Motorik-Modul- Studie (MoMo). Sportunterricht, 65(8), 233-238. 
Sibley, B. A. \& Etnier, J. L. (2003). The relationship between physical activity and cognition in children: A meta-analysis. Pediatric Exersise Science, 15, 243 - 256.

Sobczyk, B. \& Landau, G. (2003). Das mobile Klassenzimmer: ein neuer Weg zur Entwicklungsund Bewegungsförderung von Grundschulkindern. Immenhausen bei Kassel: Prolog.

Sobiech, G. (2002). Gleicher Raum für alle? Aneignung von "Sport-Spiel-Räumen" im Spiegel von Klasse und Geschlecht. Forum Wissenschaft, 19(2), 14-18. Verfügbar unter http://www.bdwi.de/forum/archiv/archiv/441811.html. [28.07.2017]

Themenheft „Schulen in Bewegung" der Zeitschrift sportpädagogik, H. 2/2001.

Tillmann, K.-J. (2005). Ganztagsschule: die richtige Antwort auf PISA? In K. Höhmann, H.-G. Holtappels, I. Kaminski \& T. Schnetzer (Hrsg.), Entwicklung und Organisation von Ganztagsschulen. Anregungen, Konzepte, Praxisbeispiele (S. 45-58). Dortmund: IFS.

Timmons, B.W., Naylor, P.J. \& Pfeiffer, K.A. (2007). Physical activity for preschool children how much and how? Canadian Journal of Public Health, 98 (2 Suppl), 122-134.

US Department of health and human services (1996). Gesundheitsberichterstattung des Bundes - Körperliche Aktivität. In Statistisches Bundesamt und Robert Koch-Institut (Hrsg.). Verfügbar unter http://edoc.rki.de/documents/rki_fv/ren4T3cctjHcA/PDF/ 29BFVzVHbIM_45.pdf [12.08.2017]

Wilkin, T.J., Mallam, K.M., Metcalf, B.S., Jeffery, A.N. \& Voss, L.D. (2006). Variation in physical activity lies with the child, not his environment: evidence for an ,activity- stat' in young children (EarlyBird 16). International Journal of Obesity, 30, 1050-1055.

Wilkinson, K.M. (2008). Increasing obesity in children and adolescents: an alarming epidemic. JAAPA, 21, 31-36, 38. 
Will, N., Schmidt, S., \& Woll, A. (2016). Intensität und soziale Disparität sportlicher Aktivität in Schule und Verein. Die Motorik-Modul-Studie (MoMo). Sportunterricht, 65(8), 239244.

Williams, H.G., Pfeiffer, K.A., O‘neill, J.R., Dowda, M., Mci- ver, K.L., Brown, W.H. \& Pate, R.R. (2008). Motor skill performance and physical activity in preschool children. Obesity (Silver Spring), 16, 1421-1426.

Woll, A. (1996). Gesundheitsförderung in der Gemeinde. Neu- Isenburg.

Woll, A. \& Bös, K. (2004). Wirkungen von Gesundheitssport. Bewegungstherapie \& Gesundheitssport 20:1-10.

Zeiher, H. (1983). Die vielen Räume der Kinder. Zum Wandel räumlicher Lebensbedingungen seit 1945. In: Preuss-Lausitz et al. (Hrsg.), S. 176-195. Weinheim: Beltz.

Züchner, I. (2010). Operation Ganztagsschule. DJI Bulettin, 91 (3), 4-7.

\section{Eidesstattliche Erklärung}

Ich erkläre, dass ich die Arbeit selbständig angefertigt und nur die angegebenen Hilfsmittel benutzt habe. Alle Stellen, die dem Wortlaut oder dem Sinn nach anderen Werken, gegebenenfalls auch elektronischen Medien, entnommen sind, sind von mir durch Angabe der Quelle und des Zugriffsdatums sowie dem Ausdruck der ersten Seite belegt; sie liegen zudem für den Zeitraum von 2 Jahren entweder auf einem elektronischen Speichermedium im PDF-Format oder in gedruckter Form vor.

Freiburg, den 21.08.2017 


\section{About the author:}

Christopher Winterhalder studied German and Physical Education for das Lehramt an Gymnasien, at the Albert-Ludwigs-Universität- Freiburg. He is married and has four kids. Besides spending time with his family, he loves reading and different sports e.g. soccer, beachvolleyball, squash and Brazilian Jiu-Jitsu.

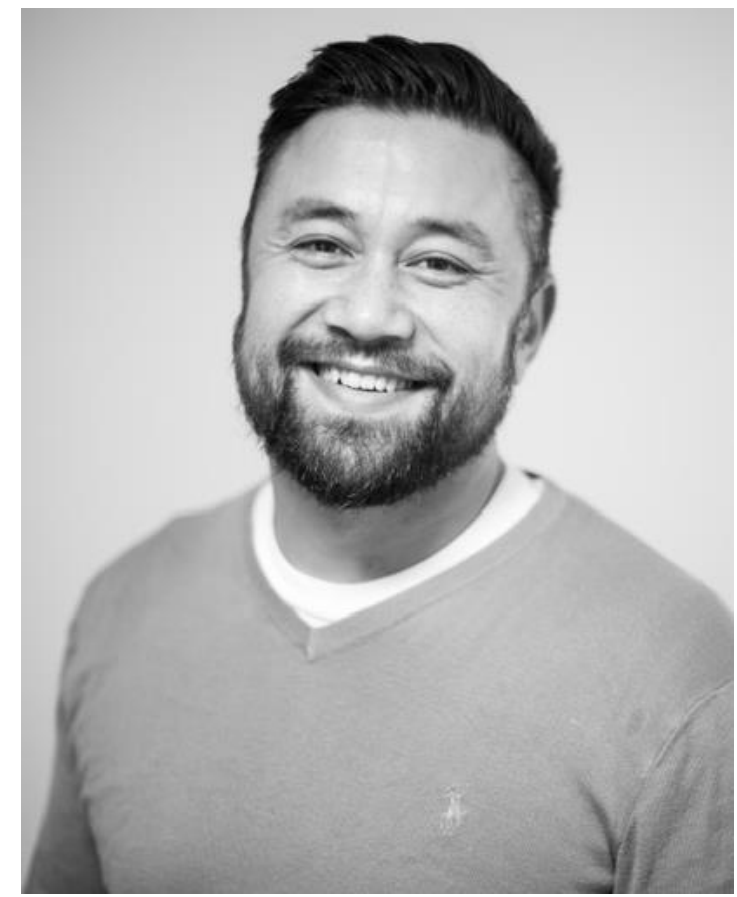

